Part of Journal of Research of the National Bureau of Standards, Volume 25, December 1940

\title{
MUTUAL INDUCTANCE OF TWO HELICES WHOSE AXES ARE PARALLEL
}

\author{
By Chester Snow
}

\begin{abstract}
Formulas are obtained for the mutual inductance of two helical wires whose axes are parallel. The formulas are given as series in oblate spheroidal harmonics. The principal part is the inductance of the two corresponding current sheets, the correction terms being small when the coils are closely wound, so that the pitch is small compared to the diameter of the cylindrical form in each case.
\end{abstract}

\section{CONTENTS}

I. Introduction

II. Definitions and integral formulas for the mutual inductance of the two helices and of their current sheets _ _

III. Difference between the formula for the mutual inductance of two helices and that of their current sheets _.

IV. Expansion of $\omega$ in a double Fourier's series of the azimuthal angles -634

V. Partial differential equations and boundary conditions satisfied by the function $\varphi_{n_{1}, n_{2}}$

VI. Expansion of $\varphi_{n_{1}, n_{2}}$ in oblate spheroidal coordinates _...

VII. Expansion of $\omega_{n_{1}, n_{2}}$ in spheroidal coordinates _... 658

VIII. Corrections for diameter of the wires_.

IX. Summary

\section{INTRODUCTION}

The principal part of the mutual inductance between two helices whose axes are parallel is that between the two associated current sheets. This approximation may not be sufficient when the inductor is used for absolute electrical measurements of high precision. In this paper formulas are obtained which, in addition to the principal term, contain correction terms of the first and second order in the small quantities, such as the ratio of the pitch of the windings to their cylindrical radii.

The electromagnetic force between currents in the helices may be obtained by differentiation with respect to their relative coordinates. Such helices are not used as often as coaxial ones, although they do occur, for example, in the Ayrton-Jones type of current balance as used by the English National Physical Laboratory.

\section{DEFINITIONS AND INTEGRAL FORMULAS FOR THE MUTUAL INDUCTANCE OF TWO HELICES AND OF THEIR CURRENT SHEETS}

The two helices are shown in figure 1 . The axis of the first, $h_{1}$, is the $x$-axis; that of the second, $h_{2}$, is the line in the $x y$ plane parallel to the $x$-axis at a distance $r$ from it, where $r \geqq 0$. If $r=0$, the helices are coaxial. 
The cylindrical radius of the first is $a_{1}$; its pitch, $2 \pi p_{1}$; and its axial length, $l_{1}=2 \pi p_{1} N_{1}$, where $N_{1}$, the "total number of turns," is not restricted to be an integer. The "number of turns per unit axia, length" is $n_{1}=1 / 2 \pi p_{1}$, not necessarily an integer. The initial planel

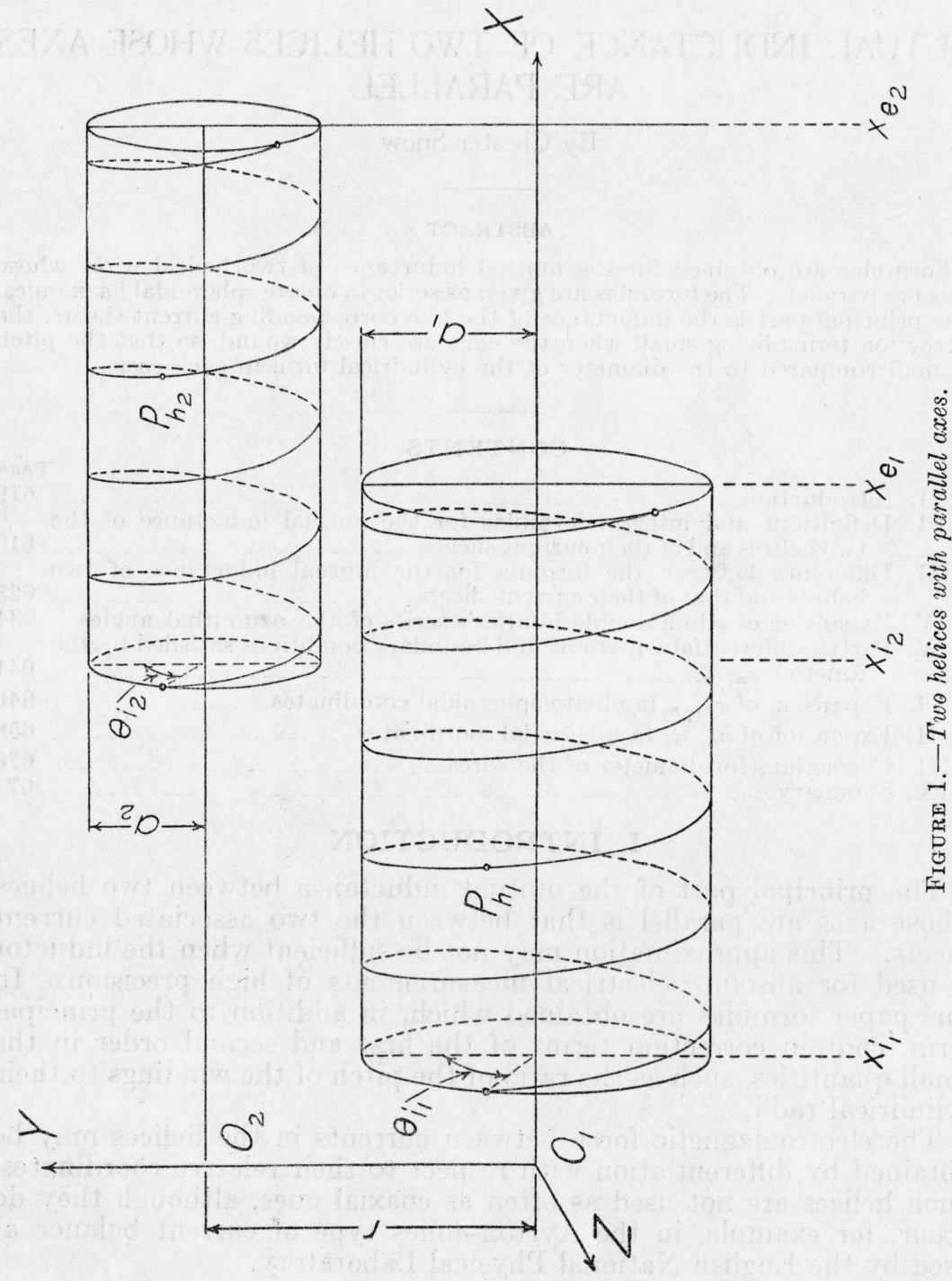

$x=x_{i_{1}}$, contains its initial point, $P_{i_{1}}$, and the end plane, $x=x_{e_{1}}$ its end point, $P_{e_{1}}$, so that $l_{1}=2 \pi p_{1} N_{1}=x_{e_{1}}-x_{i_{1}}>0$. The current sheet, $S_{1}$, "associated with the helix, $h_{1}$," is the cylindrical surface (generated by rotating the helix about its axis through an angle $2 \pi$ ), upon which there is a surface current of unit strength (in electromagnetic cgs units), uniformly distributed, the direction of the current at any point being that of the helical filament passing through that point. This is equivalent to defining the current sheet as that part, included between 
the terminal planes $x=x_{i}$ and $x=x_{e}$, of an endless cylinder which is completely covered by a helical tape winding of axial breadth equal to the pitch, so that no insulating space is left between successive windings. The angular current is $n_{1}=1 / 2 \pi p_{1}$ per unit axial length. The axial current is $1 / 2 \pi a_{1}$ per unit length along a circular arc for which $x$ is constant.

If subscripts 2 refers to the second helix, $h_{2}$, or its sheet $S_{2}$, it follows from this definition that the mutual inductance, $M_{0}$, between the two current sheets is given by the double surface integral taken over the two sheets.

$$
\begin{aligned}
M_{0} & =\frac{1}{4 \pi^{2} p_{1} p_{2}} \iint d S_{1} \iint F\left(x_{2}-x_{1}, \theta_{1}, \theta_{2}\right) d S_{2} \\
& =\frac{a_{1} a_{2}}{4 \pi^{2} p_{1} p_{2}} \int_{0}^{2 \pi} d \theta_{1} \int_{x_{i_{1}}}^{x_{\varepsilon_{1}}} d x_{1} \int_{0}^{2 \pi} d \theta_{2} \int_{x_{i_{2}}}^{x_{\iota_{2}}} d x_{2} F\left(x_{2}-x_{1}, \theta_{1}, \theta_{2}\right),
\end{aligned}
$$

where

$$
F\left(x_{2}-x_{1}, \theta_{1}, \theta_{2}\right) \equiv \frac{\cos \left(\theta_{2}-\theta_{1}\right)+p_{1} p_{2} / a_{1} a_{2}}{R_{12}\left(x_{2}-x_{1}, \theta_{1}, \theta_{2}\right)},
$$

and $R_{12}$ is the distance from a point $P_{1}\left(x_{1}, y_{1}, z_{1}\right)$ on $S_{1}$ to $P_{2}\left(x_{2}, y_{2}, z_{2}\right)$ on $S_{2}$, so that

$$
\begin{aligned}
R_{2}\left(x_{2}-x_{1}, \theta_{1}, \theta_{2}\right)=\left(x_{2}-x_{1}\right)^{2}+a_{1}^{2}+a_{2}^{2}-2 a_{1} a_{2} \cos \left(\theta_{2}-\theta_{1}\right) & \\
& \left.+r^{2}+2 r\left(a_{2} \cos \theta_{2}-a_{1} \cos \theta_{1}\right) .\right]
\end{aligned}
$$

The name "mutual inductance" is used here for the Neumann integral, so that when it refers to an unclosed circuit the implication is that the mutual inductance of the part (lead wires, etc.) which must be added to close the circuit must be evaluated by a Neumann's integral. With this understanding, it is evident that the term $p_{1} p_{2} / a_{1} a_{2}$ in the numerator of eq 2 gives rise in eq 1 to the mutual inductance of the axial currents in the two sheets, whereas the term " $\cos \left(\theta_{2}-\theta_{1}\right)$ " gives rise to that due to their angular currents only.

In the integral (eq 1) the two independent variables, $x_{1}, \theta_{1}$, are cylindrical coordinates referred to the axes of the first cylinder, and $x_{2}, \theta_{2}$ are cylindrical coordinates referred to the axes of the second cylinder, so that the angles $\theta_{1}$ and $\theta_{2}$ each range over the interval from zero to $2 \pi$. If $x_{1}, y_{1}, z_{1}$ are the rectangular coordinates of any point $P_{1}$ on $S_{1}$, the position of $P_{1}$ may be specified by assigning the pair of cylindrical coordinates $x_{1}$ and $\theta_{1}$ (with cylindrical radius $a_{1}$ ), or it may be specified by assigning the pair of semihelical coordinates $\theta_{i_{1}}, \theta_{1}$, the equations of transformation being

$$
\left\{\begin{array}{l}
x_{1}=x_{i_{1}}+p_{1}\left(\theta_{1}-\theta_{i}\right) \\
y_{1}=a_{1} \cos \theta_{1} \\
z_{1}=a_{1} \sin \theta_{1}
\end{array}\right\} \text {, where }\left\{\begin{array}{c}
0 \leqslant \theta_{i_{1}}<2 \pi \\
\theta_{i_{1}} \leqslant \theta_{1} \leqslant \theta_{e_{1}} \equiv \theta_{i_{1}}+2 \pi N_{1}
\end{array}\right\} \text {. }
$$

Considering the pair of variables $\theta_{1}$ and $\theta_{i_{1}}$ for this range as two independent parameters which suffice to determine the position of $P_{1}$ on $S_{1}$, eq 4 are the parametric equations of the cylindrical surface $S_{1}$. The locus of the equation $\theta_{i_{1}}=$ constant, is a helix, $h_{1}$, of pitch $2 \pi p_{1}$ lying on $S_{1}$, while the locus of the equation $\theta_{1}=$ constant is a straight line of length $2 \pi p_{1}$ (or less) lying on $S_{1}$. 
When the point $\mathrm{P}_{1}\left(x_{1} y_{1} z_{1}\right)$ is also on the helix $h_{1}$, this may be designated by $P_{h_{1}}\left(x_{h_{1}}, y_{h_{1}}, z_{h_{1}}\right)$, the $x_{h_{1}}, y_{h_{1}}$, and $z_{h_{1}}$ being given as in eq 4 with the understanding that $\theta_{i_{1}}$ is a given constant and not a variable parameter.

The equations

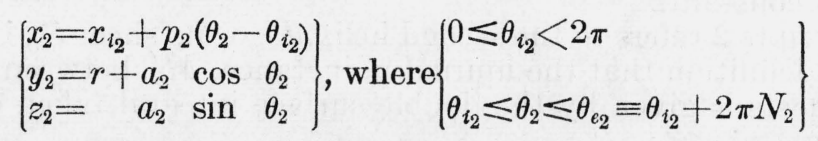

are the parametric equations of the sheet $S_{2}$ when $\theta_{2}$ and $\theta_{i_{2}}$ are both variables. The locus of the equation $\theta_{i_{2}}=$ constant is a helix $h_{2}$ of pitch $2 \pi p_{2}$ lying on $S_{2}$.

The mutual inductance (Neumann's integral) of the helices, $h_{1}$ and $h_{2}$, is

$$
M=\int_{0}^{s_{1}} d s_{1} \int_{0}^{s_{2}} d s_{2} \frac{\cos \left(d s_{1}, d s_{2}\right)}{R_{12}}
$$

taken along both helices, where $s_{1}$ and $s_{2}$ denote total length of arc.

From eq 4 it is found that the element of length $d s_{1}$ has the magnitude

$$
d s_{1}=\sqrt{\left(a_{1}^{2}+p_{1}^{2}\right)} d \theta_{1}
$$

and the direction cosines

$$
\frac{d x_{1}}{d s_{1}}=\frac{p_{1}}{\sqrt{a_{1}^{2}+p_{1}^{2}}}, \frac{d y_{1}}{d s_{1}}=-\frac{a_{1} \sin \theta_{1}}{\sqrt{a_{1}^{2}+p_{1}^{2}}}, \frac{d z_{1}}{d s_{1}}=\frac{a_{1} \cos \theta_{1}}{\sqrt{a_{1}^{2}+p_{1}^{2}}} .
$$

The total length of the helix is $s_{1}$, where

$$
s_{1}=2 \pi N_{1} \sqrt{a_{1}^{2}+p_{1}^{2}}=\frac{l_{1}}{p_{1}} \sqrt{a_{1}^{2}+p_{1}^{2}},
$$

where $l_{1}$ is its "axial length."

The same formulas, with change of subscript, refer to $h_{2}$, so that

$$
d s_{1}, d s_{2} \cos \left(d s_{1}, d s_{2}\right)=a_{1} a_{2}\left[\cos \left(\theta_{2}-\theta_{1}\right)+\frac{p_{1} p_{2}}{a_{1} a_{2}}\right] d \theta_{1} d \theta_{2} .
$$

Hence the mutual inductance of the two helices is

$$
\begin{aligned}
M & =a_{1} a_{2} \int_{\theta_{i_{1}}}^{\theta_{\theta_{1}}} d \theta_{1} \int_{\theta_{i_{2}}}^{\theta_{\theta_{2}}} d \theta_{2} \frac{\cos \left(\theta_{2}-\theta_{1}\right)+p_{1} p_{2} / a_{1} a_{2}}{R_{12}\left(x_{h_{2}}-x_{h_{1}}, \theta_{1}, \theta_{2}\right)}= \\
& =a_{1} a_{2} \int_{\theta_{i_{1}}}^{\theta_{\theta_{1}}} d \theta_{1} \int_{2}^{\theta_{\theta_{2}}} d \theta_{2} F\left(x_{h_{2}}-x_{h_{1}}, \theta_{1}, \theta_{2}\right),
\end{aligned}
$$

where $x_{h_{1}}$ is a function of $\theta_{1}$ only and $x_{h_{2}}$ of $\theta_{2}$ only, given by eq 4 and 5 , the $\theta_{i_{1}}$ and $\theta_{i_{2}}$ being constants.

In the general case of helices whose axes are merely parallel, not necessarily coaxial, and whose terminal planes are arbitrary, eq 1 for the current sheets is sufficiently complicated, but eq 9 for the helices is even more troublesome. So far as we are aware, neither of these integrals may be evaluated in finite terms in general, although the 
former may (in the case of coaxial current sheets) be so evaluated in terms of the three complete elliptic integrals. This is Jones' formula. ${ }^{1}$

The present paper is limited to what may, for brevity, be called the practical case, in which the two current sheets do not cut or touch each other, in fact they do not lie at any points close to each other. More precisely, considering the pitches $2 \pi p_{1}$ and $2 \pi p_{2}$ as infinitesimals of the first order compared to finite lengths like $a_{1}, a_{2}$, then the distance, $R_{12}$ of a point $P_{1}$ on $S_{1}$ from a point $P_{2}$ on $S_{2}$, is, for all points, $P_{1}$ and $P_{2}$, finite (not a small quantity of the order of the pitch). The various expansions in infinite series to be obtained below for the current sheet are not subject to this limitation but cover the "impractical" cases also. The restriction is necessary, however, in order to obtain a practicable quantitative expression for the relatively small difference between the mutual inductance of the two helices and that of their associated current sheets.

If $f(x, \theta)$ is a continuous and single-valued function of position on a cylindrical sheet $S$, it must be a periodic function of $\theta$ with period $2 \pi$, and the line average of $f$ along a helix on $S$ is obviously very nearly the same as its surface average over $S$, provided that the pitch is small.

In the following section the two are compared, and an expression for their difference is obtained to the second order of small quantities inclusive, and application is made to the problem in hand, because, with closely wound coils, the precision of electrical measurements at present attainable requires formulas valid to the second order.

The general case of coaxial helices has been treated in an earlier paper, ${ }^{2}$ so the emphasis here is upon the noncoaxial case.

\section{DIFFERENCE BETWEEN THE FORMULA FOR THE MUTUAL INDUCTANCE OF TWO HELICES AND THAT OF THEIR CURRENT SHEETS}

Certain problems relating to the helix could be treated more elegantly by the use of semihelical coordinates or by orthogonal helical ones. A plane rectangle ruled with two mutually orthogonal families of straight lines could be wrapped around a cylindrical surface, and the parameters defining these families would be orthogonal helical coordinates. However, it has been found by trial that the comparison of a line integral along a helix with a surface integral over its sheet may be made most simply by the use of ordinary cylindrical coordinates $(x, \theta)$ because the $x$ limits and $\theta$ limits of the surface integral are independent of each other.

As the preliminary formulation applies to either helix $h_{1}$ or $h_{2}$, the subscripts may be omitted. In figure 2 , with $x$ as abscissa and $a \theta$ as ordinate, the rectangle represents the development of the cylindrical sheet $S$. The helix $h$ is the system of parallel lines whose separation in the $x$-direction is $\Delta x \equiv 2 \pi p=$ the pitch of the helix. The slope of these straight lines in figure 2 is $p / a$.

The variable $\theta$ lies between zero and $2 \pi$, and the initial azimuth, $\theta_{i}$, also lies in that interval, but the end azimuth, $\theta_{e}$, as defined in eq 4 or 5 , does not, since $\theta_{e}=\theta_{i}+2 \pi N$. We, therefore, (for the moment) dis-

I J. V. Jones, Proc. Roy. Soc. (London) [A] 63, 198 (1898).

2 Chester Snow, Mutual inductance and force between two coaxial helical wires, J. Research NBS 22, 269 (1939) RP 1178. 
tinguish between $\theta_{e}$ and $\bar{\theta}_{e}$, where $\bar{\theta}_{e} \equiv \theta_{e}-2 \pi N^{\prime}=\theta_{i}+2 \pi \quad\left(N-N^{\prime}\right)$, where $N^{\prime}$ is the largest positive integer which is less than or equal to $\theta_{e} / 2 \pi$. When $\bar{\theta}_{e}$ is thus chosen, it lies in the interval, $0 \leqslant \bar{\theta}_{e} \leqslant 2 \pi$, as

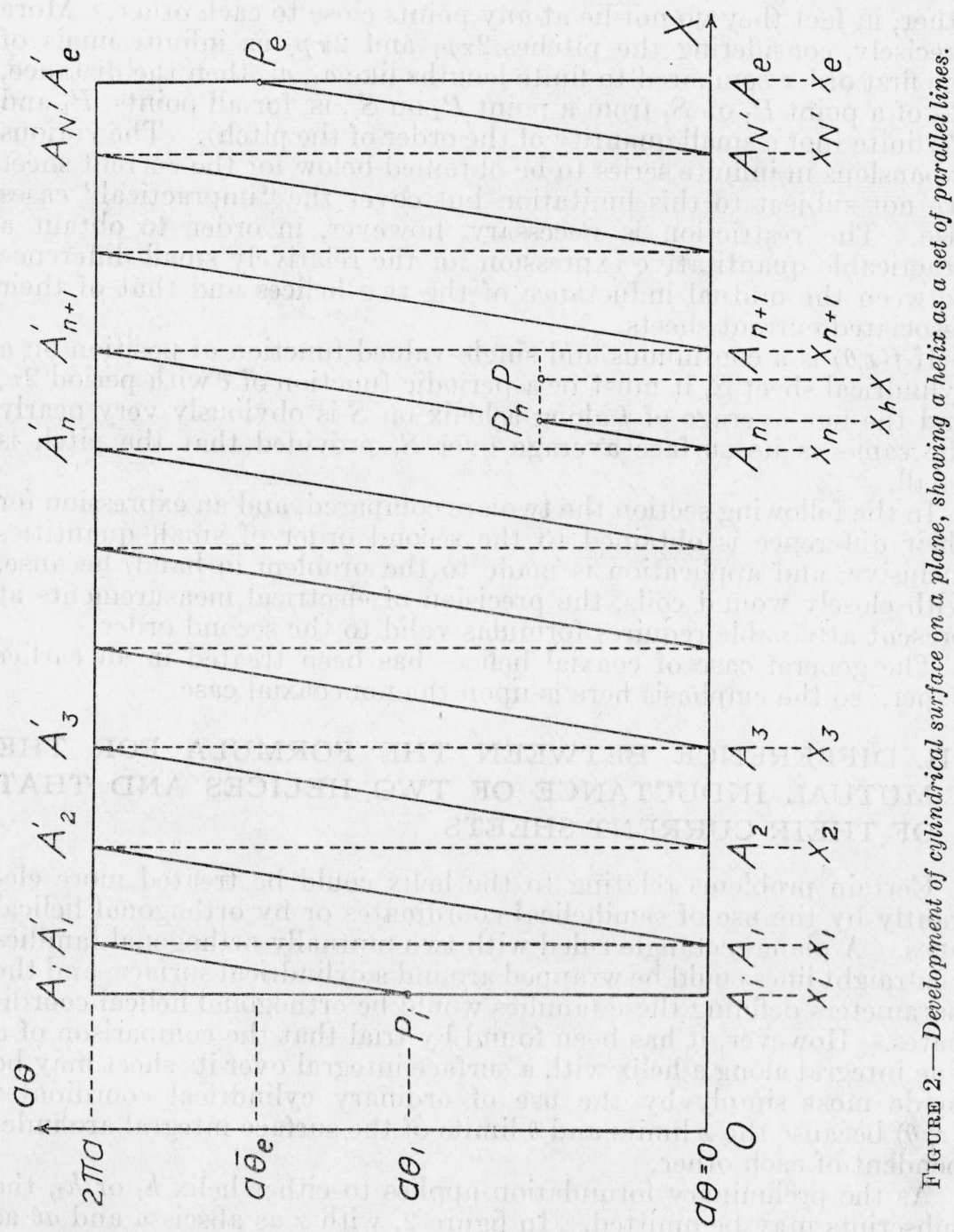

indicated in figure 2. The points $A_{1}, A_{2}, \ldots A_{N^{\prime}}$ have abscissa $x_{n}$, where

$$
x_{n}=x_{i}-p \theta_{i}+n \Delta x \text { for } n=1,2,3, \ldots N^{\prime} \text {. }
$$

Hence the equation of the helix for figure 2 is

$x_{h}=x_{i}+p\left(\theta-\theta_{i}\right)$ when $x_{i} \leqslant x<x_{1}$ and $\theta_{i} \leqslant \theta<2 \pi$

$=x_{n}+p \theta \quad$ when $x_{n} \leqslant x<x_{n+1}$ and $0 \leqslant \theta<2 \pi$ for $n=1,2,3, \ldots N^{\prime}-1$ $=x_{e}+p\left(\theta-\bar{\theta}_{e}\right)$ when $x_{N^{\prime}} \leqslant x<x_{e}$ and $0 \leqslant \theta<\bar{\theta}_{e}$. 
Let $f(x, \theta)$ be a finite, continuous, and single-valued function of position on $S$. It is, therefore a periodic function of its second argument $\theta$, with period $2 \pi$. The same character is assumed for $f^{\prime}(x, \theta)$ and $f^{\prime \prime}(x, \theta)$, where primes denote partial derivatives with respect to the $x$-argument.

Hence, by eq 6 , the line integral along a helix of $f(x, \theta)\left(a^{2}+p^{2}\right)^{-\frac{1}{2}}$ is

$$
\begin{gathered}
\sqrt{\frac{1}{a_{2}+p_{2}}} \int_{0}^{s} f(x, \theta) d s=\int_{\theta_{i}}^{\theta_{e}} f\left(x_{h}, \theta\right) d \theta= \\
=\int_{\theta_{i}}^{2 \pi} f\left(x_{i}+p\left[\theta-\theta_{i}\right], \theta\right) d \theta+\int_{0}^{2 \pi} d \theta \sum_{n=1}^{N^{\prime}-1} f\left(x_{n}+p \theta, \theta\right) \\
+\int_{0}^{\vec{\theta}_{e}} f\left(x_{e}+p\left[\theta-\theta_{e}\right], \theta\right) d \theta .
\end{gathered}
$$

The terms in the summation represent the principal part, the first and last integrals being relatively small (of the first order). Hence in them the integrand may be expanded by Taylor's theorem as a function of its $x$-argument, about the terminal values of $x$, so that

$$
\begin{aligned}
& \frac{a}{\sqrt{a^{2}+p^{2}}} \int_{0}^{s} f(x, \theta) d s=a \int_{0}^{2 \pi} d \theta \sum_{n=1}^{N^{\prime}-1} f\left(x_{n}+p \theta, \theta\right) \\
& +a \int_{0}^{\theta_{\theta}} d \theta\left[f\left(x_{e}, \theta\right)+p\left(\theta-\bar{\theta}_{e}\right) f^{\prime}\left(x_{e}, \theta\right)\right] \\
& +a \int_{\theta_{i}}^{2 \pi} d \theta\left[f\left(x_{i}, \theta\right)+p\left(\theta-\theta_{i}\right) f^{\prime}\left(x_{i}, \theta\right)\right] .
\end{aligned}
$$

To transform the summation, consider the following integral taken with respect to $x$, holding $\theta$ constant,

$$
\int_{x 1}^{x_{N^{\prime}}} f(x, \theta) d x=\sum_{n=1}^{N^{\prime}-1} \int_{x_{n}}^{x_{n}+\Delta x} f(x, \theta) d x,
$$

where the integral is taken along the horizontal dotted line passing through $P_{h}$ and $P$ in figure 2, the latter point $P$ being the general point of integration with abscissa $x=x_{h}+\left(x-x_{h}\right)$, so that expanding $f$ as a function of $x$ (with $\theta$ constant) about the point $P_{h}$ on the helix in this $n^{h}$ strip, we find

$$
\begin{aligned}
\int_{x_{n}}^{x_{n}+\Delta x} f(x, \theta) d x & =\int_{x_{n}}^{x_{n}+\Delta x} f\left(x_{h}+\left[x-x_{h}\right], \theta\right) d x \\
& =\int_{x_{n}}^{x_{n}+2 \pi p} d x\left[f\left(x_{h}, \theta\right)+\left(x-x_{h}\right) f^{\prime}\left(x_{h}, \theta\right)+\frac{\left(x-x_{h}\right)^{2}}{2} f^{\prime \prime}\left(x_{h}, \theta\right)\right] .
\end{aligned}
$$

On performing the integration with respect to $x$ ( $\theta$-and $x_{h}$ being constant), these become, after placing $x_{h}=x_{n}+p \theta$,

$$
\begin{aligned}
& \int_{x_{n}}^{x_{n}+\Delta x} f(x, \theta) d x= \\
& =2 \pi p\left\{f\left(x_{n}+p \theta, \theta\right)+p(\pi-\theta) f^{\prime}\left(x_{n}+p \theta, \theta\right)+\frac{p^{2}}{2}\left[(\pi-\theta)^{2}+\frac{\pi^{2}}{3}\right] f^{\prime \prime}\left(x_{n}+p \theta, \theta\right)\right\},
\end{aligned}
$$


so that eq 11 becomes

$$
\begin{aligned}
& \int_{x_{1}}^{x_{N^{\prime}}} f(x, \theta) d x= \\
& =2 \pi p \sum_{n=1}^{N^{\prime}-1} \\
& \left\{f\left(x_{n}+p \theta, \theta\right)+p(\pi-\theta) f^{\prime}\left(x_{n}+p \theta, \theta\right)+\frac{p^{2}}{2}\left[(\pi-\theta)^{2}+\frac{\pi^{2}}{3}\right] f^{\prime \prime}\left(x_{n}+p \theta, \theta\right)\right\} .
\end{aligned}
$$

Applying the same formula to $f^{\prime}(x, \theta)$ gives, after multiplying by $p(\pi-\theta)$,

$$
\begin{aligned}
& p(\pi-\theta) \int_{x_{1}}^{x_{N^{\prime}}} f^{\prime}(x, \theta) d x= \\
& \quad=2 \pi p \sum_{-n=1}^{N^{\prime}-1}\left\{p(\pi-\theta) f^{\prime}\left(x_{n}+p \theta, \theta\right)+p^{2}(\pi-\theta)^{2} f^{\prime \prime}\left(x_{n}+p \theta, \theta\right)\right\},
\end{aligned}
$$

which carries the approximation to the same order as in eq 12. Also, by eq 12 , we may write to the same approximation

$$
p^{2} \int_{x_{1}}^{x_{N^{\prime}}^{\prime \prime}} f^{\prime \prime}(x, \theta) d x=2 \pi p \sum_{n=1}^{N^{\prime}-1} p^{2} f\left(x_{n}+p \theta, \theta\right) .
$$

Subtracting eq $12^{\prime}$ from eq 12 , and making use of eq $12^{\prime \prime}$, gives

$$
\begin{aligned}
\sum_{n=1}^{N^{\prime}-1} f\left(x_{n}+p \theta, \theta\right) & = \\
= & \int_{x_{1}}^{x_{N}} d x\left\{\frac{(f x, \theta)}{2 \pi p}+\left(\frac{\theta-\pi}{2 \pi}\right) f^{\prime}(x, \theta)+\frac{p}{4 \pi}\left[(\theta-\pi)^{2}-\frac{\pi^{2}}{3}\right] f^{\prime \prime}(x, \theta)\right\}
\end{aligned}
$$

On integrating the second and third terms, this becomes

$$
\begin{aligned}
\sum_{n=1}^{N^{\prime}-1} f\left(x_{n}+p \theta ; \theta\right) & =\frac{1}{2 \pi p} \int_{x_{i}}^{x} f(x, \theta) d x-\frac{1}{2 \pi p}\left[\int_{x_{i}}^{x_{1}} f(x, \theta) d x+\int_{x_{N^{\prime}}}^{x_{x}} f(x, \theta) d x\right] \\
& +\left(\frac{\theta-\pi}{2 \pi}\right)\left[f\left(x_{N^{\prime}}, \theta\right)-f\left(x_{1}, \theta\right)\right] \\
& +\frac{p}{4 \pi}\left[\left(\theta-\pi^{2}-\frac{\pi^{2}}{3}\right]\left[f^{\prime}\left(x_{N^{\prime}}, \theta\right)-f^{\prime}\left(x_{1}, \theta\right)\right] .\right.
\end{aligned}
$$

In the terms of smallest order we may place $f^{\prime}\left(x_{N^{\prime}}, \theta\right)=f^{\prime}\left(x_{e}, \theta\right)$ and $f^{\prime}\left(x_{1}, \theta\right)=f^{\prime}\left(x_{i}, \theta\right)$. In the terms involving $f\left(x_{N^{\prime}}, \theta\right)$ and $f\left(x_{1}, \theta\right)$, which relative to the principal term, are of first order, we may place

Also

$$
\begin{gathered}
f\left(x_{N^{\prime}}, \theta\right)=f\left(x_{e}-p \bar{\theta}_{e}, \theta\right)=f\left(x_{e}, \theta\right)-p \bar{\theta}_{e} f^{\prime}\left(x_{e}, \theta\right) \\
f\left(x_{1}, \theta\right)=f\left(x_{i}+p\left[2 \pi-\theta_{i}\right], \theta\right)=f\left(x_{i}, \theta\right)+p\left(2 \pi-\theta_{i}\right) f^{\prime}\left(x_{i}, \theta\right) .
\end{gathered}
$$

$$
\begin{aligned}
\frac{-1}{2 \pi p} \int_{x_{i}}^{x_{1}} f(x, \theta) d x & =\frac{-1}{2 \pi p} \int_{x_{i}}^{x_{i}+p\left(2 \pi-\theta_{i_{1}}\right)} d x\left\{f\left(x_{i}, \theta\right)+\left(x-x_{i}\right) f^{\prime}\left(x_{i}, \theta\right)\right\} \\
& =-\left(\frac{2 \pi-\theta_{i}}{2 \pi}\right)\left[f\left(x_{i}, \theta\right)+\frac{p}{2}\left(2 \pi-\theta_{i}\right) f^{\prime}\left(x_{i}, \theta\right)\right] .
\end{aligned}
$$


Similarly,

$$
\begin{aligned}
\frac{-1}{2 \pi p} \int_{x_{N^{\prime}}}^{x_{e}} f(x, \theta) d x & =\frac{-1}{2 \pi p} \int_{x_{e}-\bar{\theta}_{e}}^{x_{\theta}} d x\left\{f\left(x_{e}, \theta\right)+\left(x-x_{e}\right) f^{\prime}\left(x_{e}, \theta\right)\right\} \\
& =-\frac{\bar{\theta} e}{2 \pi}\left[f\left(x_{e}, \theta\right)-\frac{p \bar{\theta}_{e}}{2} f^{\prime}\left(x_{e}, \theta\right)\right] .
\end{aligned}
$$

By use of these expansions, eq 13 becomes

$$
\begin{gathered}
\sum_{n=1}^{N^{\prime}-1} f\left(x_{n}+p \theta, \theta\right)=\frac{1}{2 \pi p} \int_{x_{i}}^{x_{e}} f(x, \theta) d x \\
+\left(\frac{\theta-\bar{\theta}_{e}-\pi}{2 \pi}\right) f\left(x_{e}, \theta\right)+\frac{p}{\pi}\left(\left[\frac{\theta-\bar{\theta}_{e}-\pi}{2}\right)^{2}-\frac{\pi^{2}}{12}\right] f^{\prime}\left(x_{e}, \theta\right) \\
-\left(\frac{\theta-\theta_{i}+\pi}{2 \pi}\right) f\left(x_{i}, \theta\right)-\frac{p}{\pi}\left[\left(\frac{\theta-\theta_{i}+\pi}{2}\right)^{2}-\frac{\pi^{2}}{12}\right] f^{\prime}\left(x_{i}, \theta\right) .
\end{gathered}
$$

The series on the right side of eq 10 is found by multiplying this by $a d \theta$ and integrating from $\theta=0$ to $\theta=2 \pi$. Equation 10 then becomes

$$
\begin{gathered}
\frac{a}{\sqrt{a^{2}+p^{2}}} \int_{0}^{s} f(x, \theta) d s=\frac{a}{2 \pi p} \int_{0}^{2 \pi} d \theta \int_{x_{i}}^{x_{e}} d x f(x, \theta) \\
+\frac{a}{\pi} \int_{0}^{\bar{\theta}_{e}} d \theta\left\{\left[\frac{\pi-\left(\bar{\theta}_{e}-\theta\right)}{2}\right] f\left(x_{e}, \theta\right)+p\left(\left[\frac{\pi-\left(\bar{\theta}_{e}-\theta\right)}{2}\right]^{2}-\frac{\pi^{2}}{12}\right) f^{\prime}\left(x_{e}, \theta\right)\right\} \\
+\frac{a}{\pi} \int_{\bar{\theta}_{e}}^{2 \pi} d \theta\left\{\left[\frac{\theta-\bar{\theta}_{e}-\pi}{2}\right] f\left(x_{e}, \theta\right)+p\left(\left[\frac{\theta-\bar{\theta}_{e}-\pi}{2}\right]-\frac{\pi^{2}}{12}\right) f^{\prime}\left(x_{e}, \theta\right)\right\} \\
-\frac{a}{\pi} \int_{0}^{\theta_{i}} d \theta\left\{\left[\frac{\pi-\left(\theta_{i}-\theta\right)}{2}\right] f\left(x_{i}, \theta\right)+p\left(\left[\frac{\pi-\left(\theta_{i}-\theta\right)}{2}\right]^{2}-\frac{\pi^{2}}{12}\right) f^{\prime}\left(x_{i}, \theta\right)\right\} \\
-\frac{a}{\pi} \int_{\theta_{i}}^{2 \pi} d \theta\left\{\left[\frac{\theta-\theta_{i}-\pi}{2}\right] f\left(x_{i}, \theta\right)+p\left(\left[\frac{\theta-\theta_{i}-\pi}{2}\right]^{2}-\frac{\pi^{2}}{12}\right) f\left(x_{i}, \theta\right)\right\}
\end{gathered}
$$

To reduce eq 14 to a more convenient form, consider the function $\theta\left(\theta_{i}-\theta\right)$, which is an odd periodic function of its argument with period $2 \pi$, having a finite discontinuity when its argument is zero or $2 \pi n$. It is defined for all other values of its argument by the series

so that

$$
\theta\left(\theta_{i}-\theta\right)=\sum_{n=1}^{\infty} \frac{\sin n\left(\theta_{i}-\theta\right)}{n},
$$

$$
\left.\begin{array}{rl}
\theta\left(\theta_{i}-\theta\right) & =\frac{\pi}{2}-\left(\frac{\theta_{i}-\theta}{2}\right), \text { when } 0<\theta_{i}-\theta<2 \pi \\
& =-\frac{\pi}{2}-\left(\frac{\theta_{i}-\theta}{2}\right), \text { when }-2 \pi<\theta_{i}-\theta<0 .
\end{array}\right\}
$$


Applying the theorem (eq 16) to eq 17 gives

$$
\begin{gathered}
M=\frac{1}{2 \pi p_{2}}\left(\iint_{s_{2}} d S_{2} f\left(x_{2}, \theta_{2}\right)\right. \\
+2 a_{2} \int_{0}^{2 \pi} d \theta_{2}\left\{p_{2} \Theta\left(\theta_{e_{2}}-\theta_{2}\right) f\left(x_{e_{2}}, \theta_{2}\right)+\left[p_{2}^{2} \theta^{2}\left(\theta_{e_{2}}-\theta_{2}\right)-\frac{\pi^{2} p_{2}^{2}}{12}\right] f^{\prime}\left(x_{e_{2}}, \theta_{2}\right)\right\} \\
-2 a_{2} \int_{0}^{2 \pi} d \theta_{2}\left\{p_{2} \Theta\left(\theta_{i_{2}}-\theta_{2}\right) f\left(x_{i_{2}}, \theta_{2}\right)+\left[p_{2}^{2} \theta^{2}\left(\theta_{i_{2}}-\theta_{2}\right)-\frac{\pi^{2} p_{2}^{2}}{12}\right] f^{\prime}\left(x_{i_{2}}, \theta_{2}\right)\right\}
\end{gathered}
$$

We may now introduce into the various integrals of eq 19 the expressions for $f\left(x_{2}, \theta_{2}\right), f\left(x_{e_{2}}, \theta_{2}\right), f\left(x_{i_{2}}, \theta_{2}\right), f^{\prime}\left(x_{e_{2}}, \theta_{2}\right)$, and $f^{\prime}\left(x_{i_{2}}, \theta_{2}\right)$ obtained from eq 18. We retain only three orders of magnitude.

Since $f^{\prime}$ occurs only in the second-order terms of eq 19, we need only the principal part of $\mathrm{f}^{\prime}$, which is the $x_{2}$ - derivative of the principal term in eq 18 so that we place

$$
\begin{aligned}
{ }^{\prime}\left(x_{2}, \theta_{2}\right) & =\frac{a_{1}}{2 \pi p_{1}} \int_{0}^{2 \pi} d \theta_{1} \int_{x_{i_{1}}}^{x_{\theta_{1}}} d x_{1} F^{\prime}\left(x_{2}-x_{1}, \theta_{1}, \theta_{2}\right) \\
& =\frac{-a_{1}}{2 \pi p_{1}} \int_{0}^{2 \pi} d \theta_{1}\left\{F\left(x_{2}-x_{e_{1}}, \theta_{1}, \theta_{2}\right)-F\left(x_{2}-x_{i_{1}}, \theta_{1}, \theta_{2}\right)\right\} .
\end{aligned}
$$

This is required only for the constant values $x_{2}=x_{e_{2}}$ and $x_{2}=x_{i_{2}}$. On making these substitutions and taking account of eq 1 , it is found that

$$
\begin{aligned}
M=M_{0} & +\frac{1}{4 \pi^{2} p_{1} p_{2}}\left\{W_{12}+W_{21}+\right. \\
& +\omega_{2}\left(x_{e_{2} i_{1}}, \theta_{i_{1}}, \theta_{e_{2}}\right)-\omega_{2}\left(x_{e_{2_{2}} e_{1}}, \theta_{e_{1}}, \theta_{e_{2}}\right) \\
& \left.+\omega_{2}\left(x_{i_{2} e_{1}}, \theta_{e_{1}}, \theta_{i_{2}}\right)-\omega_{2}\left(x_{i_{2} i_{1}}, \theta_{i_{1}}, \theta_{i_{2}}\right)\right\},
\end{aligned}
$$

where

$\omega_{2}\left(x_{t_{2} t_{1}}, \theta_{t_{1}}, \theta_{t_{2}}\right) \equiv$

$2 a_{1} a_{2} \int_{0}^{2 \pi} d \theta_{1} \int_{0}^{2 \pi} d \theta_{2}\left\{\left[p_{2} \theta\left(\theta_{t_{2}}-\theta_{2}\right)-p_{1} \Theta\left(\theta_{t_{1}}-\theta_{1}\right)\right]^{2}-\frac{\pi^{2}\left(p_{1}^{2}+p_{2}^{2}\right)}{12}\right\} F\left(x_{t_{2} t_{1}}, \theta_{1}, \theta_{2}\right)$

and

$x_{t_{2} t_{1}} \equiv x_{t_{2}}-x_{t_{1}}$,

where the subscript $t$ suggests "terminal," so that $x_{t 2 t 1}$ is any one of the four possible distances between terminal planes, one of which is a terminal plane for the first sheet, the other for the second sheet.

Each of the four $x$-differences represented by $x_{t_{2} t_{1}}$, namely,

$$
x_{e_{2} i_{1}}=x_{e_{2}}-x_{i_{1}}, x_{e_{2} e_{1}}=x_{e_{2}}-x_{e_{1}}, x_{i_{2} e_{1}}=x_{i_{2}}-x_{e_{1}}, x_{i_{2} i_{1}}=x_{i_{2}}-x_{i_{1}}
$$

may be positive or negative. The notation $\theta_{t_{1}}$ indicates which terminal azimuth of No. 1 , and $\theta_{t}$, which terminal azimuth of No. 2 is associated with a specified $x$-difference. 
The second-order term, $\omega_{2}$, is an even function of its $x$-argument, but the other terms are not so simple for

$$
\begin{aligned}
W_{12} \equiv \int & \int_{s_{1}} d S_{1} 2 a_{2} \int_{0}^{2 \pi} d \theta_{2} \\
& {\left[p_{2} \Theta\left(\theta_{e_{3}}-\theta_{2}\right) F\left(x_{e_{3}}-x_{1}, \theta_{1}, \theta_{2}\right)-p_{2} \Theta\left(\theta_{i_{1}}-\theta_{2}\right) F\left(x_{i_{2}}-x_{1}, \theta_{1}, \theta_{2}\right)\right] }
\end{aligned}
$$

and

$$
\begin{aligned}
W_{21} \equiv \int & \int_{s_{2}} d S_{2} 2 a_{1} \int_{0}^{2 \pi} d \theta_{1} \\
& \quad\left[p_{1} \Theta\left(\theta_{e_{1}}-\theta_{1}\right) F\left(x_{2}-x_{e_{1}}, \theta_{1}, \theta_{2}\right)-p_{1} \Theta\left(\theta_{i_{1}}-\theta_{1}\right) F\left(x_{2}-x_{i_{1}}, \theta_{1}, \theta_{2}\right)\right] .
\end{aligned}
$$

If the integrals were left in this form, it would be necessary to discriminate between the various cases arising out of the possible combinations of positive and negative signs among the four $x$-values. The formulation of these cases would be tedious and awkward because of their lack of symmetry.

This may be avoided without any loss in generality by transforming all the $x$-integrations of eq 23 into others in which the $x$-argument of $F$ has the same algebraic sign throughout the range of integration. To do this, we may write eq $23 \mathrm{a}$ in the form

$$
W_{12}=2 a_{1} a_{2} \int_{0}^{2 \pi} d \theta_{1} \int_{0}^{2 \pi} d \theta_{2}\left\{p_{2} \Theta\left(\theta_{e_{2}}-\theta_{2}\right) \int_{x_{i_{1}}}^{x_{c_{1}}} d x_{1} F\left(x_{e_{2}}-x_{1}, \theta_{1}, \theta_{2}\right)\right.
$$

The first $x_{1}$ integral is $\left.\quad-p_{2} \Theta\left(\theta_{i_{2}}-\theta_{2}\right) \int_{x_{i_{1}}}^{x_{\epsilon_{1}}} d x_{1} F\left(x_{i_{8}}-x_{1}, \theta_{1}, \theta_{2}\right)\right\}$

$$
\int_{x_{i_{1}}}^{x_{e_{1}}} F\left(x_{e_{1}}-x_{1}\right) d x_{1}=\int_{0}^{x_{e_{g} i_{1}}} F\left(x^{\prime}\right) d x^{\prime}-\int_{0}^{x_{e_{g_{1} \varepsilon_{1}}}} F\left(x^{\prime}\right) d x^{\prime} .
$$

The second is

$$
\int_{x_{i_{1}}}^{x_{\varepsilon_{1}}} F\left(x_{i_{2}}-x_{1}\right) d x_{1}=\int_{0}^{x_{i_{2} i_{1}}} F\left(x^{\prime}\right) d x^{\prime}-\int_{0}^{x_{i_{2} e_{1}}} F\left(x^{\prime}\right) d x^{\prime},
$$

so that

$$
\begin{array}{r}
W_{12}=2 a_{1} a_{2} \int_{0}^{2 \pi} d \theta_{1} \int_{0}^{2 \pi} d \theta_{2}\left\{p_{2} \Theta\left(\theta_{e_{2}}-\theta_{2}\right)\left[\int_{0}^{x_{e_{2} i_{1}}} F\left(x^{\prime}, \theta_{1}, \theta_{2}\right) d x^{\prime}-\int_{0}^{x_{c_{2} \varepsilon_{1}}} F\left(x^{\prime}, \theta_{1}, \theta_{2}\right) d x^{\prime}\right]\right. \\
\left.+p_{2} \Theta\left(\theta_{i_{1}}-\theta_{2}\right)\left[\int_{0}^{x_{i_{g_{1}}}} F\left(x^{\prime}, \theta_{1}, \theta_{2}\right) d x^{\prime}-\int_{0}^{x_{i_{2} i_{1}}} F\left(x^{\prime}, \theta_{1}, \theta_{2}\right) d x^{\prime}\right]\right\} .
\end{array}
$$


Similarly,

$$
\begin{gathered}
W_{21}= \\
2 a_{1} a_{2} \int_{0}^{2 \pi} d \theta_{1} \int_{0}^{2 \pi} d \theta_{2}\left\{p_{1} \theta\left(\theta_{c_{1}}-\theta_{1}\right)\left[\int_{0}^{x_{22_{1} p_{1}}} F\left(x^{\prime}, \theta_{1}, \theta_{2}\right) d x^{\prime}-\int_{0}^{x_{i_{2} \theta_{1}}} F\left(x^{\prime}, \theta_{1}, \theta_{2}\right) d x^{\prime}\right]\right. \\
+p_{1} \theta\left(\theta_{i_{1}}-\theta_{1}\right)\left[\int_{0}^{x_{i_{2} i_{1}}} F\left(x^{\prime}, \theta_{1}, \theta_{2}\right) d x^{\prime}-\int_{0}^{x_{a_{2} i_{1}}} F\left(x^{\prime}, \theta_{1}, \theta_{2}\right) d x^{\prime}\right] .
\end{gathered}
$$

Adding these gives

$$
\begin{gathered}
W_{12}+W_{21}= \\
=\omega_{1}\left(x_{e_{2} i_{1}}, \theta_{i_{1}}, \theta_{e_{2}}\right)-\omega_{1}\left(x_{e_{2} e_{1}}, \theta_{e_{1}}, \theta_{e_{2}}\right)+\omega_{1}\left(x_{i_{2} e_{1}}, \theta_{e_{1}}, \theta_{i_{2}}\right)-\omega_{1}\left(x_{i_{2} i_{1}}, \theta_{i_{1}}, \theta_{i_{2}}\right),
\end{gathered}
$$

where

$$
\begin{gathered}
\omega_{1}\left(x_{t_{2} t_{1}}, \theta_{t_{1}}, \theta_{t_{2}}\right) \equiv \\
2 a_{1} a_{2} \int_{0}^{2 \pi} d \theta_{1} \int_{0}^{2 \pi} d \theta_{2}\left[p_{2} \Theta\left(\theta_{t_{2}}-\theta_{2}\right)-p_{1} \Theta\left(\theta_{t_{1}}-\theta_{1}\right)\right] \int_{0}^{x_{t_{n_{1}}}} F\left(x^{\prime}, \theta_{1}, \theta_{2}\right) d x^{\prime} .
\end{gathered}
$$

This definition makes $\omega_{1}$ an odd function of its $x$ argument.

The mutual inductance of the current sheets may be put in a similar form by writing the $x_{1} x_{2}$ integrals of eq 1

$$
\int_{x_{i_{1}}}^{x_{x_{1}}} d x_{1} \int_{x_{i_{2}}}^{x_{c_{2}}} d x_{2} F\left(x_{2}-x_{1}\right)=\int_{x_{i_{1}}}^{x_{c_{1}}} d x_{1} \int_{0}^{x_{c_{2}}-x_{1}} F\left(x^{\prime}\right) d x^{\prime}-\int_{x_{i_{1}}}^{x_{x_{1}}} d x_{1} \int_{0}^{x_{i_{2}}-x_{1}} F\left(x^{\prime}\right) d\left(x^{\prime}\right) .
$$

In the first of these double integrals let $x^{\prime \prime}=x_{e_{3}}-x_{1}$, and in the second let $x^{\prime \prime}=x_{i_{2}}-x_{1}$. The first becomes

$$
\begin{aligned}
& \int_{x_{i_{1}}}^{x_{\theta_{1}}} d x_{1} \int_{0}^{x_{c_{2}}-x_{1}} F\left(x^{\prime}\right) d x^{\prime}=\int_{x_{c_{2}} x_{c_{1}}}^{x_{x_{2}}-x_{i_{1}}} d \int_{0}^{x^{\prime \prime}} F\left(x^{\prime}\right) d x^{\prime} \\
& =\int_{0}^{x_{02 i_{1}}} d x^{\prime \prime} \int_{0}^{x^{\prime \prime}} F\left(x^{\prime}\right) d x^{\prime}-\int_{0}^{x_{02 x_{1}}} d x^{\prime \prime} \int_{0}^{x^{\prime \prime}} F\left(x^{\prime}\right) d x^{\prime} .
\end{aligned}
$$

The second becomes

$$
-\int_{x_{i_{1}}}^{x_{e_{1}}} d x_{1} \int_{0}^{x_{i_{2}}-x_{1}} F\left(x^{\prime}\right) d x^{\prime}=\int_{0}^{x_{i_{2} \vartheta_{1}}} d x^{\prime \prime} \int_{0}^{x^{*}} F\left(x^{\prime}\right) d x^{\prime}-\int_{0}^{x_{i_{2} i_{1}}} d x^{\prime \prime} \int_{0}^{x^{*}} F\left(x^{\prime}\right) d x^{\prime} .
$$

Hence eq 1 gives as the mutual inductance, $M_{0}$, of the two current sheets

where

$$
M_{0}=\frac{1}{4 \pi^{2} p_{1} p_{2}}\left\{\omega_{0}\left(x_{e_{2} i_{1}}\right)-\omega_{0}\left(x_{e_{2} e_{1}}\right)+\omega_{0}\left(x_{i_{2} e_{1}}\right)-\omega_{0}\left(x_{i_{2} i_{1}}\right)\right\},
$$

$$
\omega_{0}(x) \equiv a_{1} a_{2} \int_{0}^{2 \pi} d \theta_{1} \int_{0}^{2 \pi} d \theta_{2} \int_{0}^{x} d x^{\prime \prime} \int_{0}^{x^{\prime \prime}} d x^{\prime} F\left(x^{\prime}, \theta_{1}, \theta_{2}\right),
$$

which is an even function of $x$ vanishing with $x$. Its $x$ derivative, $\omega_{0}^{\prime}(x)$, is an odd function of $x$ vanishing with it. 
An interpretation of the function, $\omega_{0}(x)$, may be obtained by considering the special case where $x_{i_{1}}=x_{i_{2}}$ and $x_{e_{1}}=x_{e_{2}}$, which is

where

$$
M=\frac{2 \omega_{0}(x)}{4 \pi^{2} p_{1} p_{2}}=2 n_{1} n_{2} \omega_{0}(x),
$$

$$
x=x_{e_{2} i_{1}}=x_{i_{2} e_{1}}=l_{1}=l_{2}
$$

and $n_{1}=1 / 2 \pi p_{1}, n_{2}=1 / 2 \pi p_{2}=$ number of turns per unit axial length. This shows that $\omega_{0}(x)$ is one-half the mutual inductance of the two current sheets, when they have the same end planes, their common length being $|x|$, and when each sheet has one turn per unit axial length (both axial and angular current in the sheets).

Collecting these results, the mutual inductance, $M$, between the two helices (their Neumann's integral) is

$$
\begin{array}{r}
M=n_{1} n_{2}\left\{\omega\left(x_{e_{2} i_{1}}, \theta_{i_{1}}, \theta_{e_{2}}\right)-\omega\left(x_{e_{2} e_{1}}, \theta_{e_{1}}, \theta_{e_{2}}\right)+\omega\left(x_{i_{2} e_{1}}, \theta_{e_{1}}, \theta_{i_{2}}\right)\right. \\
\left.-\omega\left(x_{i_{2} i_{1}}, \theta_{i_{1}}, \theta_{i_{2}}\right)\right\},
\end{array}
$$

where

$$
\omega\left(x_{t_{2} t_{1}}, \theta_{t_{1}}, \theta_{t_{2}}\right)=\omega_{0}\left(x_{t_{2} t_{1}}\right)+\omega_{1}\left(x_{t_{2} t_{1}}, \theta_{t_{1}}, \theta_{t_{2}}\right)+\omega_{2}\left(x_{t_{2} t_{1}}, \theta_{t_{1}}, \theta_{t_{2}}\right),
$$

where $\omega_{0}$ is given by eq $26^{1}, \omega_{1}$ by eq 25 , and $\omega_{2}$ by eq 21 . The $x$-differences, $x_{t_{2} t_{1}}$, between terminal planes of the current sheets and the terminal azimuths, $\theta_{t_{1}}$ and $\theta_{t_{2}}$, associated with each of these are given in eq 22 and $22^{1}$. It is now apparent that eq 28 is general, whatever combination of positive and negative signs the four $x$-differences may have. These signs are automatically taken care of in the definitions, eq $26^{1}, 25$, and 21 , of $\omega_{0}, \omega_{1}$, and $\omega_{2}$, respectively, which shows that and $\omega_{0}$ and $\omega_{2}$ are continuous even functions of $x, \omega_{1}$ being an odd function of $x$.

It is evident also from these definitions, that $\omega_{1}$ and $\omega_{2}$ (and hence $\omega$ ) are periodic functions of the terminal azimuths $\theta_{t_{1}}$ and $\theta_{t_{2}}$, since $\theta$ is periodic with period $2 \pi$. The period of $\omega$ will be seen to be $2 \pi$ for each angle. Consequently, $\omega\left(x_{t_{1} t_{2}}, \theta_{t_{1}}, \theta_{t_{2}}\right)$ is developable in a double Fourier's series of sines and cosines of multiples of these two angles, the development holding for all values of the angles and the coefficients being functions of $x_{t_{2} t_{1}}$ (and of $r, a_{1}, a_{2}$ )

Since $\omega_{0}$ is the finite term, both the terms $\left(\cos \left(\theta_{2}-\theta_{1}\right)\right.$ and $p_{1} p_{2} / a_{1} a_{2}$ (constituting the numerator of the expression (eq 2) defining $F^{\prime}$ ) must be retained in evaluating $\omega_{0}$. But since $\omega_{1}$ is in general an infinitesimal of first order and $\omega_{0}$ of second order, the term $p_{1} p_{2} / a_{1} a_{2}$ is negligible in the definition of $F$, which is used for evaluating $\omega_{1}$ and $\omega_{2}$ (since terms of third order have been neglected).

The system of subscripts in the designation of the three associated variables $\left(x_{t_{2} t_{1}}, \theta_{t_{1}}, \theta_{t_{2}}\right)$ has now served its purpose of indicating which pair of terminal azimuths is associated with a given $x$-difference. From here on in the study of the $\omega$ functions we adopt the simpler notation $\left(x, \theta_{1}, \theta_{2}\right)$ for these three associated variables and place here for reference, see eq 22 and $22^{\prime}$,

$$
\left\{\begin{array}{l}
x=x_{t_{1} t_{2}}, \theta_{1}=\theta_{t_{1}}, \theta_{2}=\theta_{t_{2}} \\
\omega\left(x_{1} \theta_{1}, \theta_{2}\right)=\omega\left(x_{t_{1} t_{2}}, \theta_{t_{1}}, \theta_{t_{2}}\right)
\end{array}\right\} .
$$


This change necessitates a change in the notation of the variables of integration so that eq $26^{\prime}, 25$, and 21 may now be written

$$
\begin{gathered}
\omega_{0}(x)=a_{1} a_{2} \int_{0}^{x} d x^{\prime \prime} \int_{0}^{x^{\prime \prime}} d x^{\prime} \int_{0}^{2 \pi} d \theta_{1}^{\prime} \int_{0}^{2 \pi} d \theta_{2} \frac{\left[\cos \left(\theta_{1}{ }^{\prime}-\theta_{2}{ }^{\prime}\right)+p_{1} p_{2} / a_{1} a_{2}\right]}{R_{12}\left(x^{\prime}, \theta_{1}{ }^{\prime}, \theta_{2}{ }^{\prime}\right)} \\
\omega_{1}\left(x, \theta_{1}, \theta_{2}\right)=
\end{gathered}
$$

$2 a_{1} a_{2} \int_{0}^{x} d x^{\prime} \int_{0}^{2 \pi} d \theta_{1} \int_{0}^{2 \pi} d \theta_{2}{ }^{\prime}\left[p_{2} \Theta\left(\theta_{2}-\theta_{2}{ }^{\prime}\right)-p_{1} \Theta\left(\theta_{1}-\theta_{1}{ }^{\prime}\right)\right] \frac{\cos \left(\theta_{1}{ }^{\prime}-\theta_{2}{ }^{\prime}\right)}{R_{12}\left(x^{\prime}, \theta_{1}{ }^{\prime}, \theta_{2}{ }^{\prime}\right)}$

$\omega_{2}\left(x, \theta_{1}, \theta_{2}\right)=2 a_{1} a_{2} \int_{0}^{2 \pi} d \theta_{1}^{\prime} \int_{0}^{2 \pi} d \theta_{2}^{\prime}\left\{p_{1}^{2}\left[\theta^{2}\left(\theta_{1}-\theta_{1}{ }^{\prime}\right)-\frac{\pi^{2}}{12}\right]\right.$

$$
\left.+p_{2}^{2}\left[\theta^{2}\left(\theta_{2}-\theta_{2}^{\prime}\right)-\frac{\pi^{2}}{12}\right]-2 p_{1} p_{2} \theta\left(\theta_{1}-\theta_{1}^{\prime}\right) \theta\left(\theta-\theta_{2}^{\prime}\right)\right\} \frac{\cos \left(\theta_{1}^{\prime}-\theta_{2}^{\prime}\right)}{R_{12}\left(x, \theta_{1}^{\prime}, \theta_{2}^{\prime}\right)} .
$$

Equation 28 becomes

$$
\omega\left(x, \theta_{1}, \theta_{2}\right)=\omega_{0}(x)+\omega_{1}\left(x, \theta_{1}, \theta_{2}\right)+\omega_{2}\left(x, \theta_{1}, \theta_{2}\right) .
$$

In the relatively small terms $\omega_{1}$ and $\omega_{2}$, a sufficient approximation to the exact expressions

is

$$
\theta(\theta)=\sum_{n=1}^{\infty} \frac{\sin n \theta}{n} \text { and } \theta^{2}(\theta)-\frac{\pi^{2}}{12}=\sum_{n=1}^{\infty} \frac{\cos n \theta}{n^{2}}
$$

$$
\theta(\theta)=\frac{\pi^{2}}{9}\left(\sin \theta+\frac{1}{2} \sin 2 \theta\right) \text { and } \theta^{2}(\theta)-\frac{\pi^{2}}{12}=\frac{\pi^{2}}{9}\left(\cos \theta+\frac{\cos 2 \theta}{4}\right) .
$$

Using this approximation, eq 31 becomes

$$
\begin{array}{r}
\omega_{1}\left(x, \theta_{1}, \theta_{2}\right)=\frac{2 \pi^{2}}{9} a_{1} a_{2} \int_{0}^{x} d x^{\prime} \int_{0}^{2 \pi} d \theta_{1}^{\prime} \int_{0}^{2 \pi} d \theta_{2}^{\prime}\left\{p_{1}\left[\sin \left(\theta_{1}^{\prime}-\theta_{1}\right)+\frac{1}{2} \sin 2\left(\theta_{1}^{\prime}-\theta_{1}\right)\right]\right. \\
-p_{2}\left[\sin \left(\theta_{2}^{\prime}-\theta_{2}\right)+\frac{1}{2} \sin 2\left(\theta_{2}^{\prime}-\theta_{2}\right)\right] \frac{\cos \left(\theta_{1}^{\prime}-\theta_{2}^{\prime}\right)}{R_{12}\left(x^{1}, \theta_{1}^{\prime}, \theta_{2}^{\prime}\right.}
\end{array}
$$

and eq 32 becomes

$$
\begin{array}{r}
\omega_{2}\left(x, \theta_{1}, \theta_{2}\right)=\frac{2 \pi^{2} a_{1} a_{2}}{9} \int_{0}^{2 \pi} d \theta_{1}^{\prime} \int_{0}^{2 \pi} d \theta_{2}^{\prime}\left\{p_{1}^{2}\left[\cos \left(\theta_{1}^{\prime}-\theta_{1}\right)+\frac{1}{4} \cos 2\left(\theta_{1}^{\prime}-\theta_{1}\right)\right]\right. \\
+p_{2}^{2}\left[\cos \left(\theta_{2}^{\prime}-\theta_{2}\right)+\frac{1}{4} \cos 2\left(\theta_{2}^{\prime}-\theta_{2}\right)\right]
\end{array}
$$

$-\frac{2 \pi^{2} p_{1} p_{2}}{9}\left[\sin \left(\theta_{1}^{\prime}-\theta_{1}\right)+\frac{1}{2} \sin 2\left(\theta_{1}^{\prime}-\theta_{1}\right)\right]$

$$
\left[\sin \left(\theta_{2}^{\prime}-\theta_{2}\right)+\frac{1}{2} \sin 2\left(\theta_{2}^{\prime}-\theta_{2}\right] \frac{\cos \left(\theta_{1}^{\prime}-\theta_{2}^{\prime}\right)}{R_{12}\left(x, \theta_{1}^{\prime}, \theta_{2}^{\prime}\right)} .\right.
$$

For reference is placed here an alternative approximation

$$
\left.\begin{array}{rl}
\theta^{2}(\theta) & =\frac{\pi^{2}}{4}\left(1-\sin \frac{\theta}{2}\right), \text { when } 0<\theta<2 \pi \\
& =\frac{\pi^{2}}{4}\left(1+\sin \frac{\theta}{2}\right), \text { when }-2 \pi<\theta<0
\end{array}\right\},
$$


which is seen to be a good approximation, since

and

$$
32 / \pi^{3}=1.032
$$

$$
\theta^{2}(\theta)=\frac{\pi^{2}}{4}\left[1-\frac{32}{\pi^{3}} \sum_{n=1}^{\infty} \frac{\sin (2 n-1) \frac{\theta}{2}}{(2 n-1)^{3}}\right], \text { when } 0<\theta<\pi / 2 .
$$

For the type of expansions required here the three-term approximation for $\theta^{2}$ given in eq 35 will be used. It has about the same precision as the two-term approximation, eq 38 . The latter is equivalent to one used ${ }^{3}$ in considering coaxial helices, as it led to a simple expression for the term corresponding in that case to $\omega_{2}$, which was in finite terms.

A comparison of the approximate expression adopted for $\theta(\theta)$ and for $\theta^{2}(\theta)-\mathrm{II}^{2} / 12$ in (eq 35) with the exact series (eq 34), for these functions shows that we have retained only the first two terms of the series, and have arbitrarily inserted the factor $\pi^{2} / 9$, which is slightly greater than 1 , and in fact it is sufficient to replace this by 1 in the secondorder terms.

In the following section the $\omega$-functions are expanded in a double Fourier's series in the terminal azimuth angles $\theta_{1}$ and $\theta_{2}$.

\section{EXPANSION OF $\omega$ IN A DOUBLE FOURIER'S SERIES OF THE AZIMUTHAL ANGLES}

In figure 3 is shown the projection of the two parallel cylinders upon the $y z$ plane, and $r_{12}$ is the projection of the distance $R_{12}$ from $P_{1}$ to $P_{2}$, so that

$$
R_{12}^{2}=x^{2}+r_{12}^{2} \text {, where } x=x_{2}-x_{1} .
$$

The $x$ axis being taken as the axis of cylindrical coordinates, $a_{1}$ and $\theta_{1}$ are cylindrical coordinates of $P_{1}$, but the cylindrical coordinates of $P_{2}$ may be taken as $r_{2}$ and $\theta_{2}^{\prime}$, as shown in figure 3 . Since $r_{12}$ is one side of the triangle $P_{1} O_{1} P_{2}$, the other two sides, $a_{1}$, and $r_{2}$, including the angle $\theta_{1}-\theta_{2}^{\prime}$,

$$
r_{12}^{2}=a_{1}^{2}-2 a_{1} r_{2} \cos \left(\theta_{1}-\theta_{2}^{\prime}\right)+r_{2}^{2} .
$$

Since $1 / R_{12}$ is an even periodic function of the angle $\left(\theta_{1}-\theta_{2}^{\prime}\right)$, its Fourier series may be written

$$
\frac{1}{R_{12}}=\frac{2}{\sqrt{a_{1} r_{2}}} \sum_{n=0}^{\infty} \epsilon_{n} \phi_{n}\left(x, a_{1}, r_{2}\right) \cos n\left(\theta_{1}-\theta_{2}^{\prime}\right),
$$

where $\epsilon_{0}=\frac{1}{2}$ and $\epsilon_{n}=1$, if $n \neq 0$ ), and where

$$
\frac{\phi_{n}\left(x, a_{1}, r_{2}\right)}{\sqrt{a_{1} r_{2}}}=\frac{1}{2 \pi} \int_{0}^{2 \pi} \frac{\cos n\left(\theta_{1}-\theta_{2}^{\prime}\right) d \theta_{1}}{R_{12}}
$$

and $x, a_{1}, r_{2}, \theta_{2}^{\prime}$ are held constant in this integration.

\footnotetext{
3 Reference 2, p. 259-261.
} 
Another integral representation of this function, which will be derived later and which involves Bessel's function, $J_{n}$, is

$$
\frac{\phi_{n}\left(x, a_{1}, r_{2}\right)}{\sqrt{a_{1} r_{2}}}=\int_{0}^{\infty} e^{\infty}|x| t J_{n}\left(a_{1} t\right) J_{n}\left(r_{2} t\right) d t .
$$

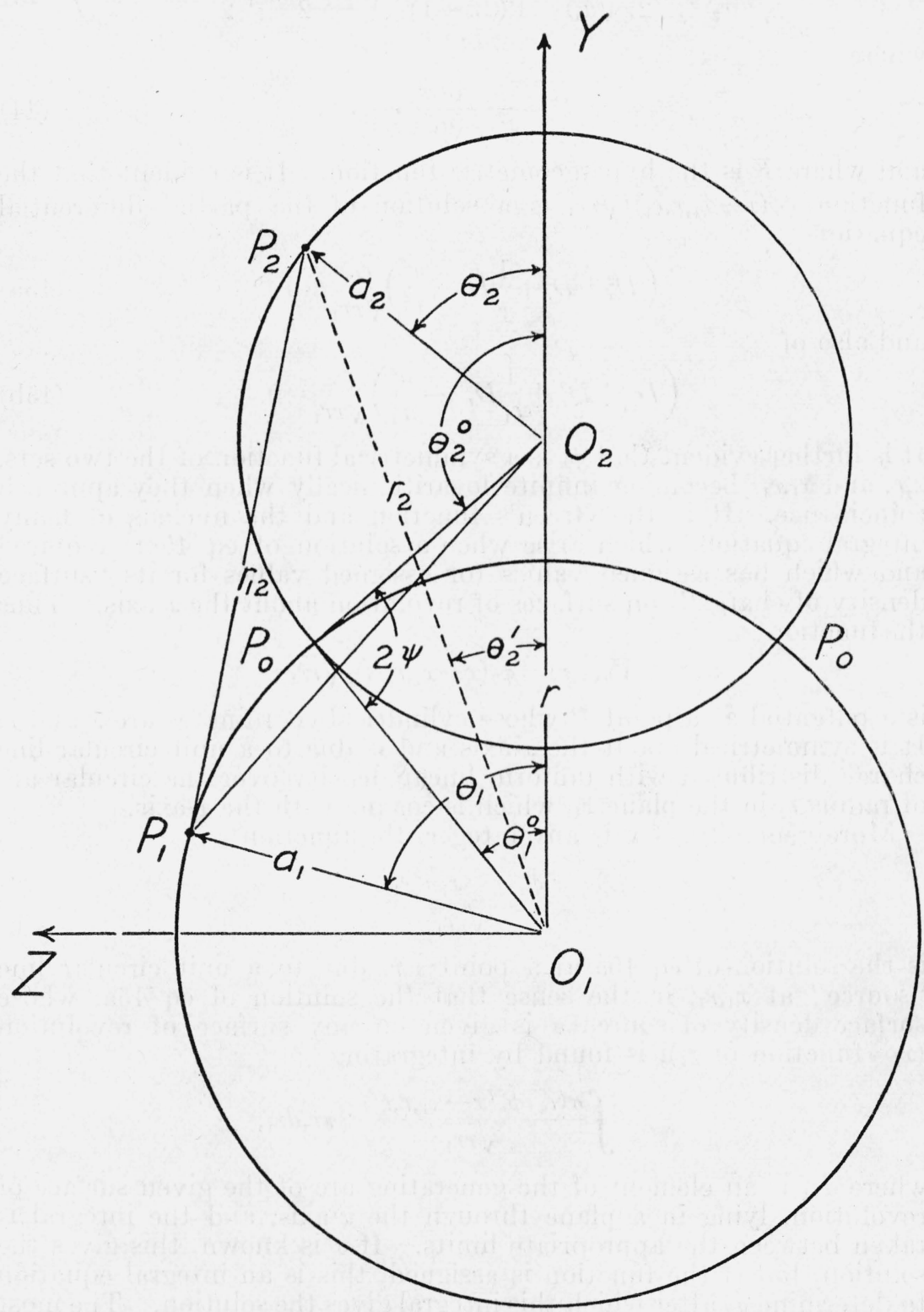

Figdre 3.-Projection of the two helices upon a plane perpendicular to their axes. 
This integral may be evaluated, the result being a function of the single dimensionless variable $k$, so that

$$
\frac{\phi_{n}\left(x, a_{1}, r_{2}\right)}{\sqrt{a_{1} r_{2}}}=\frac{k^{2 n+1}}{2 \pi \sqrt{a_{1} r_{2}}} \cdot \frac{\Gamma^{2}\left(n+\frac{1}{2}\right)}{\Gamma(2 n+1)} F\left(n+\frac{1}{2}, n+\frac{1}{2}, 2 n+1 ; k^{2}\right),
$$

where

$$
k^{2}=\frac{4 a_{1} r_{2}}{x^{2}+\left(a_{1}+r_{2}\right)^{2}},
$$

and where $F$ is the hypergeometric function. It is evident that the function $\phi_{n}\left(x-x_{1}, r, r_{1}\right) / \sqrt{r r_{1}}$ is a solution of the partial differential equation

and also of

$$
\left(D_{x}^{2}+D_{r}^{2}+\frac{1}{r} D_{r}-\frac{n^{2}}{r^{2}}\right) \frac{\phi_{n}}{\sqrt{r r_{1}}}=0
$$

$$
\left(D_{x_{1}}^{2}+D_{r_{1}}^{2}+\frac{1}{r_{1}} D_{r_{-}}-\frac{n^{2}}{r_{1}^{2}}\right) \frac{\phi_{n}}{\sqrt{r r_{1}}}=0 .
$$

It is further evident that $\phi_{n}$ is a symmetrical function of the two sets, $x, r$, and $x_{1}, r_{1}$, becoming infinite logarithmically when they approach coincidence. It is the Green's function and the nucleus of many integral equations which arise when a solution of eq 45 is required and which has assigned values (or assigned values for its "surface density of charge") on surfaces of revolution about the $x$-axis. Thus the function

$$
V_{0}(x, r)=\phi_{0}\left(x-x_{1}, r, r_{1}\right) / \sqrt{r r_{1}}
$$

is a potential at a point $P$ whose cylindrical coordinates are $x$ and $r$. It is symmetrical about the $x$-axis and is due to a unit circular line charge distributed with uniform linear density over the circular arc of radius $r_{1}$ in the plane $x_{1}$, which is coaxial with the $x$-axis.

More generally, if $n$ is any integer, the function

$$
\frac{\phi_{n}\left(x-x_{1}, r, r_{1}\right)}{\sqrt{r r_{1}}}
$$

is the solution of eq $45 \mathrm{a}$ at a point $x, r$, due to a unit circular line "source" at $x_{1}, r_{1}$, in the sense that the solution of eq $45 \mathrm{a}$, whose surface density of source $\sigma$ is given on any surface of revolution $\left(x_{1}=\right.$ function of $\left.r_{1}\right)$, is found by integrating

$$
\int \frac{\sigma\left(r_{1}\right) \phi_{n}\left(x-x_{1}, r, r_{1}\right)}{\sqrt{r r_{1}}} 2 \pi r_{1} d s_{1},
$$

where $d s_{1}$ is an element of the generating arc of the given surface of revolution, lying in a plane through the $x$-axis, and the integral is taken between the appropriate limits. If $\sigma$ is known, this gives the solution, but if the function is assigned, this is an integral equation to determine $\sigma$, after which this integral gives the solution. The most appropriate expansions of this nucleus of the integral equations in a given coordinate system are those which are the canonical bilinear expansion of the nucleus in terms of its normal functions for that system. 
The role of this function $\phi_{n}$ for solutions of eq $45 \mathrm{a}$, having assigned values on surfaces of revolution, is as fundamental as that of $1 / R_{p_{1} p}$ in the general theory of electrostatics, and in fact its expansion in normal functions follows from that of $1 / R_{p_{1} p}$ by eq $42 \mathrm{a}$.

Some coordinates pertinent to the problem of this paper may here be mentioned.

The first is the spherical coordinate system. If the values of a solution $U$ of eq $45 \mathrm{a}$ are assigned on the sphere made by revolving the circle $x_{1}^{2}+r_{1}^{2}=R_{1}^{2}$ about the $x$-axis, and $R, \zeta, \theta$ are the spherical coordinates of $P$, while $\left(R_{1}, \zeta_{1}, \theta_{1}\right)$ are those of $P_{1}$, where $R=x^{2}+r^{2}$, $\zeta=x / R$ and $\tan \theta=y / z$ (where $\theta$ is the same as in cylindrical coordinates), then it is known that

$\frac{1}{R_{P_{1} P}}=2 \sum_{n=0}^{\infty} \epsilon_{n} \cos n\left(\theta-\theta_{1}\right) \sum_{m=n}^{\infty} \frac{\Gamma(m-n+1)}{\Gamma(m+n+1)} P_{m}^{n}(\zeta) R^{-m-1} P_{m}^{n}\left(\zeta_{1}\right) R_{1}{ }^{m}$

when $R>R_{1}$. (with interchange of $R$ and $R_{1}$ for the case $R<R_{1}$ ).

The expansion corresponding to eq 41 is

$$
\frac{1}{R_{P_{1} P}}=\frac{2}{\sqrt{r_{1} r}} \sum_{n=0}^{\infty} \epsilon_{n} \phi_{n}\left(x-x_{1}, r, r_{1}\right) \cos n\left(\theta-\theta_{1}\right),
$$

so that

$$
\frac{\phi_{n}\left(x-x_{1}, r, r_{1}\right)}{\sqrt{r r_{1}}}=\sum_{m=n}^{\infty} \frac{\Gamma(m-n+1)}{\Gamma(m+n+1)} P_{m}^{n}(\xi) R^{-m-1} P_{m}^{n}\left(\xi_{1}\right) R_{1}^{m}
$$

when $R>R_{1}$ (with interchange of $R$ and $R_{1}$ when $R<R_{1}$ ), where $P_{m}^{n}(\xi)$ is the associated Legendre function (Ferrer's). This is the canonical bilinear expansion of the nucleus in terms of normal functions for the spherical surface $R_{1}$ by means of which solutions of eq 45a may be found when their values or the values of their source density on the sphere are assigned.

When the solution of eq $45 \mathrm{a}$ has assigned values on an oblate spheroid, oblate spheroidal coordinates are used. The bilinear expantion for this nucleus in such coordinates is used in a later section and applied to the limiting case where the spheroid becomes a circular disk.

The expansion in toroidal coordinates is also intimately related to the present problem, the function $\phi_{n}(k)$ being a toroidal function $\phi_{n}(k)=\frac{1}{\pi} Q_{n-\frac{1}{2}}\left(\frac{2}{k^{2}}-1\right)$, where $Q_{n}$ is Legendre's function of the second kind.

The derivation of eq $42 \mathrm{~b}$ may be made by use of Heine's integral formula,

$$
\frac{1}{R_{12}}=\frac{1}{\sqrt{x^{2}+r_{12}^{2}}}=\int^{\infty} e^{-|x| t} J_{0}\left(r_{12} t\right) d t .
$$

Using Neumann's addition formula,

$$
\begin{aligned}
& J_{0}\left(r_{12} t\right)=\sum_{n_{1}=-\infty}^{\infty} J_{n_{1}}\left(a_{1} t\right) J_{n_{1}}\left(r_{2} t\right) e^{i n_{1}\left(\theta_{1}-\theta_{2}^{\prime}\right)} \\
& =2 \sum_{n=0}^{\infty} \epsilon_{n} J_{n}\left(a_{1} t\right) J_{n}\left(r_{2} t\right) \cos n\left(\theta_{1}-\theta_{2}^{\prime}\right)
\end{aligned}
$$


gives

$$
\frac{1}{R_{12}}=2 \sum_{n=0}^{\infty} \epsilon_{n} \cos n\left(\theta_{1}-\theta_{2}^{\prime}\right) \int_{0}^{\infty} e^{-|x| t} . J_{n}\left(a_{1} t\right) J_{n}\left(r_{2} t\right) d t,
$$

which, by comparison with eq 41 , gives eq $42 \mathrm{~b}$. In the expansion (eq 41) or its equivalent (eq 51), both $r_{2}$ and $\theta_{2}^{\prime}$ are functions of $\theta_{2}$. Since $r_{2}$ is one side of the triangle, $P_{2} O_{1} O_{2}$ (fig. 3), the other sides, $r$ and $a_{2}$, including the angle $\left(\pi-\theta_{2}\right)$,

$$
r_{2}^{2}=r^{2}-2 r a_{2} \cos \left(\pi-\theta_{2}\right)+a_{2}^{2} \text {. }
$$

To obtain the Fourier's cosine series in $\theta_{1}$ and $\theta_{2}$ for $1 / R_{12}$, the term $J_{0}\left(r_{12} t\right)$ in the integrand of eq 49 must be expressed explicitly in terms of these angles. Hence in the expansion (eq 50), the term $\mathrm{J}_{n_{1}}\left(r_{2} t\right) e^{-i n_{2} \theta_{2}^{\prime}}$ must be expanded as a Fourier series in $\theta_{2}$, and for this a more general formula than Neumann's addition formula (eq 50) is required.

Neumann's addition formula is a special case of a more general addition formula derivable from that of Sonin, ${ }^{4}$ which, applied to the triangle $P_{2} O_{1} O_{2}$, may be put in the form

$$
J_{n_{1}}\left(r_{2} t\right) e^{-i n_{1} \theta_{3}^{\prime}}=\sum_{n_{2}=-\infty}^{\infty} J_{n_{1}-n_{2}}(r t) J_{n_{2}}\left(a_{2} t\right) e^{-i n_{2} \theta_{2}}
$$

Using this in eq 50 gives

$$
J_{0}\left(r_{12} t\right)=\sum_{n_{1}=-\infty}^{\infty} \sum_{n_{2}=-\infty}^{\infty} J_{n_{1}-n_{2}}(r t) J_{n_{1}}\left(a_{1} t\right) J_{n_{2}}\left(a_{2} t\right) \cdot e^{i\left(n_{1} \theta_{1}-n_{2} \theta_{2}\right)} .
$$

By use of this expansion in eq 49, the double Fourier's series expansion of the function $1 / R_{12}$ defined in eq 3 is found to be

$$
\begin{aligned}
\frac{1}{R_{12}\left(x, \theta_{1}, \theta_{2}\right)} & =\frac{1}{\sqrt{a_{1} a_{2}}} \sum_{n_{1}=-\infty}^{\infty} \sum_{n_{3}=-\infty}^{\infty} \phi_{n_{1}, n_{2}}\left(x, r, a_{1}, a_{2}\right) e^{i\left(n_{1} \theta_{1}-n_{2} \theta_{2}\right)} \\
& =\frac{1}{\sqrt{a_{1} a_{2}}} \sum_{n_{1}=-\infty}^{\infty} \sum_{n_{3}=-\infty}^{\infty} \phi_{n_{1}, n_{2}}\left(x, r, a_{1}, a_{2}\right) \cos \left(n_{1} \theta_{1}-n_{2} \theta_{2}\right),
\end{aligned}
$$

which may be written

$$
\begin{aligned}
\frac{1}{R_{12}\left(x, \theta_{1}, \theta_{2}\right)}=\frac{2}{\sqrt{a_{1} a_{2}}}\left\{\frac{\phi_{00}}{2}+\right. & \sum_{n_{1}=1}^{\infty} \phi_{n_{1}, n_{1}} \cos n_{1}\left(\theta_{2}-\theta_{1}\right) \\
& \left.+\sum_{n=1}^{\infty} \sum_{n_{3}=-\infty}^{\infty} \phi_{n+n_{2}, n_{2}} \cos \left[\left(n+n_{2}\right) \theta_{1}-n_{2} \theta_{2}\right]\right\}
\end{aligned}
$$

or

$$
\begin{aligned}
& \frac{1}{R_{12}\left(x, \theta_{1}, \theta_{2}\right)}= \\
& \begin{aligned}
\frac{2}{\sqrt{a_{1} a_{2}} \sum_{1}=0} \sum_{n_{3}=0}^{\infty} \epsilon_{n_{1}} \epsilon_{n_{2}}\left\{\left(\phi_{n_{1}, n_{2}}+\phi_{n_{1},-n_{2}}\right) \cos n_{1} \theta_{1} \cos n_{2} \theta_{2}\right. \\
\\
\left.\quad+\left(\phi_{n_{1}, n_{2}}-\phi_{n_{1},-n_{2}}\right) \sin n_{1} \theta_{1} \sin n_{2} \theta_{2}\right\}
\end{aligned}
\end{aligned}
$$

' N. Nielsen, Handbuch der Theorie der Cylinder Funktionen, p. 287, Teubner (1904). 

by

The coefficients $\phi n_{1}, n_{2}$ are even functions of $x$, which are represented

$$
\phi_{n_{1}, n_{2}}\left(x, r, a_{1}, a_{2}\right)=\sqrt{a_{1} a_{2}} \int_{0}^{\infty} e^{-|x| t} J_{n_{1}-n_{2}}(r t) J_{n_{1}}\left(a_{1} t\right) J_{n_{2}}\left(a_{2} t\right) d t \text {, }
$$

or by Fourier's rule,

$$
\phi_{n_{1}, n_{2}}\left(x, r, a_{1}, a_{2}\right)=\frac{\sqrt{a_{1} a_{2}}}{4 \pi^{2}} \int_{0}^{2 \pi} d \theta_{1} \int_{0}^{2 \pi} d \theta_{2} \frac{\cos \left(n_{1} \theta_{1}-n_{2} \theta_{2}\right)}{R_{12}\left(x, \theta_{1}, \theta_{2}\right)},
$$

which shows that $\phi_{n_{1},-n_{2}}=\phi_{-n_{1}, n_{2}}$. On inserting the expansion (eq 56) in the three equations, $30,31,32$, noting that

$$
\begin{aligned}
& \int_{0}^{2 \pi} \theta_{1}\left(\theta-\theta_{1}^{\prime}\right) \cos n \theta_{1}^{\prime} d \theta_{1}^{\prime}=\frac{\pi}{n} \sin n \theta_{1}, \text { if } n \neq 0, \quad=0 \text { if } n=0 \\
& \int_{0}^{2 \pi} \theta\left(\theta_{1}-\theta_{1}^{\prime}\right) \sin n \theta_{1}^{\prime} d \theta_{1}^{\prime}=-\frac{\pi}{n} \cos n \theta_{1} \text { if } n \not x 0,=0 \text { if } n=0 \\
& \int_{0}^{2 \pi} \theta^{2}\left(\theta_{1}-\theta_{1}^{\prime}\right) \cos n \theta_{1}^{\prime} d \theta_{1}^{\prime}=\frac{\pi}{n^{2}} \cos n \theta_{1} \text { if } n \neq 0, \quad=\frac{\pi^{3}}{6}, \text { if } n=0,
\end{aligned}
$$

the Fourier's series for the function $\omega$ is obtained. The term $\omega_{0}$ corresponding to current sheets may be written

$$
\omega_{0}=\omega_{11}+\frac{p_{1} p_{2}}{a_{1} a_{2}} \omega_{00}
$$

where the principal part of $\omega$ is $\omega_{11}$ due to the angular component of current in the sheets. The term

$$
\frac{p_{1} p_{2}}{a_{1} a_{2}} \omega_{00}
$$

is due to the axial components of current in the sheets and is an infinitesimal of second order.

The functions $\omega_{11}$ and $\omega_{00}$ are special cases of the function $\omega_{n_{1} n_{2}}$ defined by

so that

$$
\omega_{n_{1}, n_{2}}\left(x, r, a_{1}, a_{2}\right) \equiv 4 \pi^{2} \sqrt{a_{1} a_{2}} \int_{0}^{x} d x^{\prime \prime} \int_{0}^{x^{\prime \prime}} d x^{\prime} \phi_{n_{1}, n_{2}}\left(x^{\prime}, r, a_{1}, a_{2}\right),
$$

$$
\omega^{\prime}{ }_{n_{1}, n_{2}}\left(x, r, a_{1}, a_{2}\right)=4 \pi^{2} \sqrt{a_{1} a_{2}} \int_{0}^{x} d x^{\prime} \phi_{n_{1}, n_{2}}\left(x^{\prime}, r, a_{1}, a_{2}\right) .
$$

The first-order infinitesimal $\omega_{1}$ given by eq 31 is found to be

$$
\begin{aligned}
\omega_{1}\left(x, \theta_{1}, \theta_{2}\right) & =-p_{1} \sum_{n=1}^{\infty} \frac{\sin n \theta_{1}}{n}\left[\omega^{\prime}{ }_{n+1,1}\left(x, r, a_{1}, a_{2}\right)+\omega_{n-1,-1}^{\prime}\left(x, r, a_{1}, a_{2}\right)\right] \\
& -p_{2} \sum_{n=1}^{\infty} \frac{\sin n\left(\pi-\theta_{2}\right)}{n}\left[\omega_{n+1,1}^{\prime}{ }_{n+1}\left(x, r, a_{2}, a_{1}\right)+\omega_{n-1}^{\prime}{ }_{n-1}\left(x, r, a_{2}, a_{1}\right)\right],
\end{aligned}
$$


and the second-order term $\omega_{2}$, derived from eq 32 becomes

$$
\begin{gathered}
\omega_{2}\left(x, \theta_{1}, \theta_{2}\right) \\
=4 \pi^{2} \sqrt{a_{1} a_{2}}\left\{p _ { 1 } ^ { 2 } \sum _ { n = 1 } ^ { \infty } \frac { \operatorname { c o s } n \theta _ { 1 } } { n _ { 2 } } \left[\phi_{n+1,1}\left(x, r, a_{1}, a_{2}\right)+\phi_{n-1},-1\right.\right. \\
+p_{2}^{2} \sum_{n=1}^{\infty} \frac{\left.\left.\cos r, a_{1}, a_{2}\right)\right]}{n\left(\pi-\theta_{2}\right)}\left[\phi_{n+1,1}\left(x, r, a_{2}, a_{1}\right)+\phi_{n-1,-1}\left(x, r, a_{2}, a_{1}\right)\right] \\
+p_{1} p_{2} \sum_{n_{1}=1}^{\infty} \sum_{n_{3}=1}^{\infty} \frac{1}{n_{1} n_{2}}\left[\left(\phi_{n_{1}+1,-n_{2}+1}+\phi_{n_{1}-1,-n_{2}-1}\right) \cos \left(n_{1} \theta_{1}+n_{2} \theta_{2}\right)\right. \\
\left.\left.-\left(\phi_{n_{1}+1, n_{2}+1}+\phi_{n_{1}-1, n_{2}-1}\right) \cos \left(n_{1} \theta_{1}-n_{2} \theta_{2}\right)\right]\right\}
\end{gathered}
$$

where the arguments of all the $\phi$-functions in the double series are $\left(x, r, a_{1}, a_{2}\right)$, respectively. In deriving these expressions from the expansion (eq 55), we have made use of the fact (shown by eq $57 \mathrm{~b}$ ) that $\phi_{-n_{1}, n_{3}} \equiv \phi_{n_{1}, n_{2}}$ and also of the relation (shown by eq $57 \mathrm{a}$ that

$$
\left.\phi_{n_{1}, n_{2}}\left(x, r, a_{1}, a_{2}\right)=\phi_{n_{2}, n_{1}} x, r, a_{2}, a_{1}\right),
$$

which obviously hold for the functions $\omega_{n_{1} n_{2}}$ and $\omega_{n_{1} n_{2}}^{\prime}$.

The approximate expressions for $\theta$ and $\theta^{2}$ given in eq 35 merely indicate that $\omega_{1}$ is found from eq 61 by retaining only the first two terms of each series and inserting the factor $\pi^{2} / 9$, while the second-order term, $\omega_{2}$, is sufficiently represented by merely extending the $n_{1}$, and $n_{2}$ summations each from 1 to 2 in the series given in eq 62 .

In the case of coaxial helices $(r=0)$, eq $57 \mathrm{a}$, shows that

$$
\phi_{n_{1} n_{2}}\left(x, 0, a_{1}, a_{2}\right)=\phi_{n_{1}}\left(x, a_{1}, a_{2}\right) \delta_{n_{1} n_{3}}=\phi_{n_{1}}(k) \delta_{n_{1} n_{3}},
$$

where

$$
k^{2}=\frac{4 a_{1} a_{2}}{x^{2}+\left(a_{1}+a_{2}\right)^{2}}
$$

and $\delta_{n_{1}, n_{2}}=1$, if $n_{1}=n_{2}$,=zero otherwise. Equation 61 shows that $\omega_{1}=0$, and eq 62 shows that

$$
\omega_{2} \rightarrow-4 \pi^{2} p_{1} p_{2} \sqrt{a_{1} a_{2}} \sum_{n=1}^{\infty}\left[\phi_{n-1}(k)+\phi_{n+1}(k)\right] \frac{\cos n\left(\theta_{1}-\theta_{2}\right),}{n^{2}}
$$

where $\theta_{1}-\theta_{2}$ is the azimuthal difference of the two terminals.

Hence the surviving terms are equivalent to those previously obtained for the coaxial case in eq 49 of the paper quoted (footnote 2 ). The terms $n_{1} n_{2} \omega_{11}(x), n_{1} n_{2} \omega_{00}(x), n_{1} n_{2} \omega_{2}\left(x, \theta_{1}-\theta_{2}\right)$ of this paper correspond to $\omega_{0}(x), \omega_{x}(x)$, and $\omega_{a}\left(x, \theta_{x}\right)\left(\theta_{x}=\theta_{1}-\theta_{2}\right)$ of that paper, where $n_{1} n_{2}$ $=1 / 4 \pi^{2} p_{1} p_{2}$.

For the coaxial helices in a current balance, the term $\omega_{2}$ compared with $\omega_{0}$ was found to be 10 parts in a million.

To derive a formula by which the mutual inductance of the two helices may be computed in the general case, where their axes are merely parallel, it is necessary to obtain expressions by which the functions $\phi_{n_{1} n_{3}}$ and their first and second partial integrals with respect to $x$ may be computed. None of the integrals, eq $57 \mathrm{a}, 57 \mathrm{~b}, 59$, and 60 , may be integrated in finite terms, so we are driven to expansions 
in series. Such expansions in oblate spheroidal harmonics are obtained in the following sections.

By reference to the relation (eq 63) it is evident that $\phi_{n_{1}, n_{2}}$, where $n_{1}$ and $n_{2}$ are any integers (positive, negative, or zero), is obtainable (by interchange of the arguments, $a_{1}$ and $a_{2}$ when necessary) from one of the following three irreducible cases:

$$
\begin{aligned}
& \text { Case } A ; n_{2}=n_{1} \geqslant 0 \\
& \text { Case } B ; 0 \leqslant n_{2}<n_{1} \\
& \text { Case } C ; n_{2}<0 \text { and } n_{1}>0
\end{aligned}
$$

However, this requires that we obtain expansions for $\phi_{n_{1} n_{2}}$ in these three cases, without committing ourselves to a restriction that $a_{2}<a_{1}$, that is, one that is valid when $a_{1}$ and $a_{2}$ are interchanged.

\section{PARTIAL DIFFERENTIAL EQUATIONS AND BOUNDARY CONDITIONS SATISFIED BY THE FUNCTION $\phi_{n_{1}, n_{3}}$}

From the differential equation satisfied by Bessel's function, combined with the integral representation of $\phi_{n_{1}, n_{2}}$ in (eq 57a), it is found that $\phi_{n_{1}, n_{2}}$ satisfies the two partial differential equations

$$
\left\{D_{x}^{2}+D_{r}^{2}+\frac{1}{r} D_{r}-\frac{n^{2}}{r^{2}}\right\} \frac{\phi_{n_{1}, n_{2}}}{\sqrt{a_{1} a_{2}}}=0 \text {, where } n=n_{1}-n_{2} \geqslant 0,
$$

and

$$
\left\{D_{x}^{2}+D_{a_{1}}^{2}+\frac{1}{a_{1}} D_{a_{1}}-\frac{n_{1}^{2}}{a_{1}^{2}}\right\} \frac{\phi_{n_{1}, n_{2}}}{\sqrt{a_{1} a_{2}}}=0,
$$

and also a similar equation, where $a_{1}$ and $n_{1}$ are replaced by $a_{2}$ and $n_{2}$. As to boundary conditions, it is evident that $\frac{\phi_{n_{1}, n_{2}}}{\sqrt{a_{1} a_{2}}}$ vanishes when $\sqrt{x^{2}+r^{2}} \rightarrow \infty$, or when $\sqrt{x^{2}+a_{1}^{2}} \rightarrow \infty$. The function is also regular in the neighborhood of $r=0$ or $\left(a_{1}=0\right)$ and is finite, continuous, and singlevalued in general and an even function of $x$.

The partial derivative with respect to $x, \phi_{n_{1}, n_{2}}^{\prime}$ is an odd function of $x$. Defining $\sigma$ by

$$
\begin{aligned}
\sigma & \equiv-\frac{\phi_{n_{1}, n_{2}}^{\prime}\left(+0, r, a_{1}, a_{2}\right)}{2 \pi \sqrt{a_{1} a_{2}}}=+\frac{\phi_{n_{1}}^{\prime}, n_{2}}{2 \pi \sqrt{a_{1} a_{2}}}\left(-0, r, a_{1}, a_{2}\right) \\
& =\frac{1}{2 \pi} \int_{0}^{\infty} t J_{n_{1}-n_{2}}(r t) J_{n_{1}}\left(a_{1} t\right) J_{n_{2}}\left(a_{2} t\right) d t,
\end{aligned}
$$

it will be seen that $\sigma$ must be zero when $r<\left|a_{1}-a_{2}\right|$ and when $\left.r\right\rangle a_{1}+a_{2}$, for the integral representation (eq 57b) exhibits $\phi_{n_{1}, n_{2}}$ as a double line integral around two parallel circles of radii $a_{1}$ and $a_{2}, x$ being the distance between their planes and $r$ the distance between their axes. In the case $n_{1}=n_{2}=1$, the function $\phi_{11}$ multiplied by $4 \pi^{2} \sqrt{a_{1} a_{2}}$ is the mutual inductance of the two parallel circles. 
Equation 57 b may be written

$$
\begin{aligned}
4 \pi^{2} \sqrt{a_{1} a_{2}} \phi_{n_{1}, n_{2}}(x, r) & =\int_{0}^{2 \pi} a_{2} \cos n_{2} \theta_{2} d \theta_{2} \int_{0}^{2 \pi} \frac{a_{1} \cos n_{1} \theta_{1} d \theta_{1}}{R_{12}} \\
& +\int_{0}^{2 \pi} a_{2} \sin n_{2} \theta_{2} d \theta_{2} \int_{0}^{2 \pi} \frac{a_{1} \sin n_{1} \theta_{1}}{R_{12}} d \theta_{1} .
\end{aligned}
$$

Each of these integrals is a special case of double-line integrals taken around the two circles of type

where

$$
\phi(x)=\int f_{2}\left(\theta_{2}\right) d s_{2} \int \frac{f_{1}\left(\theta_{1}\right) d s_{1}}{R_{12}}
$$

$$
d s_{1}=a_{1} d \theta_{1} \text { and } d s_{2}=a_{2} d \theta_{2}
$$

are elements of arc of the circles, $R_{12}$ being the distance between the two points of integration, $P_{1}$ in the first circle and $P_{2}$ on the second. The first of radius $a_{1}$ may be considered in the plane $x=0$ coaxial with the $x$-axis, the second parallel to it has its center at a point whose cylindrical coordinates are $(x, r, \theta)$. Since $1 / R_{12}$ is so fundamental in the theory of electrostatics, and because electrostatic problems are familiar to most of us, some guidance may be obtained by availing ourselves occasionally of an electrostatic interpretation of integrals involving the factor $1 / R_{12}$ in the integrand. Thus the first line integral in eq 68 , which is

$$
V=\int \frac{f_{1}\left(\theta_{1}\right) d s_{1}}{R_{12}}
$$

is the electrostatic potential at any point $P_{2}$ on the second circle when the first circle is a line charge with linear density $f_{1}\left(\theta_{1}\right)$. Hence, if the second circle is a line charge of linear density $f_{2}\left(\theta_{2}\right)$, then

$$
-\phi^{\prime}(x)=-D_{x} \phi=\int_{2} f_{2}\left(\theta_{2}\right) E_{x} d s_{2}, \text { where } E_{x}=-D_{x} V
$$

the $x$-component of the electric field of the first circle at any point $P_{2}$ of the second. Consequently, $-\phi^{\prime}=F_{x}=$ the $x$-component of the force which the first circle exerts upon the second. Clearly this vanishes when $x=0$ if the circles do not touch or intersect in this coplanar position, that is,

$$
\begin{aligned}
\phi^{\prime}(0) & =0 \text { if } 0<r<\left|a_{1}-a_{2}\right| \\
& =0 \text { if } a_{1}+a_{2}<r<\infty .
\end{aligned}
$$

In the remaining case, $\left|a_{1}-a_{2}\right|<r<a_{1}+a_{2}$, the circles intersect if they are brought into the same plane while keeping $r$ constant. This is the case for which figure 3 is drawn, assuming $a_{2}<a_{1}$ (but the argument may be made by reference to that figure, keeping in mind the other possibility, $a_{1}<a_{2}$ ). In this case the values of $\theta_{1}$ and $\theta_{2}$ corresponding to one point of intersection $P_{0}$ are called $\theta_{1}^{0}$ and $\theta_{2}^{0}$, respec- 
Equation 14 may therefore be written

$$
\begin{gathered}
\frac{a}{\sqrt{a^{2}+p^{2}}} \int_{0}^{s} f(x, \theta) d s=\frac{1}{2 \pi p}\left(\iint_{s} f(x, \theta) d S+\right. \\
+2 a \int_{0}^{2 \pi} d \theta\left\{p \theta\left(\theta_{e}-\theta\right) f\left(x_{e}, \theta\right)+\left[p^{2} \theta^{2}\left(\theta_{e}-\theta\right)-\frac{\pi^{2} p^{2}}{12}\right] f^{\prime}\left(x_{e}, \theta\right)\right\} \\
\left.-2 a \int_{0}^{2 \pi} d \theta\left\{p \theta\left(\theta_{i}-\theta\right) f\left(x_{i}, \theta\right)+\left[p^{2} \theta^{2}\left(\theta_{i}-\theta\right)-\frac{\pi^{2} p^{2}}{12}\right] f^{\prime}\left(x_{i}, \theta\right)\right\}\right),
\end{gathered}
$$

where $\bar{\theta}_{e}$ has been replaced by $\theta_{e}$, the distinction between the two being now no longer material, since $\theta_{e}$ appears only in $\theta\left(\theta_{e}-\theta\right)$, which is a periodic function of its argument with period $2 \pi$.

The parenthesis on the right of eq 16 , which has the factor $1 / 2 \pi p$, contains three orders of magnitudes. The first, or finite term, is the surface integral of $f$ taken over the sheet $S$.

If eq 16 be divided by $2 \pi N a$, the first member becomes $1 / s \int_{0}^{s} f d s=$ the line average of $f$ taken along the helix. The principal term on the right becomes $1 / S \mathcal{S S} f d S$, which is the surface average of $f$ taken over $S$. Equation 16 is therefore the required relation giving the difference of these two averages as a relatively small quantity in which two orders of magnitude have been retained.

By two successive applications of the transformation represented in eq 16 we may convert the two successive line integrals along the two helices (which represent their mutual inductance, $M$ ) into two successive surface integrals over their equivalent sheets plus small correction terms. To begin this, eq 9 may be written

$$
M=\frac{a_{2}}{\sqrt{a_{2}^{2}+p_{2}^{2}}} \int_{0}^{s_{2}} d s_{2} f\left(x_{2}, \theta_{2}\right)
$$

the integration being along the second helix,

$$
f\left(x_{2}, \theta_{2}\right)=\frac{a_{1}}{\sqrt{a_{1}^{2}+p_{1}^{2}}} \int_{0}^{s_{1}} d s_{1} F\left(x_{2}-x_{1}, \theta_{1}, \theta_{2}\right),
$$

the latter integration being taken along the first helix.

$$
\begin{aligned}
& \text { By use of eq } 16 \\
& \qquad f\left(x_{2}, \theta_{2}\right)=\frac{1}{2 \pi p_{1}}\left(\iint_{s_{1}} d S_{1} F\left(x_{2}-x_{1}, \theta_{1}, \theta_{2}\right)\right. \\
& +2 a_{1} \int_{0}^{2 \pi} d \theta_{1}\left\{p_{1} \Theta\left(\theta_{e_{1}}-\theta_{1}\right) F\left(x_{2}-x_{e_{1}}, \theta_{1}, \theta_{2}\right)\right. \\
& \left.-\left[p_{1}^{2} \theta^{2}\left(\theta_{e_{1}}-\theta_{1}\right)-\frac{\pi^{2} p_{1}^{2}}{12}\right] F^{\prime}\left(x_{2}-x_{e_{1}}, \theta_{1}, \theta_{2}\right)\right\} \\
& -2 a_{1} \int_{0}^{2 \pi} d \theta_{1}\left\{p_{1} \Theta\left(\theta_{i_{1}}-\theta_{1}\right) F\left(x_{2}-x_{i_{1}}, \theta_{1}, \theta_{2}\right)\right. \\
& \left.\left.-\left[p_{1}^{2} \theta^{2}\left(\theta_{i_{1}}-\theta_{1}\right)-\frac{\pi^{2} p_{1}^{2}}{12}\right] F^{\prime}\left(x_{2}-x_{i_{1}}, \theta_{1}, \theta_{2}\right)\right\}\right)
\end{aligned}
$$


tively. The angle $2 \psi$ between the intersecting ares may be taken as the angle $O_{1} P_{0} O_{2}$, so that

$\left\{\begin{array}{l}2 \psi=\theta_{2}^{0}-\theta_{1}^{0} \text {, where } 0 \leqslant \theta_{1}^{0} \leqslant \pi \text { and } 0 \leqslant \theta_{2}^{0} \leqslant \pi, \\ \text { so that } 0 \leqslant 2 \psi \leqslant \pi\end{array}\right\}$

The coordinates, $\theta_{1}^{0}=2 \pi-\theta_{1}^{0}$ and $\theta_{2}^{\prime 0}=2 \pi-\theta_{2}^{0}$ refer to the other point of intersection $P_{0}^{\prime}$.

When the two circles approach the same plane $(x \rightarrow+0)$, the infinitesimal ares in the neighborhood of $P_{0}$ (or $P_{0}^{\prime}$ ) contribute a finite amount to their mutual repulsion $F_{x}$ in the $x$-direction, because $\mathrm{R}_{12}$ vanishes at $P_{0}$ (and $P_{0}^{\prime}$ ). Arcs at a finite distance from these points contribute a vanishing amount to $F_{x}$. The curvature of the intersecting arcs becomes irrelevant, but of course the angle $2 \psi$ at which they intersect is important. The value of the integral may be computed as in the case of two endless straight-line charges approaching intersection, their linear charge densities being variable, but only the values of these densities at $P_{0}$ (or $P^{\prime}$ ) being effective. If we allow for discontinuities in the functions $f_{1}\left(\theta_{1}\right)$ and $f_{2}\left(\theta_{2}\right)$ (as for example in eq 31 and eq 32 , in the integrals defining $\omega_{1}$ and $\left.\omega_{2}\right)$, we find, when $\left|a_{1}-a_{2}\right|<r<a_{1}+a_{2}$ and $\phi$, defined by eq 68 , that

$$
\begin{aligned}
-\phi^{\prime}(+0)=\frac{2 \pi}{\sin 2 \psi}\left\{\left(\frac{f_{1}\left(\theta_{1}^{0}+0\right)+f_{1}\left(\theta_{1}^{0}-0\right)}{2}\right)\left(\frac{f_{2}\left(\theta_{2}^{0}+0\right)+f_{2}\left(\theta_{2}^{0}-0\right)}{2}\right)\right. \\
+\left(\frac{f_{1}\left(\theta_{1}^{\prime}+0\right)+f_{1}\left(\theta_{2}^{\prime 0}-0\right)}{2}\right)\left(\frac{f_{2}\left(\theta_{2}^{\prime 0}+0\right)+f_{2}\left(\theta_{2}^{\prime}-0\right)}{2}\right) \\
+\left(\frac{\pi-4 \psi}{\pi}\right)\left[\left(\frac{f_{1}\left(\theta_{1}^{0}+0\right)-f_{1}\left(\theta_{1}^{0}-0\right)}{2}\right)\left(\frac{f_{2}\left(\theta_{2}^{0}+0\right)-f_{2}\left(\theta_{2}^{0}-0\right)}{2}\right)\right. \\
\left.\left.+\left(\frac{f_{1}\left(\theta_{1}^{\prime 0}+0\right)-f_{1}\left(\theta_{1}^{\prime 0}-0\right)}{2}\right)\left(\frac{f_{2}\left(\theta_{2}^{\prime 0}+0\right)-f_{2}\left(\theta_{2}^{\prime 0}-0\right)}{2}\right)\right]\right\}
\end{aligned}
$$

For the case where $f_{1}$ and $f_{2}$ are continuous functions, this becomes

$$
-\phi^{\prime}(+0)=\frac{2 \pi}{\sin 2 \psi}\left[f_{1}\left(\theta_{1}^{0}\right) f_{2}\left(\theta_{2}^{0}\right)+f_{1}\left(\theta_{2}^{\prime 0}\right) f_{2}\left(\theta_{2}^{\prime 0}\right)\right]
$$

Using this in eq 67 leads to the following expression for the $\sigma$ defined in eq 66:

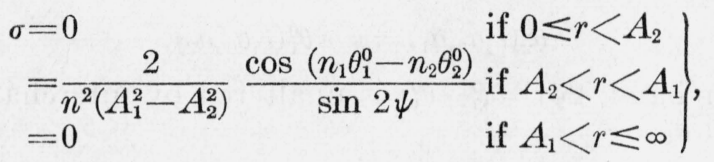

where

$$
\left.\begin{array}{l}
A_{1} \equiv a_{1}+a_{2} \mid \\
A_{2} \equiv\left|a_{1}-a_{2}\right|
\end{array}\right\} \text {, so that } 0<A_{2}<A_{1} .
$$

The absolute value $\left|a_{1}-a_{2}\right|$ is used for $A_{2}$, so that we need not commit ourselves on the question as to which of the two circular radii, $a_{1}$ or $a_{2}$, is the larger. 
The second member of eq 72 is a function of $r$, since $\theta_{1}^{0}$ and $\theta_{2}^{0}$ and $2 \psi=\theta_{2}^{0}-\theta_{1}^{0}$ are functions of $r$. However, it is more convenient to reverse this and to express $\sigma$ and $r$ as functions of $\psi$, which may be done by expressing $\theta_{1}^{0}$ as functions of $\psi$.

To do this we define $\xi_{0}^{\prime}$ by

$$
\begin{aligned}
& \xi_{0}^{\prime}=\frac{a_{1}-a_{2}}{a_{1}+a_{2}} \\
& \left.\xi_{0}=\sqrt{1-\xi_{0}^{2}}=\frac{2 \sqrt{a_{1} a_{2}}}{a_{1}+a_{2}}=\sqrt{1-\left(\frac{A_{2}}{A_{1}}\right)^{2}} \mid \begin{array}{l}
-1<\xi_{0}^{\prime}<1 \\
0<\xi_{0}<1
\end{array}\right\} .
\end{aligned}
$$

Considering the triangle $O_{1} P_{0} O_{2}$ of figure 3 , it is evident that

$$
\begin{gathered}
r=\left(a_{1}^{2}-2 a_{1} a_{2} \cos 2 \psi+a_{2}^{2}\right)^{\frac{1}{2}}=A_{1}\left(1-\xi_{0}^{2} \cos ^{2} \psi\right)^{\frac{1}{2}} \\
=a_{1}\left(1-\frac{a_{2}}{a_{1}} e^{2 i \psi}\right)^{\frac{1}{2}}\left(1-\frac{a_{2}}{a_{1}} e^{-2 i \psi}\right)^{\frac{1}{2}} \\
\sin 2 \psi=\frac{2}{A_{1}^{2}-A_{2}^{2}} \sqrt{\left(A_{1}^{2}-r^{2}\right)\left(r^{2}-A_{2}^{2}\right)} \\
\cos \theta_{1}^{0}=\frac{a_{1}-a_{2} \cos 2 \psi}{r} \mid \text { and }\left\{\begin{array}{l}
\cos \theta_{2}^{0}=\frac{a_{1} \cos 2 \psi-a_{2}}{r} \\
\sin \theta_{2}^{0}=\frac{a_{1} \sin 2 \psi}{r}
\end{array}\right\} . \\
\left.\sin \theta_{1}^{0}=\frac{a_{2} \sin 2 \psi}{r}\right\} \\
e^{i \theta_{1}^{\circ}=} \frac{a_{1}-a_{2} e^{-2 i \psi}}{r}=\left(\frac{a_{1}-a_{2} e^{-2 i \psi}}{a_{1}-a_{2} e^{2 i \psi}}\right)^{\frac{1}{2}}=\frac{1+\xi_{0}^{\prime}}{2} \frac{1-\xi_{0}^{\prime}}{1+\xi_{0}^{\prime}} e^{-2 i \psi} \\
e^{i \theta_{2}^{\circ}}=\frac{a_{1} e^{2 i \psi}-a_{2}}{r}=e^{i\left(\theta_{1}^{\circ}+2 \psi\right)} .
\end{gathered}
$$

From these relations it follows that

When considering $\theta_{1}^{0}$ and $\theta_{2}^{0}$ as functions of $r, a_{1}$, and $a_{2}$, this may be indicated by writing $\theta_{1}^{0}=\theta_{1}^{0}\left(r, a_{1}, a_{2}\right)$ and $\theta_{2}^{0}=\theta_{2}^{0}\left(r, a_{1}, a_{2}\right)$. It is evident from these definitions that when we interchange the arguments $a_{1}$ and $a_{2}, \theta_{1}^{0}$ goes into $\pi-\theta_{2}^{0}$ and $\theta_{2}^{0}$ goes into $\pi-\theta_{1}^{0}$, that is,

and

$$
\theta_{\mathbf{1}}^{0}\left(r, a_{2}, a_{1}\right)=\pi-\theta_{2}^{0}\left(r, a_{1}, a_{2}\right)
$$

$$
\theta_{2}^{0}\left(r, a_{2}, a_{1}\right)=\pi-\theta_{1}^{0}\left(r, a_{1}, a_{2}\right) .
$$

On the other hand, $2 \psi\left(=\theta_{2}^{0}-\theta_{1}^{0}\right)$ is unaltered by interchange of arguments $a_{1}$ and $a_{2}$. 
By eq 77 we may write (if $\mathscr{R}(\quad)$ denotes the real part)

$$
\begin{aligned}
& \cos \left(n_{1} \theta_{1}^{0}-n_{2} \theta_{2}^{0}\right)=\mathscr{R}\left(\frac{1+\xi_{0}^{\prime}}{2}\right)^{n_{1}-n_{2} e^{-2 i n_{2} \psi}\left[1-\frac{1-\xi_{0}^{\prime}}{1+\xi_{0}^{\prime}} e^{-2 i \psi}\right]^{n_{1}-n_{3}}} \\
& =\frac{\left(\frac{1+\xi_{0}^{\prime}}{2}\right)^{n_{1}-n_{2}}}{\left(1-\xi_{0}^{2} \cos ^{2} \psi\right)^{\frac{n_{1}-n_{2}}{2}} \Gamma\left(n_{1}-n_{2}+1\right) .} \\
& \cdot \sum_{k=0}^{n_{1}-n_{2}}(-1)^{k}\left(\frac{1-\xi_{0}^{\prime}}{1+\xi_{0}^{\prime}}\right)^{k} \frac{\cos 2\left(k+n_{2}\right) \psi}{\Gamma(k+1) \Gamma\left(1+n_{1}-n_{2}-k\right)} \\
& =\frac{(-1)^{n_{2}}\left(\frac{a_{1}}{A_{1}}\right)^{n_{1}}\left(\frac{a_{2}}{A_{1}}\right)^{-n_{2}} \Gamma\left(n_{1}-n_{2}+1\right)}{\left(1-\xi_{0}^{2} \cos ^{2} \psi\right)^{\frac{n_{1}-n_{2}}{2}}} \text {. } \\
& \cdot \sum_{k=n_{2}}^{n_{1}} \frac{(-1)^{k}\left(\frac{a_{2}}{a_{1}}\right)^{k} \cos 2 k \psi}{\left(1+n_{1}-k\right) \Gamma\left(1-n_{2}+k\right) .}
\end{aligned}
$$

Hence, if we define the integer $n$ by

$$
n=n_{1}-n_{2} \text {, so that } n \geqq 0 \text { in all cases, } A, B \text {, and } C \text {, }
$$

while $n_{2}$ may be positive or negative, then

$$
\begin{aligned}
& \sigma=0 \ldots \ldots \ldots-\ldots \text { when } 0 ₹ r<A_{2} \\
& =\frac{2}{\xi_{2}^{2} \pi^{2} A^{2} \sin 2 \psi}\left(\frac{1+\xi_{0}^{\prime}}{2}\right)^{n} \frac{\Gamma(n+1)}{\left(1-\xi_{0}^{2} \cos ^{2} \psi\right)^{\frac{n}{2}}} . \\
& \cdot \sum^{n}(-1)^{k}\left(\frac{1-\xi_{0}^{\prime}}{1+\xi_{0}^{\prime}}\right)^{R} \frac{\cos 2\left(k+n_{2}\right) \psi}{\Gamma(k+1) \Gamma(1+n-k)} \\
& \text { when } A_{2}<r<A_{1} \\
& =0 \ldots \ldots \ldots-\ldots \text { when } A_{1}<r \overline{<} \infty
\end{aligned}
$$

In case $n=0$, this series reduces to the simple term $\cos 2 n_{2} \psi$, so tha

$$
\sigma_{m, m}=\frac{2 \cos 2 m \psi}{\pi^{2}\left(A_{1}^{2}-A_{2}^{2}\right) \sin 2 \psi} .
$$

Quite a different electrostatic interpretation of $\phi_{n_{1 n_{2}}}$ will now be useful. If $x, r$, and $\theta$ are considered as the cylindrical coordinates of a point $P$ in space, the function $V(x, r, \theta)=\left(a_{1} a_{2}\right)^{-\frac{1}{2}} \phi_{n 1}, n 2\left(x, r, a_{1}, a_{2}\right)$ $\cos \left(n_{1}-n_{2}\right) \theta$ is the Newtonian potential at any point $P$ due to a simple distribution of charge upon the annular surface $\left(A_{2}<r<A_{1}, x=0\right)$, whose surface density is $\sigma \cos \left(n_{1}-n_{2}\right) \theta$, so that the $\sigma$ defined by eq 66 or 80 plays the same part for solutions of eq 64 that surface density on the annulus plays for solutions of Laplace's equation.

In the following section an expansion for $\phi_{n_{1}, n_{2}}$ is obtained in terms of the normal functions of oblate spheroidal coordinates, these being 
used because in terms of them $\phi_{n_{1}}, n_{2}$ admits of a single expansion valid for all real values of $x$ and for all positive or zero values of $r, a_{1}$, and $a_{2}$, whereas ordinary spherical coordinates require three different expansions according as $0 \leqslant r \leqslant A_{2}, A_{2} \leqslant r \leqslant A_{1}$, or $A_{1} \leqslant r \leqslant \infty$, and the expansion in the second of these cases is somewhat complicated. In this respect, toroidal coordinates are superior to spherical but inferior to spheroidal, as the expansions require only two cases.

\section{EXPANSION OF $\phi_{n_{1} n_{2}}$ IN OBLATE SPHEROIDAL COORDINATES}

Instead of the cylindrical coordinates $(x, r, \theta)$ the (oblate) spheroidal coordinates, $\xi, \eta, \theta$ (the same $\theta$ ), may be used to designate a point in space, where

$$
\left.\begin{array}{l}
x=A_{1} \xi \eta \\
r=A_{1} \sqrt{\left(1-\xi^{2}\right)\left(1+\eta^{2}\right)}
\end{array}\right\}, \text { where }\left\{\begin{array}{l}
-1 \leqslant \xi \leqslant 1 \\
0 \leqslant \eta \leqslant \infty
\end{array}\right\} .
$$

Solving for $\xi$ ad $\eta$ gives

$$
\left.\begin{array}{l}
\xi^{2}=\frac{1}{2 A_{1}^{2}}\left\{A_{2}^{1}-x^{2}-r^{2}+\sqrt{\left[x^{2}+\left(r+A_{1}\right)^{2}\right]\left[x^{2}+\left(r-A_{1}\right)^{2}\right]}\right\} \\
\left.\eta^{2}=\frac{1}{2 A_{1}^{2}}\left\{x^{2}+r^{2}-A_{1}^{2}+\sqrt{\left[x^{2}+\left(r+A_{1}\right)^{2}\right]\left[x^{2}+\left(r-A_{1}\right)^{2}\right]}\right]\right\}
\end{array}\right\}
$$

Also from eq 81, we find

$$
d x^{2}+d r^{2}=A_{1}^{2}\left(\xi^{2}+\eta^{2}\right)\left[\frac{d \xi^{2}}{1-\xi^{2}}+\frac{d \eta^{2}}{1+\eta^{2}}\right] .
$$

The locus of the equation, $\eta=$ constant, is the entire surface of the oblate spheroid, whose equation is

$$
x^{2} / A_{1}^{2} \eta^{2}+r^{2} / A_{1}^{2}\left(1+\eta^{2}\right)=1,
$$

whose foci are at $\left(x=0, r= \pm A_{1}\right)$. In particular, the locus of $\eta=0$ is both sides of the circular disk $\left(x=0,0 \leqslant r, \leqslant A_{1}\right)$, at the edge of which $\xi=0$, and $\xi$ increases from zero to 1 on the side $x=+0$ as the point passes from the edge to the origin, or $x$-axis, while $\xi$ decreases from 0 to -1 on the side $x=-0$ as the point moves down to the axis. The locus $\xi=+1$ is the positive $x$-axis, and $\xi=-1$ is the negative $x$-axis.

The hyperboloid which is confocal with every such spheroid whose equation is

$$
-x^{2} / A_{1}^{2} \xi^{2}+r^{2} / A_{1}^{2}\left(1-\xi^{2}\right)=1
$$

must be considered as the two loci, that half for which $x>0$ being the locus $\xi=\xi_{1}>0$, and that for which $x<0$ being the locus $\xi=-\xi_{1}<0$. Large values of $\eta$ correspond to points far away from the origin, since $1+\eta^{2}=\left(x^{2}+r^{2}\right) / A_{1}^{2}+\xi^{2}$. The differential equation (64) becomes

$$
\left\{D_{\xi}\left[\left(1-\xi^{2}\right) D_{\xi}\right]-\frac{n^{2}}{1-\xi^{2}}\right\} \frac{\phi_{n_{1}, n_{2}}}{\sqrt{a_{1} a_{2}}}=-\left\{D_{\eta}\left[\left(1+\eta^{2}\right) D_{\eta}\right]+\frac{n^{2}}{1+\eta^{2}}\right\} \frac{\phi_{n_{1}, n_{2}}}{\sqrt{a_{1} a_{2}}}
$$


which has solutions of the form

$$
\frac{\phi_{n_{1} n_{2}}}{\sqrt{a_{1} a_{2}}}=P(\xi) R(\eta)
$$

where $P(\xi)$ is a solution of

$$
D_{\xi}\left[\left(1-\xi^{2}\right) P^{\prime}\right]+\left[\mu(\mu+1)-\frac{n^{2}}{1-\xi^{2}}\right] P=0 \text {, where } P^{\prime}=D_{\xi} P
$$

and $R(\eta)$ is a solution of

$$
\begin{gathered}
D_{\eta}\left[\left(1+\eta^{2}\right) R^{\prime}\right]-\left[\mu(\mu+1)-\frac{\eta^{2}}{1+\eta^{2}}\right] R=0 \\
n \equiv n_{1}-n_{2} .
\end{gathered}
$$

As shown in the preceding section, it is sufficient to consider $n=n_{1}-n_{2}$, where $n$ is a positive integer, or zero, while $n_{2}$ is either positive, negative, or zero, and $n_{1} \geqslant 0$. Equation 87 goes into the same type as eq 86 in the independent variable $z$, where $\eta=i z$.

One solution of eq 86 for the case where $-1<\xi<1$ may be taken as $P=P_{\mu}^{n}(\xi)$, which is (Ferrer's) associated Legendre function. This reduces to the accepted value when $\nu=$ an integer, if we define it, for general values of $\mu, \nu$ when $|1-\xi|<2$ by

$$
P_{\mu}^{\nu}(\xi)=\frac{\left(1-\xi^{2}\right)^{\frac{\nu}{2}} \Gamma(\mu+\nu+1)}{2^{\nu} \Gamma(\mu-\nu+1) \Gamma(\nu+1)} F\left(\nu-\mu, \nu+\mu+1, \nu+1 ; \frac{1-\xi}{2}\right) .
$$

We make use of the two recurrence relations

$$
\begin{gathered}
(2 \mu+1) \xi P_{\mu}^{\nu}(\zeta)=(\mu-\nu+1) P_{\mu+1}^{\nu}(\zeta)+(\mu+\nu) P_{\mu-1}^{\nu}(\zeta) \\
(2 \mu+1)\left(1-\zeta^{2}\right) P_{\mu}^{\prime \nu}(\xi)=-(\mu-\nu+1) \mu P_{\mu+1}^{v}(\xi)+(\mu+\nu)(\mu+1) P_{\mu-1}^{\nu}(\xi) .
\end{gathered}
$$

Also when $n$ is a positive integer

$$
P_{\mu}^{n}(\xi)=\left(1-\xi^{2}\right)^{\frac{n}{2}} D_{\xi}^{n} P_{\mu}(\xi) .
$$

We require also the following expressions when $\mu=\nu+s$, where $s$ is a positive integer or zero.

$$
\begin{aligned}
& P_{\nu+s}^{\nu}(\xi)=\frac{\left(1-\xi^{2}\right)^{\frac{\nu}{2}} \Gamma(s+2 \nu+1)}{2^{\nu} \Gamma(s+1) \Gamma(\nu+1)} \\
& F\left(-s, s+2 \nu+1, \nu+1 ; \frac{1-\xi}{2}\right) \text {, if }|1-\xi|<2 \\
& P_{\nu+2 s}^{\nu}(\xi)=\frac{(-1)^{s} 2 \nu\left(1-\xi^{2}\right)^{\frac{\nu}{2}} \Gamma\left(s+\nu+\frac{1}{2}\right)}{\sqrt{\pi}} F\left(-s, s+\nu+\frac{1}{2}, \frac{1}{2} ; \xi^{2}\right)
\end{aligned}
$$


Also when $\left|1-\xi^{2}\right|<1$, there are the two relations:

$$
\begin{gathered}
P_{\nu+2 s}^{\nu}(\xi)=\frac{2^{\nu}\left(1-\xi^{2}\right)^{\frac{\nu}{2}} \Gamma(s+\nu+1) \Gamma\left(s+\nu+\frac{1}{2}\right)}{\Gamma(\nu+1) \Gamma(s+1) \Gamma\left(s+\frac{1}{2}\right)} . \\
F\left(-s, s+\nu+\frac{1}{2}, \nu+1 ; 1-\xi^{2}\right) \\
P_{\nu+2 s+1}^{\nu}(\xi)=\frac{2^{\nu}\left(1-\xi^{2}\right)^{\frac{\nu}{2}} \Gamma(s+\nu+1) \Gamma\left(s+\nu+\frac{3}{2}\right)}{\Gamma(\nu+1) \Gamma(s+1) \Gamma\left(s+\frac{3}{2}\right)} . \\
\xi F\left(-s, s+\nu+\frac{3}{2}, \nu+1 ; 1-\xi^{2}\right) .
\end{gathered}
$$

The hypergeometric functions in the last five expressions are polynomials, if $s$ is a positive integer or zero, but eq $93,95 \mathrm{a}$, and $95 \mathrm{~b}$ are valid if $\nu$ and $s$ are unrestricted.

The importance of the case $\mu=\nu+s$ lies in the fact that if $\nu$ is any given constant greater than -1 , the infinite set of functions $P_{\nu+8}^{\nu} \xi$ constitute a complete set of orthogonal functions for the range -1 $<\xi<1$, for

and

$$
\int_{-1}^{\prime} P_{\nu+s_{1}}^{\nu}(\xi) P_{\nu+s_{2}}^{\nu}(\xi) d \xi=0 \text { if } s_{1} \neq s_{2}
$$

$$
\int_{-1}^{\prime}\left[P_{\nu+s}^{\nu}(\xi)\right]^{2} d \xi=\frac{2 \Gamma(s+2 \nu+1)}{(2 s+2 \nu+1) \Gamma(s+1)} .
$$

For the range $0<\xi<1$ the set of even functions of $\xi, P_{\nu+28}^{\nu} \xi$, are a closed set of orthogonal functions, as also are the odd functions.

If a function is developable in terms of these normal functions, its development is

$$
\left.f(\xi)=\sum_{s=0}^{\infty}\left(s+\nu+\frac{1}{2}\right) \frac{\Gamma(s+1)}{\Gamma(s+2 \nu+1)} P_{\nu+s}^{\nu}(\xi) \int_{-1}^{\prime} f\left(\xi_{1}\right) P_{\nu+s}^{\nu} \xi_{1}\right) d \xi_{1} \text { for }-1<\xi<1 .
$$

A special case is

$$
\begin{aligned}
f(\xi)=2 \sum_{s=0}^{\infty}\left(2 s+\nu+\frac{1}{2}\right) \frac{\Gamma(2 s+1)}{\Gamma(2 s+2 \nu+1)} P_{\nu+2 s}^{\nu}(\xi) \\
\int_{0}^{\prime} f\left(\xi_{1}\right) P_{\nu+2 s}^{\nu}(\xi) d \xi_{1} \text { for } 0<\xi<1 .
\end{aligned}
$$

For the range here needed, $0 \leqslant \eta \leqslant \infty$, it is convenient to take the general solution of eq 87 in the form

$$
R(\eta)=C_{1} R_{\mu}^{n}(\eta)+C_{2} S_{\mu}^{n}(\eta),
$$


where

$$
\begin{aligned}
R_{\mu}^{\nu}(\eta) & \equiv \frac{\left(1+\eta^{2}\right)^{-\left(\frac{\mu+1}{2}\right)}}{2^{\mu}} \cdot \frac{\Gamma(\mu-\nu+1)}{\Gamma\left(\mu+\frac{3}{2}\right)} \cdot F\left(\frac{\mu+\nu+1}{2}, \frac{\mu-\nu+1}{2}, \mu+\frac{3}{2} ; \frac{1}{1+\eta^{2}}\right) \\
& =\frac{2 \Gamma(\mu-\nu+1) i^{\mu+1}}{\sqrt{\pi} \cos \nu \pi \Gamma(\mu+\nu+1)} Q_{\mu}^{\nu}(i \eta) \\
S_{\mu}^{\nu}(\eta) & =\frac{\left(1+\eta^{2}\right)^{\frac{\mu}{2}} 2^{\mu} \pi}{\Gamma(\mu-\nu+1) \Gamma\left(\frac{1}{2}-\mu\right)} F\left(-\frac{\mu}{2}+\frac{\nu}{2},-\frac{\mu}{2}-\frac{\nu}{2}, \frac{1}{2}-\mu ; \frac{1}{1+\eta^{2}}\right) \\
& =-\frac{\sin (\mu+\nu) \pi}{2} R_{-\mu-1}^{\nu}(\eta) .
\end{aligned}
$$

The relation

$$
\left(1+\eta^{2}\right)\left[R_{\mu}^{\nu} S_{\mu}^{\prime \nu}-R_{\mu}^{\prime \nu} S_{\mu}^{\nu}\right]=2 \cos \mu \pi \frac{\Gamma(\mu-\nu+1)}{\Gamma(\mu+\nu+1)}
$$

shows that these two functions are linearly independent when $\nu=n$ and $\mu=m \geqslant n$. The two recurrence relations

$$
\begin{gathered}
(2 \mu+1) \eta R_{\mu}^{\nu}=(\mu-\nu) R_{\mu-1}^{\nu}-(\mu+\nu+1) R_{\mu+1}^{\nu} \\
(2 \mu+1)\left(1+\eta^{2}\right) R_{\mu}^{\nu}=-(\mu-\nu)(\mu+1) R_{\mu-1}^{\nu}-(\mu+\nu+1) \mu R_{\mu+1}^{\nu}
\end{gathered}
$$

are also satisfied by the functions $S_{\mu}^{\nu}$.

Since $Q_{\mu}^{n}(z)=\left(z^{2}-1\right)^{\frac{n}{2}} D_{z}^{n} Q_{\mu}(z)$, the analogs of eq 92 are

$$
\begin{aligned}
& R_{\mu}^{n}(\eta)=\frac{(-1)^{n} \Gamma(\mu-n+1)}{\Gamma(\mu+n+1)}\left(1+\eta^{2}\right)^{\frac{n}{2}} D_{\eta}^{n} R_{\mu}(\eta) \\
& S_{\mu}^{n}(\eta)=\frac{\Gamma(\mu-n+1)}{\Gamma(\mu+n+1)}\left(1+\eta^{2}\right)^{\frac{n}{2}} D_{\eta}^{n} S_{\mu}(\eta) .
\end{aligned}
$$

By use of the recurrence relations, eq 90, 91, 101a, and 101b, we obtain equations of the form

$$
(2 \mu+1) P_{\mu}^{\nu}(\xi) R_{\mu}^{\nu}(\eta)=-A_{1} D_{x}\left[P_{\mu-1}^{\nu}(\xi) R_{\mu-1}^{\nu}(\eta)+P_{\mu+1}^{\nu}(\xi) R_{\mu+1}^{\nu}(\eta)\right]
$$

and three others obtained by replacing $R_{\mu}^{\nu}(\eta)$ by $S_{\mu}^{\nu}(\eta)$ and then $P_{\mu}^{\nu}(\xi)$ by $Q_{\mu}^{\nu}(\xi)$ in each of the two relations. It is evident from eq 99 
that $R_{\mu}^{\nu} \rightarrow 0$ when $\eta \rightarrow \infty$ if $\mu>-1$, while $S_{\mu}^{\nu} \rightarrow \infty$ if $\mu>0$. We also place here for reference

$$
\left.\begin{array}{c}
R_{\mu}^{\nu}(0)=\frac{\Gamma\left(\frac{\mu-\nu+1}{2}\right)}{2^{\nu} \Gamma\left(\frac{\mu+\nu}{2}+1\right)} \quad \text { and } \quad R_{\mu}^{\prime \nu}(0)=-\frac{\Gamma\left(\frac{\mu-\nu}{2}+1\right)}{2^{\nu-1} \Gamma\left(\frac{\mu+\nu+1}{2}\right)} \\
S_{\mu}^{\nu}(0)=\frac{(\cos \mu \pi+\cos \nu \pi)}{2^{\nu+1}} \frac{\Gamma\left(\frac{\mu-\nu+1}{2}\right)}{\Gamma\left(\frac{\mu+\nu}{2}+1\right)} \text { and } S_{\mu}^{\prime \nu}(0) \\
=\frac{(\cos \mu \pi-\cos \nu \pi)}{2^{\nu}} \frac{\Gamma\left(\frac{\mu-\nu}{2}+1\right)}{\Gamma\left(\frac{\mu+\nu+1}{2}\right)}
\end{array}\right\}
$$

The reciprocal distance from $P_{1}\left(\xi_{1}, \eta_{1}, \theta_{1}\right)$ to $P(\xi, \eta, \theta)$ has the known expansion

$\frac{1}{R_{P_{1} P}}=\frac{2}{A_{1}} \sum_{n=0}^{\infty} \epsilon_{n} \cos n\left(\theta-\theta_{1}\right) \sum_{m=n}^{\infty}(-1)^{m}\left(m+\frac{1}{2}\right) P_{m}^{n}(\xi) R_{m}^{n}(\eta) P_{m}^{n}\left(\xi_{1}\right) S_{m}^{n}\left(\eta_{1}\right),(10$

provided that $\eta>\eta_{1}$, that is, the point $P$ lies outside the ellipsoid, $\eta_{1}$, passing through $P_{1}$. When it is inside, $\eta<\eta_{1}$ and the above expansion is merely modified by interchanging the arguments $\eta$ and $\eta$. This expansion is very similar in form to that of spherical coordinates given in eq 46. A comparison of eq 105 with eq 47 gives the expansion in spheroidal coordinates when $\eta>\eta_{1}$

$$
\frac{\phi_{n}\left(x-x_{1}, r, r_{1}\right)}{\sqrt{r r_{1}}}=\frac{1}{A_{1}} \sum_{m=n}^{\infty}(-1)^{m}\left(m+\frac{1}{2}\right) P_{m}^{n}(\xi) R_{m}^{n}(\eta) P_{m}^{n}\left(\xi_{1}\right) S_{m}^{n}\left(\eta_{1}\right)
$$

with interchange of $\eta$ and $\eta_{1}$ in case $\eta<\eta^{1}$. This is similar to the expansion of the same function in spherical coordinates given in eq 48 .

Passing now to the case $\eta=0$, which corresponds to $x_{1}=0$ and $0 \leqslant r_{1}=A_{1}\left(1-\xi^{2}\right)^{1 / 2}<A_{1}$ so that the ellipsoid, $\eta_{1}$, shrinks into the circular disc, we find from eq 104 that

$$
S_{n+2 s+1}^{n}(0)=0 \text { and } S_{n+2 s}^{n}(0)=\frac{(-1)^{n}}{2^{n}} \frac{\Gamma\left(s+\frac{1}{2}\right)}{\Gamma(s+n+1)} .
$$

Hence if

$$
k^{2}=\frac{4 r r_{1}}{x^{2}+\left(r+r_{1}\right)^{2}}
$$

then

$$
\begin{gathered}
\frac{\phi_{n}\left(x, r, r_{1}\right)}{\sqrt{r r_{1}}}=\frac{\phi_{n}(k)}{\sqrt{r r_{1}}} \\
=\frac{1}{A_{1} 2^{n}} \sum_{s=0}^{\infty}\left(2 s+n+\frac{1}{2}\right) \frac{\Gamma\left(s+\frac{1}{2}\right)}{\Gamma(s+n+1)} P_{n+2 s}^{n}(\xi) R_{n+2 s}^{n}(\eta) P_{n+2 s}^{n}\left(\xi_{1}\right),
\end{gathered}
$$


which is valid for all values of $\eta$, as is also the integral representation

$$
\frac{\phi_{n}(k)}{\sqrt{r r_{1}}}=\int_{0}^{\infty} e^{-1 x 1 t} J_{n}(r t) J_{n}\left(r_{1} t\right) d t .
$$

If $U$ is a solution of eq 64 , which vanishes when $x^{2}+r^{2} \rightarrow \infty$ and such that

$$
\sigma \equiv-\frac{1}{2 \pi}\left[D_{x} U\right]_{x-+0}=+\frac{1}{2 \pi}\left[D_{x} U\right]_{x=-0}
$$

is a given function of $r_{1}\left(=A_{1}\left(1-\xi_{1}^{2}\right) \frac{1}{2}\right)$

on the circular annulus $x=0 A_{2}<r_{1}<A_{1}$, and $\sigma=0$ when $0 \leqslant r_{1}<A_{2}$, and when $r>A_{1}$, then $\xi_{0}$ being defined as in eq 74

$$
U=2 \pi \int_{A_{2}}^{A_{1}} \frac{r_{1} \sigma\left(r_{1}\right) \phi_{n}(k) d r_{1}}{\sqrt{r r_{1}}}=2 \pi A_{1}^{2} \int_{0}^{\xi_{0}} \frac{\xi_{1} \sigma\left(\xi_{1}\right) \phi_{n}(k)}{\sqrt{r r_{1}}} d \xi_{1} .
$$

Using eq 108 in this gives

$$
\begin{aligned}
U=\frac{2 \pi A_{1}}{2^{n}} \sum_{s=0}^{\infty}\left(2 s+n+\frac{1}{2}\right) & \frac{\Gamma\left(s+\frac{1}{2}\right)}{\Gamma(s+n+1)} \\
& P_{n+2 s}^{n}(\xi) \cdot R_{n+2 s}^{n}(\eta) . \int_{0}^{\xi_{0}} \xi_{1} \sigma\left(\xi_{1}\right) P_{n+2 s}^{n}\left(\xi_{1}\right) d \xi_{1} .
\end{aligned}
$$

To confirm this result we note that the function $U$ given by eq 111 is a solution of eq 64 , which vanishes when $x^{2}+r^{2} \longrightarrow \infty$.

Also $D_{x} U_{x= \pm 0}=0$ when $r>A_{1}$, since $\left(D_{x} U_{x=0}\right)=\left(\frac{1}{A_{1} \eta} D_{\xi} U\right)_{\xi=0}$ and $P_{n+28}^{\prime n}(0)=0$.

When $r<A_{1}, 2 \pi \sigma=-\left(D_{x} U\right)_{x=+0}=-\frac{1}{A_{1} \xi}\left(D_{\eta} U\right)_{\eta=0}$ and eq 111 gives, by use of eq 104 ,

$\xi \sigma(\xi)=2 \sum_{s=0}^{\infty}\left(2 \xi+n+\frac{1}{2}\right) \frac{\Gamma(2 s+1)}{\Gamma(2 s+2 n+1)} P_{n+2 s}^{n}(\xi) \cdot \int_{0}^{\xi_{0}} \xi_{1} \sigma\left(\xi_{1}\right) P_{n+2 s}^{n}\left(\xi_{1}\right) d \xi_{1}$

for $0<\xi<1$.

Reference to eq $98^{\prime}$ for the case $\nu=n$ shows that the second member of eq 112 is the development in normal functions of the function $\xi \sigma(\xi)$, which is zero when $\xi_{0}<\xi<1$, that is, when $0 \leqslant r<A_{2}$, which shows that eq 111 is the required solution.

The same confirmation results by using eq 109 in eq 110, which give

which gives

$$
U=2 \pi \int_{0}^{\infty} e^{-|x| t} J_{n}(r t) d t \int_{A_{3}}^{A_{1}} r_{1} \sigma\left(r_{1}\right) J_{n}\left(r_{1} t\right) d r_{1}
$$

$$
\sigma \equiv-\frac{1}{2 \pi}\left(D_{x} U\right)_{x=+0}=\int_{0}^{\infty} t J_{n}(r t) d t \int_{A_{3}}^{A_{1}} r_{1} \sigma\left(r_{1}\right) J_{n}\left(r_{1} t\right) d r_{1},
$$

which is Hankel's integral representation of a function of $r$, which is equal to $\sigma$ when $A_{2}<r<A_{1}$ and is zero when $0 \leqslant r<A_{2}$ and when $r>A_{1}$. 
The function

$$
\frac{\phi_{n_{1} n_{2}}}{\sqrt{a_{1} a_{2}}}
$$

is equal to $U$ if the density (eq 80 ) is used. It is then necessary to evaluate the integral

$$
\begin{aligned}
C_{s}^{n_{1}, n_{2} \equiv} \equiv & \int_{0}^{\xi_{0}} \xi_{1} \sigma\left(\xi_{1}\right) P_{n+2 s}^{n}\left(\xi_{1}\right) d \xi_{1}=\frac{1}{\pi^{2} A_{1}{ }^{2}}\left(\frac{1+\xi_{0}^{\prime}}{2}\right)^{n} \cdot \frac{2 \Gamma(n+1)}{\xi_{0}{ }^{2}} . \\
& \cdot \sum_{k=0}^{n} \frac{(-1)^{k}\left(\frac{1-\xi_{0}^{\prime}}{1+\xi_{0}^{\prime}}\right)^{k}}{\Gamma(k+1) \Gamma(1+n-k)} \int_{0}^{\xi_{0}} \frac{\xi_{1} \cos 2\left(k+n_{2}\right) \psi P_{n+2 s}^{n}\left(\xi_{1}\right) d \xi_{1}}{\left(1-\xi_{0}{ }^{2} \cos ^{2} \psi\right)_{2}^{n} \cdot \sin 2 \psi}
\end{aligned}
$$

Now by eq 75 and eq 81 , when $\eta=0$

$$
\frac{r_{1}}{A_{1}}=\left(1-\xi_{1}^{2}\right)^{\frac{1}{2}}=\left(1-\xi_{0}^{2} \cos ^{2} \psi\right)^{\frac{1}{2}} \text {, so that } \xi_{1}=\xi_{0} \cos \psi
$$

and

$$
\xi_{1} d \xi_{1}=-\frac{\xi_{0}{ }^{2}}{2} \sin 2 \psi d \psi=-\frac{r_{1} d r_{1}}{A_{1}{ }^{2}}
$$

so this becomes

$$
\begin{aligned}
C_{s}^{n_{1}, n_{2}=} \frac{\Gamma(n+1)}{\pi^{2} A_{1}^{2}}\left(\frac{1+\xi_{0}^{\prime}}{2}\right)^{n} \sum_{k=0}^{n} \frac{(-1)^{k}\left(\frac{1-\xi_{0}^{\prime}}{1+\xi_{0}^{\prime}}\right)^{k}}{\Gamma+1) \Gamma(1+n-k)} \cdot \\
\cdot \int_{0}^{\pi / 2} \frac{\cos 2\left(k+n_{2}\right) \psi P_{n+2 s}^{n}\left(\xi_{0} \cos \psi\right)}{\left(1-\xi_{0}^{2} \cos ^{2} \psi\right)_{2}^{n}} d \psi .
\end{aligned}
$$

Referring to the eq $94 \mathrm{a}$, we find that

Now

$$
\begin{aligned}
\frac{P_{n+2 s}^{n}\left(\xi_{0} \cos \psi\right)}{\left(1-\xi_{0}^{2} \cos ^{2} \psi\right)_{2}^{n}} & =\frac{(-1)^{s} 2^{n} \Gamma\left(s+n+\frac{1}{2}\right)}{\sqrt{\pi} \Gamma(s+1)} F\left(-s, s+n+\frac{1}{2}, \frac{1}{2} ; \xi_{0}^{2} \cos ^{2} \psi\right) \\
& =(-1)^{s} 2^{n} \sum_{t=0}^{s}(-1)^{t} \xi_{0}^{2 t} \frac{\Gamma\left(t+s+n+\frac{1}{2}\right) \cos ^{2 t} \psi}{\Gamma(1+s-t) \Gamma\left(t+1\left(\Gamma t t \frac{1}{2}\right)\right.} \cdot
\end{aligned}
$$

$$
\int_{0}^{\pi / 2} \frac{\cos 2\left(k+n_{2}\right) \psi \cos ^{2 t} \psi}{\Gamma(t+1) \Gamma\left(t+\frac{1}{2}\right)} d \psi=\frac{\sqrt{\pi}}{2 \Gamma\left(t+k+n_{2}+1\right) \Gamma\left(t-k-n_{2}+1\right)}
$$

Hence

$$
\begin{aligned}
& \int_{0}^{\pi / 2} \frac{\cos 2\left(k+n_{2}\right) \psi P_{n+2 s}^{n}\left(\xi_{0} \cos \psi\right)}{\left(1-\xi_{0}^{2} \cos ^{2} \psi\right)^{\frac{n}{2}}} d \psi \\
& =(-1)^{s} 2^{n-1} \sqrt{\pi} \sum_{t=0}^{8}(-1)^{t} \xi_{0}^{2 t} \frac{\Gamma\left(t+s+n_{2}+\frac{1}{2}\right)}{\Gamma(1+s-t) \Gamma\left(t+k+n_{2}+1\right) \Gamma\left(t-k-n_{2}+1\right)}
\end{aligned}
$$


Using this in eq 116 gives

$$
C_{s}^{n_{1}, n_{2}}=\frac{(-1)^{s}\left(1+\xi_{0}^{\prime}\right)^{n}}{2 \pi^{3 / 2} A_{1}^{2}} \sum_{t=0}^{s}(-1)^{t} \xi_{0}^{2 t} \frac{\Gamma\left(t+s+n+\frac{1}{2}\right)}{\Gamma(1+s-t)} S_{t},
$$

where

$$
S_{t} \equiv \Gamma(n+1) \sum_{k=0}^{n} \frac{(-1)^{k}\left(\frac{1-\xi_{0}^{\prime}}{1+\xi_{0}^{\prime}}\right)^{k}}{\Gamma(k+1) \Gamma\left(k+t+n_{2}+1\right) \Gamma\left(1+t-n_{2}-k\right) \Gamma(1+n-k)}
$$

In case $t \geqslant-n_{2}$, that is, $t+n_{2} \geqslant 0$,

$$
\begin{aligned}
S_{t}=\frac{F\left(-n, n_{2}-t, t+n_{2}+1 ;-\left(\frac{1-\xi_{0}^{\prime}}{1+\xi_{0}^{\prime}}\right)\right)}{\Gamma\left(t-n_{2}+1\right) \Gamma\left(t+n_{2}+1\right)} \\
=\left(\frac{1+\xi_{0}^{\prime}}{2}\right)^{n_{2}-t} \frac{F\left(n_{2}-t, t+n_{2}+n+1, t+n_{2}+1 ; \frac{1-\xi_{0}^{\prime}}{2}\right)}{\Gamma\left(t-n_{2}+1\right) \Gamma\left(t+n_{2}+1\right)}
\end{aligned}
$$

the last form being obtained by Euler's transformation of the first hypergeometric function. Since $t$ is never negative in eq 120 , it is evident that eq $122 \mathrm{a}$ is applicable whenever $n_{2} \geqslant 0$, and also for negative values of $n_{2}$ when the unequality $t>-n_{2}$ holds. In the remaining case, where $0 \leqslant t<-n_{2}$, we may write $n_{2}=-m$, where $m$ is a positive integer, so that, $0 \leqslant t \leqslant m$.

In this case

$$
\begin{aligned}
S_{t} & =\Gamma(n+1)(-1)^{m-t}\left(\frac{1-\xi_{0}^{\prime}}{1+\xi_{0}^{\prime}}\right)^{m-t} \frac{F\left(-2 t, m-t-n, m-t+1 ;-\left(\frac{1-\xi_{0}^{\prime}}{1+\xi_{0}^{\prime}}\right)\right)}{\Gamma(2 t+1) \Gamma(1+n-m+t) \Gamma(m-t+1)} \\
& =\Gamma(n+1)(-1)^{m}\left(\frac{1-\xi_{0}^{\prime}}{1+\xi_{0}^{\prime}}\right)^{m} \frac{(-1)^{t} \xi_{0}^{-2 t} 2^{2 t} F\left(-2 t, n+1, m-t+1 ; \frac{1-\xi_{0}^{\prime}}{2}\right)}{\Gamma(2 t+1) \Gamma(t+n-m+1) \Gamma(m-t+1)} .
\end{aligned}
$$

This expression however is that which eq122a becomes when $t+n_{2}<0$, so that either eq $122 \mathrm{a}$ or $122 \mathrm{~b}$ may be used, as they are both valid for positive and negative values of $n_{2}$. Using the latter, where $m$ is replaced by $-n_{2}$ gives

$(-1)^{t} \xi_{0}{ }^{2 t} S_{t}=$

$$
(-1)^{n_{2}}\left(\frac{1+\xi_{0}^{\prime}}{1-\xi_{0}^{\prime}}\right)^{n_{2}} \Gamma(n+1) \frac{2^{2 t} F\left(-2 t, n+1,1-n_{2}-t ; \frac{1-\xi_{0}^{\prime}}{2}\right)}{\Gamma\left(t+n+n_{2}+1\right) \Gamma(2 t+1) \Gamma\left(1-n_{2}-t\right)} .
$$


Using this, eq 120 becomes

$$
\begin{aligned}
& C_{s}^{n_{1}, n_{2}}= \frac{(-1)^{s+n_{2}}\left(1+\xi_{0}^{\prime}\right)^{n+n_{2}} \cdot\left(1-\xi_{0}^{\prime}\right)^{-n_{2}}}{2 \pi^{3 / 2} A_{1}^{2}} \Gamma(n+1) \\
&= \frac{\sum_{t=0}^{s} \frac{2^{2 t} \Gamma\left(t+s+n+\frac{1}{2}\right) F\left(-2 t, n+1,1-n_{2}-t ; \frac{1-\xi_{0}^{\prime}}{2}\right)}{\Gamma(1+s-t) \Gamma\left(t+n+n_{2}+1\right) \Gamma(2 t+1) \Gamma\left(1-n_{2}-t\right)}}{2 \pi^{s+2} A_{1}^{2}} \sum_{t=0}^{s+n_{2}\left(1+\xi_{0}^{\prime}\right) \frac{2^{2 t} \Gamma\left(t+s+n+\frac{1}{2}\right)}{\Gamma(1+s-t) \Gamma\left(t+n+n_{2}+1\right)}} \\
& \sum_{k=0}^{2 t} \frac{(-1)^{k}\left(\frac{1-\xi_{0}^{\prime}}{2}\right)^{k} \Gamma(k+n+1)}{\Gamma(k+1) \Gamma\left(k-t-n_{2}+1\right) \Gamma(1+2 t-k)} .
\end{aligned}
$$

Interchanging the order of summation gives

$$
C_{s}^{n_{1}, n_{2}}=\frac{(-1)^{s+n_{2}}\left(1+\xi_{0}^{\prime}\right)^{n+n_{2}}\left(1-\xi_{0}^{\prime}\right)^{-n_{2}}}{2 \pi^{3 / 2} A^{2}{ }_{1}^{2 s}} \sum_{k=0}(-1)^{k}\left(\frac{1-\xi_{0}^{\prime}}{2}\right)^{k} \frac{\Gamma(k+n+1)}{\Gamma(k+1)} G_{k},
$$

where

$$
G_{k} \equiv \sum_{t=0}^{s} \frac{2^{2 t} \Gamma\left(t+s+n+\frac{1}{2}\right)}{\Gamma(2 t+1-k) \Gamma\left(t+n+n_{2}+1\right) \Gamma(1+s-t) \Gamma\left(1+k-n_{2}-t\right)} \cdot
$$

In order to evaluate $G_{k}$ (eq 125) as a single term, we define the function $\mathrm{G}(z)$ as follows:

$$
\begin{aligned}
\mathscr{G}(z) & \equiv \sum_{k=-\infty}^{\infty}(-z)^{k} G_{k} \\
& \equiv \sum_{k=-\infty}^{\infty} \sum_{t=0}^{s} \frac{(-z)^{k} 2^{2 t} \Gamma\left(t+s+n+\frac{1}{2}\right)}{\Gamma(2 t+1-k) \Gamma\left(t+n+n_{2}+1\right) \Gamma(1+s-t) \Gamma\left(1+k-n_{2}-t\right)} .
\end{aligned}
$$

Since $n$ and $n_{2}$ are integers, this function is a polynomial in positive and negative powers of $z$. It is therefore analytic at every finite point in the $z$-plane except at $z=0$. After inverting the order of summation, we may substitute $k=\mu+n_{2}+t$ and sum first over $\mu$

$$
\begin{aligned}
\mathscr{G}(z)= & \sum_{t=0}^{s} \frac{(-z)^{n_{2}+t} 2^{2 t} \Gamma\left(t+s+n+\frac{1}{2}\right)}{\Gamma\left(t+n+n_{2}+1\right) \Gamma(1+s-t) \Gamma\left(t-n_{2}+1\right)} . \\
& \cdot \sum_{\mu=-\infty}^{\infty} \frac{(-z)^{\mu} \Gamma\left(t-n_{2}+1\right)}{\Gamma\left(t-n_{2}+1-\mu\right) \Gamma(\mu+1)}
\end{aligned}
$$

or

$$
\mathscr{G}(z)=\sum_{t=0}^{s} \frac{2^{2 t} \Gamma\left(t+s+n+\frac{1}{2}\right)(-z)^{n_{2}+t}(1-z)^{t-n_{2}}}{\Gamma\left(t+n+n_{2}+1\right) \Gamma(1+s-t) \Gamma\left(t-n_{2}+1\right)}
$$

This series starts not below $t=n_{2}$. Let $\lambda=t-n_{2}$, then

$$
\mathscr{G}(z)=(-2 z)^{2 n_{2}} \sum_{\lambda=0}^{\infty} \frac{[-4 z(1-z)]^{\lambda} \Gamma\left(\lambda+s+n+n_{2}+\frac{1}{2}\right)}{\Gamma(\lambda+1) \Gamma\left(\lambda+n+2 n_{2}+1\right) \Gamma\left(1+s-n_{2}-\lambda\right)} .
$$


The sum is of the form

$$
\sum_{i=0}^{\infty} \frac{[-4 z(1-z)]^{\lambda} \Gamma(\lambda+\alpha)}{\Gamma(\lambda+1) \Gamma\left(\lambda+\alpha+\beta+\frac{1}{2} \Gamma(1-\beta-\lambda)\right.}=\frac{\Gamma(\alpha) F\left(\alpha, \beta, \alpha+\beta+\frac{1}{2} ; 4 z(1-z)\right)}{\Gamma(1-\beta) \Gamma \alpha+\beta+\frac{1}{2}}
$$

with $\alpha=s+n+n_{2}+\frac{1}{2}$ and $\beta=n_{2}-s$.

By a special transformation due to Gauss

$$
F\left(\alpha, \beta, \alpha+\beta+\frac{1}{2} ; 4 z(1-z)\right)=F\left(2 \alpha, 2 \beta, \alpha+\beta+\frac{1}{2} ; z\right)
$$

and by use of the equation

$$
\begin{aligned}
& \frac{\Gamma(\alpha)}{\Gamma(1-\beta) \Gamma\left(\alpha+\beta+\frac{1}{2}\right)} F\left(2 \alpha, 2 \beta, \alpha+\beta+\frac{1}{2} ; z\right) \\
& \quad=\frac{2 \Gamma\left(\frac{1}{2}-\beta\right)}{2^{2 \alpha+2 \beta} \Gamma\left(\alpha+\frac{1}{2}\right)} \sum_{\lambda=0}^{\infty} \frac{(-z)^{\lambda} \Gamma(\lambda+2 \alpha)}{\Gamma(\lambda+1) \Gamma\left(\lambda+\alpha+\beta+\frac{1}{2}\right) \Gamma(1-2 \beta-\lambda)} .
\end{aligned}
$$

We get

$$
\begin{gathered}
\mathscr{G}(z)=\sum_{k=-\infty}^{\infty}(-z)^{k} G_{k} \\
=\frac{(-z)^{2 n_{2}} \Gamma\left(s-n_{2}+\frac{1}{2}\right)}{2^{2 n+2 n_{2}} \Gamma\left(s+n+n_{2}+1\right)} \sum_{\lambda=0}^{\infty} \frac{(-z)^{\lambda} \Gamma\left(\lambda+2 s+2 n+2 n_{2}+1\right)}{\Gamma(\lambda+1) \Gamma\left(\lambda+n+2 n_{2}+1\right) \Gamma\left(1+2 s-2 n_{2}-\lambda\right)}
\end{gathered}
$$

from which, by equating equal powers of $(z)$, we get for $G_{k}$ with $k=$ $\lambda+2 n_{2}$ the expression (eq 132) below. This brief method of deriving eq 132 from eq 125 was suggested to me by C. L. Critchfield, whose helpful criticism in other respects may be acknowledged here.

$\Gamma(k+n+1) G_{k}=\frac{\Gamma\left(s-n_{2}+\frac{1}{2}\right) \Gamma(k+2 s+2 n+1)}{2^{2 n+2 n_{2}} \Gamma\left(s+n+n_{2}+1\right) \Gamma(1+2 s-k) \Gamma\left(k+1-2 n_{2}\right)}$.

Using this in eq 124 gives

$$
\begin{aligned}
C_{s}{ }_{1}, n_{2}= & \frac{(-1)^{s+n_{2}} \Gamma\left(s-n_{2}+\frac{1}{2}\right)}{A_{1}^{2} \pi^{3 / 32} 2^{n+2 n_{2}+1} \Gamma\left(s+n+n_{2}+1\right)}\left(\frac{1+\xi_{0}^{\prime}}{2}\right)^{n} \cdot\left(\frac{1-\xi_{0}^{\prime}}{1+\xi_{0}^{\prime}}\right)^{-n_{2}} \cdot \\
& \cdot \sum_{k=0}^{2 s}(-1)^{k}\left(\frac{1-\xi_{0}^{\prime}}{2}\right)^{k} \frac{\Gamma(k+2 s+2 n+1)}{\Gamma(k+1) \Gamma\left(k+1-2 n_{2}\right) \Gamma(1+2 s+k)} .
\end{aligned}
$$

Now

$$
\begin{aligned}
& \sum_{k=0}^{2 s}(-1)^{k}\left(\frac{1-\xi_{0}^{\prime}}{2}\right)^{k} \frac{\Gamma(k+2 s+2 n+1)}{\Gamma(k+1) \Gamma\left(k+1-2 n_{2}\right) \Gamma(1+2 s-k)}= \\
& =\frac{\Gamma(2 s+2 n+1)}{\Gamma(2 s+1) \Gamma\left(1-2 n_{2}\right)} F\left(-2 s, 2 s+2 n+1,1-2 n_{2} ; \frac{1-\xi_{0}^{\prime}}{2}\right) \\
& =\left(\frac{a_{1}}{A_{1}}\right)^{2 s} \frac{\Gamma(2 s+2 n+1)}{\Gamma(2 s+1) \Gamma\left(1-2 n_{2}\right)} F\left(-2 s,-2 s-2 n-2 n_{2}, 1-2 n_{2} ;-\frac{a_{2}}{a_{1}}\right)
\end{aligned}
$$

by Euler's transformation, remembering that $1-\xi_{0}^{\prime}=2 a_{2} / A_{1}$ and $1+\xi_{0}^{\prime}=2 a_{1} / A_{1}$. 
We thus obtain the following

$$
\frac{2 \pi A_{1} \Gamma\left(s+\frac{1}{2}\right)}{2^{n} \Gamma(s+n+1)} \cdot C_{s}^{n_{1}, n_{2}}=\frac{(-1)^{n_{2}+s}}{A_{1} \pi^{3 / 2}} \cdot A_{2 s}^{2 n+2 n_{2},-2 n_{2}}\left(a_{1}, a_{2}\right),
$$

where the $A$-function is defined by

$$
\begin{aligned}
& A_{2 s}^{2 n_{1}, 2 n_{2}}\left(a_{1}, a_{2}\right) \equiv \\
& \equiv \frac{\Gamma\left(s+n_{1}+\frac{1}{2}\right) \Gamma\left(s+n_{2}+\frac{1}{2}\right) \Gamma\left(s+n_{1}+n_{2}+\frac{1}{2}\right) \Gamma\left(s+\frac{1}{2}\right)}{\Gamma(2 s+1) \Gamma\left(2 s+2 n_{1}+1\right) \Gamma\left(2 n_{2}+1\right)} \\
& \quad\left(\frac{a_{1}}{a_{1}+a_{2}}\right)^{n_{1}+2 s} \cdot\left(\frac{a_{2}}{a_{1}+a_{2}}\right)^{n_{2}} \cdot F\left(-2 s,-2 s-2 n_{1}, 2 n_{2}+1 ;-\frac{a_{2}}{a_{1}}\right)
\end{aligned}
$$

On expressing this as a polynomial and summing it in the reverse order, we find, since $s$ is an integer,

$$
\mathrm{A}_{2 s}^{2 n_{1}, 2 n_{2}}\left(a_{1}, a_{2}\right)=\mathrm{A}_{2 \boldsymbol{s}}^{2 n_{2}, 2 n_{1}}\left(a_{2}, a_{1}\right) .
$$

Also since $n_{2}$ is an integer, it is found that

$$
\mathrm{A}_{2 s}^{2 n_{1},-2 n_{2}}\left(a_{1}, a_{2}\right)=\mathrm{A}_{2\left(s-n_{2}\right)}^{2 n_{1}, 2 n_{2}}\left(a_{1}, a_{2}\right),
$$

which vanishes if $s-n_{2}<0$.

If $n_{2}=n_{1}$ in this, it is found that

$$
\mathrm{A}_{2 s}^{2 n_{1},-2 n_{1}}\left(a_{1}, a_{2}\right)=\mathrm{A}_{2 s}^{-2 n_{1}, 2 n_{1}}\left(a_{1}, a_{2}\right)=\frac{\pi \Gamma\left(s+\frac{1}{2}\right) \Gamma\left(s-n_{1}+\frac{1}{2}\right)}{2^{2 n_{1}} \Gamma(s+1) \Gamma\left(s+n_{1}+1\right)} \cdot \mathrm{P}_{2 s}^{2 n_{1}}\left(\xi_{0}^{\prime}\right),
$$

which vanishes when $s<n_{1}$.

Recalling that $n=n_{1}-n_{2} \geqslant 0$ and $n_{1} \geqslant 0$, it is evident that eq 134 may be written

$$
\frac{2 \pi A_{1} \Gamma\left(s+\frac{1}{2}\right)}{2^{n} \Gamma(s+n+1)} \mathrm{C}_{s}^{n_{1}, n_{2}}=\frac{(-1)^{n_{2}+s}}{A_{1} \pi^{\frac{3}{2}}} \mathrm{~A}_{2 s}^{2 n_{1},-2 n_{2}}\left(a_{1} a_{2}\right)
$$

In this equation $C_{s}^{n_{1}, n_{2}}$ is the value of $\int_{0}^{\xi_{0}} \xi_{1} P_{n+2 s}^{n}\left(\xi_{1}\right) \sigma\left(\xi_{1}\right) d \xi_{1}$, where the expression (eq 80) has been used for the density $\sigma$, so that with this value of the integral the formula (111) is the expansion of the function $U=\phi_{n_{1}, n_{2}}\left(x, r, a_{1}, a_{2}\right) / \sqrt{a_{1} a_{2}}$.

We have, therefore, obtained the following development when $n=n_{1}-n_{2} \geqslant 0$, which is valid for all positions of the two parallel circles, which converges to a finite value except in the case where the circles are equal in radii, and approach coincidence (in which case it becomes logarithmically infinite).

$$
\begin{aligned}
& \frac{\phi_{n_{1}, n_{2}}\left(x, r, a_{1}, a_{2}\right)}{\sqrt{a_{1}, a_{2}}} \\
& =\frac{(-1)^{n_{2}}}{\pi^{\frac{3}{2}} A_{1}} \sum_{s=0}^{\infty}(-1)^{s}\left(2 s+n+\frac{1}{2}\right) A_{2 s}^{2 n_{1},-2 n_{2}}\left(a_{1}, a_{2}\right) P_{n+2 s}^{n}(\xi) R_{n+2 s}^{n}(\eta) .
\end{aligned}
$$

When $n_{2}=n_{1} \geqslant 0$, this becomes by use of eq 138 
$\frac{\phi_{n_{1}, n_{1}}\left(x, r, a_{1}, a_{2}\right)}{\sqrt{a_{1} a_{2}}}=$

$\frac{(-1)^{n_{1}}}{2^{2 n_{1}} \sqrt{\pi} A_{1}} \sum_{s=n_{1}}^{\infty}\left(-1^{s}\right)\left(2 s+\frac{1}{2}\right) \frac{\Gamma\left(s+\frac{1}{2}\right) \Gamma\left(s-n_{1}+\frac{1}{2}\right)}{\Gamma(s+1) \Gamma\left(s+n_{1}+1\right)} P_{2 s}^{2 n_{1}}\left(\xi_{0}^{1}\right) P_{2 s}(\xi) R_{2 s}(\eta)$.

From eq 137 it is seen that the expansion (eq $\leqslant 140)$ begins with the term $s=n_{2}$ when $0 \leqslant n_{2} \leqslant n_{1}$, but with $s=0$ when $n_{1} \geqslant 0$ and $n_{2}<0$.

It may be noted that if $n \equiv\left|n_{1}-n_{2}\right|$ and if we write $A_{2 s}^{ \pm 2 n_{1}, \mp 2 n_{2}}\left(a_{1}, a_{2}\right)$ in eq 140, the expansion is valid for all integral values of $n_{1}$ and $n_{2}$, with the understanding that the upper of the double signs is to be taken when $n_{1}-n_{2}>0$, the lower when $n_{1}-n_{2}<0$. The ambiguity disappears when $n_{1}-n_{2}=0$.

From the integral representation (eq 57a) of $\frac{\phi n_{1} n_{2}}{\sqrt{a_{1} a_{2}}}$ it is evident that this function is unaltered if we interchange the arguments $a_{1}$ and $a_{2}$ and corresponding parameters $n_{1}$ and $n_{2}$ and multiply by $(-1)^{n_{1}-n_{2}}$. This fact is derivable from eq 140 by use of the relation eq 136 , noting that $A_{1}, A_{2}, \xi$, and $\eta$ are all unaffected by interchange of $a_{1}$ and $a_{2}$.

The integral representation shows also that

Where

$$
\frac{\phi_{n_{1}, n_{2}}\left(x, r, a_{1}, a_{2}\right)}{\sqrt{a_{1} a_{2}}}=\frac{(-1)^{n_{2}} \phi_{\bar{n}_{1}}, \bar{n}_{2}\left(x, a_{1}, r, a_{2}\right)}{\sqrt{r a_{2}}},
$$

$$
\left\{\begin{array}{l}
\bar{n}_{1}=n_{1}-n_{2} \\
\bar{n}_{2}=-\bar{n}_{2} \\
\bar{n}=\left|\bar{n}_{1}-\bar{n}_{2}\right|=\left|n_{1}\right|
\end{array}\right\}
$$

This interchange of arguments $r$ and $a_{1}$ in the light of eq 65 amounts to viewing $x$ and $a_{1}$ as the cylindrical coordinates of a point, while $r$ and $a_{2}$ are constants playing the part which $a_{1}$ and $a_{2}$ held in the preceding formulas.

Hence, as in eq 173 , we let

$$
\bar{A}_{1}=r+a_{2}, x=\bar{A}_{1} \vec{\xi} \vec{\eta}, a_{1}=\bar{A}_{1} \sqrt{\left(1-\bar{\xi}^{2}\right)\left(1+\bar{\eta}^{2}\right)},
$$

so that $\bar{\xi}$ and $\bar{\eta}$ are computed from the second members of eq 82 , after replacing $A_{1}$ by $r+a_{2}$ and $r$ by $a_{1}$.

The function on the right of eq 142 is then given by a development obtainable from eq 140 by replacing $A_{1}, a_{1}, \xi, \eta, n_{1}, n_{2}$, and $n$ by $\bar{A}_{1}$, $r, \bar{\xi}, \vec{\eta}, n_{1}-n_{2},-n_{2}$, and $\left|n_{1}\right|$, respectively. This gives

$$
\begin{aligned}
& \frac{\phi_{n_{1}, n_{2}}\left(x, r, a_{1}, a_{2}\right)}{\sqrt{a_{1} a_{2}}}=\frac{1}{\pi^{3 / 2}\left(r+a_{2}\right)} \\
& \left.\left.\sum_{s=0}^{\infty}(-1)^{s}\right) 2 s+\left|n_{1}\right|+\frac{1}{2}\right)\left.A_{2 s}^{ \pm 2\left(n_{1}-n_{2}\right), \pm 2 n_{2}}\left(r, a_{2}\right) P \cdot\left|n_{1}\right| \begin{array}{l}
n_{1} \mid+2 s \\
n_{1} \mid
\end{array}(\bar{\xi}) R\right|_{\left|n_{1}\right|+2 s} ^{\left|n_{1}\right|}(\bar{\eta}),
\end{aligned}
$$

the upper or lower sign being taken according as $n_{1}$ is positive or negative. Similarly, from interchange of $r$ and $a_{2}$,

$$
\frac{\phi_{n_{1}, n_{2}}\left(x, r, a_{1}, a_{2}\right)}{\sqrt{a_{1} a_{2}}}=\frac{\phi_{n_{1}} \bar{n}_{2}\left(x, a_{2}, a_{1}, r\right)}{\sqrt{a_{1} r}},
$$


where

$$
\left\{\begin{array}{l}
\bar{n}_{1}=n_{1} \\
\bar{n}_{2}=n_{1}-n_{2} \\
\bar{n}=\left|\bar{n}_{1}-\bar{n}_{2}\right|=\left|n_{2}\right|
\end{array}\right\}
$$

This gives a third expansion

$$
\begin{aligned}
& \frac{\phi_{n_{1}, n_{2}}\left(x, r, a_{1}, a_{2}\right)}{\sqrt{\overline{a_{1} a_{2}}}}=\frac{(-1)^{n_{1}-n_{2}}}{\pi^{3 / 2}\left(r+a_{1}\right)} \\
& \sum_{s=0}^{\infty}-\left.1^{s}\left(2 s+\left|n_{2}\right|+\frac{1}{2}\right) A_{2 s}\left(r, a_{1}\right)^{ \pm 2\left(n_{2}-n_{1}\right), \pm 2 n_{1}} \cdot P||_{n_{2} \mid+2 s}^{n_{2}}(\overline{\bar{\xi}}) \cdot R\right|_{n_{2} \mid+2 s} ^{\left|n_{2}\right|}\left(\begin{array}{l}
\bar{\eta} \\
)
\end{array}\right),
\end{aligned}
$$

where the upper or lower sign is taken according as $n_{2}$ is positive or negative

$$
x=\left(r+a_{1}\right) \overline{\overline{\xi \eta}} \text { and } a_{2}=\left(r+a_{1}\right) \cdot \sqrt{\left(1-\overline{\bar{\xi}}^{2}\right)\left(1+\overline{\bar{\eta}}^{2}\right)},
$$

so that $\overline{\bar{\xi}}$ and $\bar{\eta}$ are computed from the second members of eq 82 after replacing $A_{1}$ by $r+a_{1}$ and $r$ by $a_{2}$.

The following section is concerned with the partial integrals with respect to $x$ derivable from the expansion (eq 140).

\section{EXPANSION OF $\omega_{n_{1}, n_{2}}$ IN SPHEROIDAL COORDINATES}

The function $\omega_{n_{1}, n_{2}}\left(x, r, a_{1}, a_{2}\right)$, or more briefly, $\omega_{n_{1}, n_{2}}(x r)$ is represented in eq 59 by the integral

$$
\omega_{n_{1}, n_{2}}(x, r) \equiv 4 \pi^{2} \sqrt{a_{1} a_{2}} \int_{0}^{x} d x^{\prime \prime} \int_{0}^{x^{\prime \prime}} \phi_{n_{1}, n_{2}}\left(x^{\prime}, r\right) d x^{\prime} .
$$

We exclude for the present the exceptional case where $n_{1}$ and $n_{2}$ are both zero. In all other cases we may write

$$
\begin{gathered}
\omega_{n_{1}, n_{2}}(x, r)=V_{n_{1}, n_{2}}(x, r)-V_{n_{1}, n_{2}}(0, r)+2 \pi|x| S_{n_{1}, n_{2}}(r) \\
\omega_{n_{1}, n_{2}}^{\prime}(x, r)=V_{n_{1}, n_{2}}^{\prime}(x, r) \pm 2 \pi S_{n_{1}, n_{3}}(r),
\end{gathered}
$$

the upper or lower sign being taken according as $x$ is positive or negative. The function $V_{n_{1}, n_{2}}(x, r)$ is an even function of $x$ defined by

$$
V_{n_{1}, n_{2}}(x, r) \equiv 4 \pi^{2} a_{1} a_{2} \int_{0}^{\infty} e^{-|x| t} J_{n_{1}-n_{2}}(r t) J_{n_{1}}\left(a_{1} t\right) J_{n_{2}}\left(a_{2} t\right) \frac{d t}{t^{2}} .
$$

The function $V_{n_{1}, n_{2}}^{\prime}\left(x_{1} r\right)$ is an odd function of $x$ defined by

$$
V_{n_{1}, n_{3}}^{\prime}(x, r) \equiv \mp 4 \pi^{2} a_{1} a_{2} \int_{0}^{\infty} e^{-|x| t} J_{n_{1}-n_{2}}(r t) J_{n_{1}}\left(a_{1} t\right) J_{n_{2}}\left(a_{2} t\right) \frac{d t}{t}
$$


the upper or lower sign being taken according as $x$ is positive or negative. Also

$$
\begin{aligned}
2 \pi S_{n_{1}, n_{2}}(r)=-V_{n_{1}, n_{2}}^{\prime}(+0, r) & =+V_{n_{1}, n_{2}}^{\prime}(-0, r) \\
& =4 \pi^{2} a_{1} a_{2} \int_{0}^{\infty} J_{n_{1}-n_{2}}(r t) J_{n_{1}}\left(a_{1} t\right) J_{n_{2}}\left(a_{2} t\right) \frac{d t}{t} .
\end{aligned}
$$

These relations are all obtained by introducing into eq 148 the integral representation (eq 57a) for $\phi_{n_{1} n_{2}}$, which gives

$\omega_{n_{1}, n_{2}}(x, r)=4 \pi^{2} a_{1} a_{2} \int_{0}^{\infty}\left[\frac{e^{-|x| t}-1+|x| t}{t^{2}}\right] J_{n_{1}-n_{2}}(r t) J_{n_{1}}\left(a_{1} t\right) J_{n_{2}}\left(a_{2} t\right) d t$.

If the exceptional case $n_{1}=n_{2}=0$ be excluded, this may be separated into three integrals, each of which converges, thus giving the eq 149 to 153 .

From 152 and $57 \mathrm{a}$ it is evident that

if $x>0$,

$$
V_{n_{1}, n_{2}}^{\prime}(x, r)=-4 \pi^{2} \sqrt{a_{1} a_{2}} \int_{x}^{\infty} \phi_{n_{1}, n_{2}}\left(x^{\prime}, r\right) d x^{\prime}
$$

and

$$
V_{n_{1}, n_{3}}(x, r)=-\int_{x}^{\infty} V_{n_{1}, n_{2}}^{\prime}\left(x^{1}, r\right) d x^{\prime}
$$

if $x>0$. In both of these integrals, the integration must be performed, while $r$ is held constant.

The functions $V_{n_{1}, n_{2}}$ and $V_{n_{1}, n_{2}}^{\prime}$ each satisfy the partial differential eq 64. The function $V_{n_{1}, n_{2}}^{\prime}$ vanishes when the point $P(x, r)$ goes to infinity in any direction in the $(x, r)$ plane, while $V_{n_{1}, n_{2}}$ vanishes in general if $x \rightarrow \pm \infty$, while $r$ has any fixed finite value.

To perform the integrations in eq 155 we write eq 103 in the two forms:

$$
\begin{aligned}
& \left(2 s+n+\frac{1}{2}\right) P_{n+2 s}^{n}(\xi) R_{n+2 s}^{n}(\eta)= \\
& \quad-\frac{A_{1}}{2} D_{x}\left[P_{n+2 s-1}^{n}(\xi) \cdot R_{n+2 s-1}^{n}(\eta)+P_{n+2 s+1}^{n}(\xi) \cdot R_{n+2 s+1}^{n}(\eta)\right]
\end{aligned}
$$

$$
\begin{aligned}
\left(2 s+n+\frac{3}{2}\right) & P_{n+2 s+1}^{n}(\xi) R_{n+2 s+1}^{n}(\eta)= \\
& \quad-\frac{A_{1}}{2} D_{x}\left[P_{n+2 s}^{n}(\xi) \cdot R_{n+2 s}^{n}(\eta)+P_{n+2 s+2}^{n}(\xi) \cdot R_{n+2 s+2}^{n}(\eta)\right] .
\end{aligned}
$$

The expansion for $V_{n_{1}, n_{2}}^{\prime}$ is obtained by using the expansion (eq 140) for $\phi_{n_{1, n} n_{2}}$ in eq 155 and then performing the integration with respect to $x$ by use of eq 157. Since $r$ is held at a finite constant value in this integration, the value of the integrated terms at $x=\infty$ ( $r$ finite) are found by letting $\eta \rightarrow \infty$, while at the same time $\xi^{2} \rightarrow 1$, the two 
variables being connected, as shown in eq 81 , in such a manner that limit

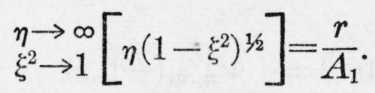

It is thus found that for the case $n \equiv n_{1}-n_{2}>0$

$$
\begin{aligned}
& V_{n_{1}, n_{2}}^{\prime}\left(x, r, a_{1}, a_{2}\right)= \\
& =(-1)^{n_{2}+1} 2 a_{1} a_{2} \sqrt{\pi}-\left\{\frac{A_{0}^{2 n_{1},-2 n_{2}}\left(a_{1}, a_{2}\right)}{n \sqrt{\pi}}\left(\frac{1-\xi^{2}}{1+\eta^{2}}\right)^{\frac{n}{2}} \xi F\left(1, n+\frac{1}{2}, n+1 ; 1-\xi^{2}\right)\right. \\
& \left.+\sum_{s=0}^{\infty}(-1)^{s}\left[A_{2 s}^{2 n_{1},-2 n_{2}}\left(a_{1}, a_{2}\right)-A_{2 s+2}^{2 n_{1},-2 n_{2}}\left(a_{1}, a_{2}\right)\right] P_{n+2 s+1}^{n}(\xi) R_{n+2 s+1}^{n}(\eta)\right\}
\end{aligned}
$$

and for the case $n_{2}=n_{1}>0(n=0)$

$$
\begin{array}{r}
\mathrm{V}_{n_{1}, n_{1}}^{\prime}\left(x, r, a_{1}, a_{2}\right)=(-1)^{n_{2}+1} 2 a_{1} a_{2} \sqrt{\pi} \sum_{s=n_{1}-1}^{\infty}(-1)^{s}\left[A_{2 s}{ }^{2 n_{1},-2 n_{1}}\left(a_{1}, a_{2}\right)\right. \\
\left.-A_{2 s+2}{ }^{2 n_{1},-2 n_{1}}\left(a_{1}, a_{2}\right)\right] P_{2 s+1}(\xi) R_{2 s+1}(\eta) .
\end{array}
$$

The first term inside the brace of eq 159 is

$$
\begin{aligned}
A_{0} 2 n_{1},-2 n_{2}\left(a_{1},\right. & \left.a_{2}\right)\left[P_{n+2 s-1}^{n}(\xi) R_{n+2 s-1}^{n}(\eta)\right]_{s=0} \\
= & \frac{A_{0}^{2 n_{1},-2 n_{2}}\left(a_{1}, a_{2}\right)}{n \sqrt{\pi}}\left(\frac{1-\xi^{2}}{1+\eta^{2}}\right)^{\frac{n}{2}} \xi F\left(1, n+\frac{1}{2}, n+1 ; 1-\xi^{2}\right),
\end{aligned}
$$

for from eq $95 \mathrm{~b}$ we find

$$
\left[\frac{P_{n+2 s-1}^{n}(\xi)}{s}\right]_{s=0}=\frac{2^{n}}{n \sqrt{\pi}} \Gamma\left(n+\frac{1}{2}\right)\left(1-\xi^{2}\right)^{\frac{n}{2}} \cdot \xi F\left(1, n+\frac{1}{2}, n+1 ; 1-\xi^{2}\right),
$$

and from the eq 99a we find

$$
\left[s R_{n+2 s-1}^{n}(\eta)\right]_{s=0}=\frac{\left(1+\eta^{2}\right)^{-\frac{n}{2}}}{2^{n} \Gamma\left(n+\frac{1}{2}\right)} .
$$

Also from eq 95a we find

$$
\left[\frac{1}{s} P_{n+2 s-2}^{n}(\xi)\right]_{3=0}=-\frac{2^{n-1} \Gamma\left(n-\frac{1}{2}\right)\left(1-\xi^{2}\right)^{\frac{n}{2}}}{n \sqrt{\pi}} \cdot F\left(1, n-\frac{1}{2}, n+1 ; 1-\xi^{2}\right),
$$

and from eq 99a

Hence

$$
\left[s R_{n+2 s-2}^{n}(\eta)\right]_{s=0}=-\frac{\eta}{2^{n-1} \Gamma\left(n-\frac{1}{2}\right)\left(1+\eta^{2}\right)^{\frac{n}{2}}}
$$

$$
\left[P_{n+2 s-2}^{n}(\xi) \cdot R_{n+2 s-2}^{n}(\eta)\right]_{s=0}=\frac{\eta}{n \sqrt{\pi}}\left(\frac{1-\xi^{2}}{1+\eta^{2}}\right)^{\frac{n}{2}} F\left(1, n-\frac{1}{2}, n+1 ; 1-\xi^{2}\right) .
$$


Inserting the expansion (eq 159) in (eq 156) and integrating by use of (eq 158) gives for the case $n>0$.

$$
\begin{aligned}
& V_{n_{1}, n_{2}}\left(x, r, a_{1}, a_{2}\right)= \\
& =(-1)^{n 2} a_{1} a_{2} A_{1} \sqrt{\pi}\left\{\frac{-A_{0}^{2 n_{1},-2 n_{2}}\left(a_{1}, a_{2}\right)}{n\left(n-\frac{1}{2}\right) \sqrt{\pi}}\left(\frac{1-\xi^{2}}{1+\eta^{2}}\right) \eta F\left(1, n-\frac{1}{2}, n+1 ; 1-\xi^{2}\right)\right. \\
& +\sum_{s=0}^{\infty}(-1)^{s}\left[\frac{A_{2 s}^{2 n_{1},-2 n_{2}}\left(a_{1}, a_{2}\right)-A_{2 s}^{2 n_{1}-2}-2 n_{2}\left(a_{1}, a_{2}\right)}{2 s+n+\frac{3}{2}}-\right. \\
& \left.\left.\frac{A_{2 s-2}^{2 n_{1},-2 n_{2}}\left(a_{1} a_{2}\right)-A_{2 s}^{2 n_{1},-2 n_{2}}\left(a_{1} a_{2}\right)}{2 s+n-\frac{1}{2}}\right] P_{n+2 s}^{n}(\xi) R_{n+2 s}^{n}(\eta)\right\}
\end{aligned}
$$

and for the case $n=0, n_{2}=n_{1}>0$

$$
\begin{aligned}
& V_{n_{1}, n_{1}}\left(x, r, a_{1}, a_{2}\right)= \\
& =(-1)^{n 1} a_{1} a_{2} A_{1} \sqrt{\pi} \sum_{s=n_{1}-1}^{\infty}(-1)^{s}\left[\frac{A_{2 s}^{2 n_{1},-2 n_{1}}\left(a_{1}, a_{2}\right)-A_{2 s+2}^{2 n_{1},-2 n_{2}}\left(a_{1}, a_{2}\right)}{2 s+\frac{3}{2}}-\right. \\
& \left.\qquad \frac{A_{2 s-2}^{2 n_{1},-2 n_{1}}\left(a_{1}, a_{2}\right)-A_{2 s}^{2 n_{1},-2 n_{1}}\left(a_{1}, a_{2}\right)}{2 s-\frac{1}{2}}\right] P_{2 s}(\xi) R_{2 s}(\eta) .
\end{aligned}
$$

The value of $V_{n_{1}, n_{2}}\left(0, r, a_{1}, a_{2}\right)$ for the range $0 \leqslant r \leqslant A_{1}$ is found by placing $\eta=0$ and $\xi=\sqrt{1-\frac{r^{2}}{A_{1}^{2}}}$ in eq 163 . For the range $r>A_{1}$ we place $\xi=0$ and $\eta=\sqrt{\frac{r_{1}^{2}}{A_{1}^{2}}-1}$. Similarly, from eq 159 the value of $2 \pi S_{n_{1} n_{2}}(r)=-V_{n_{1} n_{2}}^{\prime}\left(+0, r, a_{1} a_{2}\right)$ could be found, but such expansions are unnecessary, for we now find that $S_{n_{1} n_{2}}(r)$ may be obtained in finite form.

It should be noticed that the term $\xi F\left(1, n+1 / 2, n+1 ; 1-\xi^{2}\right)$ in eq 159 is an odd function of $\xi$ (and therefore of $x$ ), as are all the terms in $P_{n+28+1}^{n}(\xi)$ in the series. The latter all vanish with $\xi$, but the former does not, as may be seen by the transformation

$$
\xi F\left(1, n+\frac{1}{2}, n+1 ; 1-\xi^{2}\right)= \pm F\left(\frac{1}{2}, n, n+1 ; 1-\xi^{2}\right)=\frac{ \pm \sqrt{\pi} \Gamma(n+1)}{\Gamma\left(n+\frac{1}{2}\right)}
$$

when $\xi \rightarrow \pm 0$.

Hence when $x \rightarrow+0$ and $r>A_{1}$, i. e., $\xi \rightarrow+0$ and $\sqrt{\frac{1}{1+\eta^{2}}}=\frac{A_{1}}{r}$, eq 159 gives for $r>A_{1}$

$S_{n_{1}, n_{2}}(r)=-\frac{V_{n_{1} n_{2}}^{\prime}\left(+0, r, a_{1} a_{2}\right)}{2 \pi}=\frac{a_{1} a_{2}(-1)^{n_{2}} \Gamma(n)}{\sqrt{\pi} \Gamma\left(n+\frac{1}{2}\right)} A_{0}^{2 n_{1},-2 n_{2}}\left(a_{1}, a_{2}\right)\left(\frac{A_{1}}{r}\right)^{n}($

if $n \neq 0$. Referring to eq 135 , we find

$$
\frac{A_{0}^{2 n_{1},-2 n_{2}}\left(a_{1}, a_{2}\right)}{\sqrt{\pi} \Gamma\left(n+\frac{1}{2}\right)}=\frac{\pi}{A_{1}^{n}}\left(\frac{a_{1}^{n_{1}}}{\Gamma\left(1+n_{1}\right)}\right)\left(\frac{a_{2}^{-n_{2}}}{\Gamma\left(1-n_{2}\right)}\right)
$$

when $n_{1}>0$ and $n_{2}<n_{1}$. 
Hence eq 166 becomes

$$
S_{n_{1}, n_{\mathrm{g}}}(r)=\pi a_{1} a_{2}(-1)^{n_{2}} \frac{\Gamma(n)}{r^{n}}\left(\frac{a_{1}^{n_{1}}}{\Gamma\left(n_{1}+1\right)}\right)\left(\frac{a_{2}^{-n_{2}}}{\Gamma\left(1-n_{2}\right)}\right)
$$

when $r \geqslant A_{1}=a_{1}+a_{2}$ and $n_{1}>0, n_{2}<n_{1}$. This vanishes if $n_{2}$ is a positive integer less than $n_{1}$.

This relation may be used to obtain the value of $S_{n_{1} n_{2}}(r)$ for the range $0 \leqslant \mathrm{r}<A_{2}$ by considering the effect of interchanging $r$ with $a_{1}$ or $a_{2}$, which is determined by the integral definition (eq 153 of $S_{n_{1} n_{2}}(r)$, which is an abbreviation for $S_{n_{1} n_{2}}\left(r, a_{1}, a_{2}\right)$. It is thus found for the case $n_{1}>0$ and $n_{2}<n_{1}$ that when $0 \leqslant r \leqslant A_{2}=\left|a_{1}-a_{2}\right|$

$$
\left.\begin{array}{rlr}
S^{n_{1}, n_{2}}(r) & =\pi a_{1} a_{2} \frac{r^{n} \Gamma\left(n_{1}\right) a_{2}^{n_{2}}}{\Gamma(n+1) a_{1}^{n_{1}} \Gamma\left(n_{2}+1\right)}, & \text { when } a_{2}<a_{1} \\
& =0 & \text { when } a_{2}>a_{1}
\end{array}\right\}
$$

Similarly, for the case $n_{2}=n_{1}>0$ it is found that

$$
\left.\begin{array}{rl}
S_{n_{1}, n_{1}}(r) & =0 \text { when } A_{1} \leqslant r \leqslant \infty \\
& =\frac{\pi a_{1} a_{2}}{n_{1}}\left(\frac{a_{2}}{a_{1}}\right)^{\left|n_{1}\right|} \text { if } a_{2}<a_{1} \\
& =\frac{\pi a_{1} a_{2}}{n_{1}}\left(\frac{a_{1}}{a_{2}}\right)^{\left|n_{1}\right|} \text { if } a_{2}>a_{1}
\end{array}\right\} \text { when } 0 \leqslant r<A_{2} .
$$

In all these statements the ease $n_{1}=n_{2}=0$ is excluded. It is evident therefore that $S_{n_{1}, n_{2}}(r)$ vanishes when $r=\infty$ and also if $n_{1} \neq n_{2}$ when $r=0$. It may be shown from its definition (eq 153) that $S_{n_{1}, n_{2}}(r)$ is a continuous function of $r$, in particuler at $r=A_{1}$ and $r=A_{2}$. To find its value for the range $A_{2}<r<A_{1}$, we find from eq 152 that when $x>0$

$$
\begin{aligned}
& {\left[\frac{1}{r} D_{r}\left(r D_{r}\right)-\frac{n^{2}}{r^{2}}\right] \frac{V_{n_{1}, n_{2}}^{\prime}\left(x_{1} r\right)}{2 \pi}=-D_{x}^{2} \frac{V_{n_{1}, n_{2}}^{\prime}(x, r)}{2 \pi}} \\
& =2 \pi a_{1} a_{2} \int_{0}^{\infty} t e^{-x t} J_{n_{1}-n_{2}}(r t) J_{n_{1}}\left(a_{1} t\right) J_{n_{2}}\left(a_{2} t\right) d t .
\end{aligned}
$$

Letting $x$ approach zero in this, and noting that by eq 153 $V_{n_{1}, n_{2}}^{\prime}(+0, r)=-2 \pi S_{n_{1}, n_{2}}(r)$, and that the integral on the right approaches that which defines $2 \pi \sigma(r)$ in eq 66 , we find that $S_{n_{1}, n_{2}}(r)$ satisfies the ordinary nonhomogeneous differential equation

$$
\begin{gathered}
\frac{1}{r} D_{r}\left(r D_{r} S_{n_{1}, n_{2}}\right)-\frac{n^{2} S_{n_{1}, n_{2}}}{r^{2}}=-4 \pi^{2} a_{1} a_{2} \sigma(r) \\
=-2 \frac{\cos \left(n_{1} \theta_{1}^{0}-n_{2} \theta_{2}^{0}\right)}{\sin \left(\theta_{2}^{0}-\theta_{1}^{0}\right)}
\end{gathered}
$$

for the range $A_{2}<r<A_{1}$, this value of $\sigma$ being taken from eq 72 . If $r$ lies outside this range $\sigma_{n_{1} n_{2}}=0$, the homogeneous equation having solution $C_{1} r^{n}+C_{2} r^{-n}$ if $n \pm 0$ and $C_{1}+C_{2} \log r$ if $n=0$. The values just found are of this form. Hence if any particular integral of eq 171 is found for the range $A_{2}<r<A_{1}$, this increased by $C_{1} r^{n}+C_{2} r^{-n}$ is the 
general solution for that range, and the values of the two constants of integration, $C_{1}$ and $C_{2}$, are then determined by the fact that this solution must be continuous at $r=A_{2}$ and at $r=A_{1}$ with the solutions previously found and given by eq 168 and 169. Similarly, in the case $n=0$.

To derive a particular integral of eq 171 in finite form for the case $n_{1}>0$ and $n_{2}<n_{1}$, so that $n=n_{1}-n_{2}>0$, we begin with the elementary integral form of the solution

$$
S(r)=\frac{a_{1} a_{2}}{n}\left[r^{-n} I_{1}-r^{n} I_{2}\right],
$$

where

$$
\begin{aligned}
& I_{1}=\int r^{n} \cos \left(n_{1} \theta_{1}^{0}-n_{2} \theta_{2}^{0}\right) d 2 \psi \\
& I_{2}=\int r^{-n} \cos \left(n_{1} \theta_{1}^{0}-n_{2} \theta_{2}^{0}\right) d 2 \psi
\end{aligned}
$$

Now by eq 77

$$
\begin{gathered}
r^{n} \cos \left(n_{1} \theta_{1}^{0}-n_{2} \theta_{2}^{0}\right)=\mathscr{R} a_{1}^{n} e^{-2 i n_{2} \psi}\left(1-\frac{a_{2} e^{-2 i \psi}}{a_{1}}\right)^{n} \\
=a_{1}^{n} \Gamma(n+1) \sum_{k=0}^{n} \frac{(-1)^{k}\left(\frac{a_{2}}{a_{1}}\right)^{k} \cos 2\left(k+n_{2}\right) \psi}{\Gamma(k+1) \Gamma(1+n-k)} \\
=(-1) n_{2}^{n_{2}} \frac{a_{1}^{n_{1}}}{a_{2}^{n_{2}}} \Gamma(n+1) \sum_{k=n_{2}}^{n_{1}} \frac{(-1)^{k}\left(\frac{a_{2}}{a_{1}}\right)^{k} \cos 2 k \psi}{\left.\Gamma-n_{2}+1\right) \Gamma\left(1+n_{1}-k\right)},
\end{gathered}
$$

so that

$$
\begin{gathered}
r^{-n} I_{1}=(-1)^{n_{2}} \frac{\Gamma(n+1)}{r^{n}} a_{1}{ }^{n_{1}} a_{2}{ }^{-n_{2}}\left\{\frac{2 \psi}{\Gamma\left(n_{1}+1\right) \Gamma\left(1-n_{2}\right)}+\right. \\
\left.\sum_{k=n_{2}}^{n_{1}} \frac{(-1)^{k}\left(\frac{a_{2}}{a_{1}}\right)^{k}}{\Gamma\left(k-n_{2}+1\right) \Gamma\left(1+n_{1}-k\right)} \frac{\sin 2 k \psi}{k}\right\},
\end{gathered}
$$

where $\Sigma^{\prime}$ indicates that the term $k=0$ is omitted. To evaluate $I_{2}$ we derive from eq 77

and

$$
r^{-n} \cos \left(n_{1} \theta_{1}^{0}-n_{2} \theta_{2}^{0}\right)=\mathscr{R} \frac{a_{2}^{n_{2}}}{a_{1}^{n_{1}}}\left(\frac{r e^{i \theta_{1}^{0}}}{a_{2}}\right)^{-n} \cdot\left(1+\frac{r e^{i \theta_{2}^{0}}}{a_{2}}\right)^{n 1}
$$

so that

$$
d 2 \psi=-i\left(1+\frac{r e^{i \theta_{2}^{0}}}{a_{2}}\right)^{-1} d\left(\frac{r e^{i \theta_{2}^{0}}}{a_{2}}\right)
$$

$$
I_{2}=-\frac{i a_{2}{ }_{2}}{a_{1}{ }^{n_{1}}} \int\left(\frac{r e^{i \theta_{2}^{0}}}{a_{2}}\right)\left[1+\frac{r e^{i \theta_{2}^{0}}}{a_{2}}\right]^{n_{1}^{-1}} d\left(\frac{r e^{i \theta_{2}^{0}}}{a_{2}}\right)
$$

where the real part is to be taken.

$$
\text { Since } n_{1}>0 \text {, the expansion of the binomial }\left[1+\frac{r e^{i \theta_{2}^{0}}}{a_{2}}\right]^{n_{1}-1} \text { is a finite }
$$


polynomial in $r e^{i \theta_{2}^{0}} a_{2}$. This gives

$$
\begin{aligned}
& r^{n} I_{2}=\frac{r^{n} \Gamma\left(n_{1}\right) a_{2}{ }^{n_{2}}}{a_{1}{ }^{n_{1}}}\left\{\frac{\theta_{2}^{0}}{\Gamma(n) \Gamma\left(n_{2}+1\right)}+\right. \\
& \left.\sum_{k=-n_{2}}^{n-1} \frac{\left(\frac{a_{2}}{r}\right)^{k}}{\Gamma\left(k+n_{2}+1\right) \Gamma(n-k)} \frac{\sin k \theta_{2}^{0}}{k}\right\},
\end{aligned}
$$

Using eq 173 and 174 in eq 172 gives a particular integral $S(r)$ of eq 171 for the range $A_{2}<r<A_{1}$, when $n_{1}>0$ and $n_{2}<n_{1}$. Hence if $A_{2} \leqslant r<A_{1}, n_{1}>0$ and $n_{2}<n_{1}$, we find

$S_{n_{1} n_{2}}(r)=$

$=a_{1} a_{2}\left\{\frac{(-1){ }_{2}{ }_{2} \Gamma(n) a_{1}^{n_{1}} a_{2}^{-n}}{\mathrm{r}^{n}}\left[\frac{2 \psi}{\Gamma\left(n_{1}+1\right) \Gamma\left(1-n_{2}\right)}\right.\right.$

$$
\begin{aligned}
& \left.+\sum_{k=n_{2}}^{n_{1}} \frac{(-1)^{k}\left(\frac{a_{2}}{a_{1}}\right)^{k}}{\Gamma\left(k-n_{2}+1\right) \Gamma\left(1+n_{1}-k\right)} \frac{\sin 2 k \psi}{k}\right] \\
& +\frac{r^{n} \Gamma\left(n_{1}\right) a_{2}^{n_{2}}}{a_{1}^{n_{1}}}\left[\frac{\pi-\Theta_{2}^{0}}{\Gamma(n+1) \Gamma\left(n_{2}+1\right)}\right. \\
& \left.\left.-\frac{1}{n} \sum_{k=-n_{2}}^{n-1} \frac{\left(\frac{a_{2}}{r}\right)^{k}}{\Gamma\left(k+n_{2}+1\right) \Gamma(n-k)} \frac{\sin k \theta_{2}^{0}}{k}\right]\right\} \text {, }
\end{aligned}
$$

where the constants $C_{1}$ and $C_{2}$ have been so chosen as to make $S_{n_{1} n_{3}}(r)$ continuous at $r=A_{1}$ and at $r=A_{2}$, its values at these points being given by eq 168 and 169 when $r=A_{1}$ or $A_{2}$, respectively.

The terms in the summation vanish at each of these values of $r$, for, when $r=A_{1}, \theta_{1}^{0}=0, \theta_{2}^{0}=\pi$, and $2 \psi=\theta_{2}^{0}-\theta_{1}^{0}=\pi$, and when $\mathrm{r}=A_{2}$,

$2 \psi=0$, and $\left\{\begin{array}{c}\theta_{1}^{0}=\theta_{2}^{0}=0 \text { if } a_{2}<a_{1} \\ \theta_{1}^{0}=\theta_{2}^{0}=\pi \text { if } a_{2}>a_{1}\end{array}\right\}$.

This shows that the second member of eq 175 reduces to eq 168 when $r=A_{1}$ and to eq 169 when $r=A_{2}$. Therefore, eq 168, 169, and 175 give $S r_{n 1 n 2}$ in finite terms for all values of $r$ when $n_{1}>0$ and $n_{2}<n_{1}$.

In eq $175 S_{n_{1} n_{2}}(r)$ is $S_{n_{1} n_{2}}\left(r, a_{1} a_{2}\right)$. In order to find $S_{n_{1} n_{2}}\left(r, a_{2}, a_{1}\right)$, we must not only interchange $a_{1}$ and $a_{2}$ where they occur explicitly in the second member of eq 172 but must also replace $\theta_{2}$ by $\pi-\theta_{1}$, as shown by eq $78 \mathrm{~b}$. 
The remaining irreducible cases are for $n_{2}=n_{1}>0$ and $n_{2}=n_{1}=0$. In the case $n_{2}=n_{1}>0(n=0)$, we start with the particular integral of eq 171

$S(r)=4 n^{2} a_{1} a_{2} \int r_{1} \sigma\left(r_{1}\right) \log \frac{r_{1}}{r} d r_{1}=2 a_{1} a_{2} \int \cos 2 n_{1} \psi^{\prime} \log \frac{r_{1}}{r} d 2 \psi^{\prime}$

$=a_{1} a_{2}\left\{\frac{2 \sin 2 n_{1} \psi}{n_{1}} \log \frac{1}{r}+2 \int \cos 2 n_{1} \psi^{\prime} \log r_{1} d 2 \psi^{\prime}\right\}$

$=a_{1} a_{2}\left\{\frac{2 \sin 2 n_{1} \psi}{n_{1}} \log \frac{a_{1}}{r}-2 \sum_{k=1}^{\infty} \frac{1}{k}\left(\frac{a_{2}}{a_{1}}\right)^{k} \int \cos 2 n_{1} \psi^{\prime} \cos 2 k \psi^{\prime} d 2 \psi^{\prime}\right\}$

$=a_{1} a_{2}\left\{\frac{2 \sin 2 n_{1} \psi}{n_{1}} \log \frac{a_{1}}{r}\right.$

$$
\left.-\sum_{k=1}^{\infty} \frac{1}{k}\left(\frac{a_{2}}{a_{1}}\right)^{k}\left[\frac{\sin 2\left(k+n_{1}\right) \psi}{k+n_{1}}+\frac{\sin 2\left(k-n_{1}\right) \psi}{k-n_{1}}\right]\right\} .
$$

This may be reduced to finite terms by use of the expansion

$\frac{2 \sin 2 n_{1} \psi}{n_{1}} \log \frac{a_{1}}{r}=\sum_{k=1}^{\infty} \frac{1}{k}\left(\frac{a_{2}}{a_{1}}\right)^{k} \frac{2 \sin 2 n_{1} \psi \cos 2 k \psi}{n_{1}}$

$$
=\sum_{k=1}^{\infty} \frac{1}{k}\left(\frac{a_{2}}{a_{1}}\right)^{k}\left[\frac{\sin 2\left(k+n_{1}\right) \psi}{n_{1}}-\frac{\sin 2\left(k-n_{1}\right) \psi}{n_{1}}\right] \text {, }
$$

so that

$$
\begin{aligned}
& S(r)= \frac{a_{1} a_{2}}{n_{1}} \sum_{k=1}^{\infty}\left(\frac{a_{2}}{a_{1}}\right)^{k}\left[\frac{\sin 2\left(k+n_{1}\right) \psi}{k+n_{1}}-\frac{\sin 2\left(k-n_{1}\right) \psi}{k-n_{1}}\right] \\
&=\frac{a_{1} a_{2}}{n_{1}}\left\{-2 \psi\left(\frac{a_{2}}{a_{1}}\right)^{n_{1}}-\left[\left(\frac{a_{2}}{a_{1}}\right)^{n_{1}}-\left(\frac{a_{1}}{a_{2}}\right)^{n_{1}}\right] \sum_{k=1}^{\infty}\left(\frac{a_{2}}{a_{1}}\right)^{k} \frac{\sin 2 k \psi}{k}\right. \\
&\left.-\sum_{k=1}^{n_{1}}\left[\left(\frac{a_{2}}{a_{1}}\right)^{k-n_{1}}+\left(\frac{a_{1}}{a_{2}}\right)^{k-n_{1}}\right] \frac{\sin 2 k \psi}{k}+\frac{\sin 2 n_{1} \psi}{n_{1}}\right\}
\end{aligned}
$$

Now

$$
e^{i \theta_{1}^{0}}=\left(\frac{1-\frac{a_{2}}{a_{1}} e^{-2 i \psi}}{1-\frac{a_{2}}{a_{1}} e^{2 i \psi}}\right)^{\frac{1}{\sigma}},
$$

so that taking the logarithm of both sides we obtain

$$
\theta_{1}^{0}=\sum_{k=1}^{\infty}\left(\frac{a_{2}}{a_{1}}\right)^{k} \frac{\sin 2 k \psi}{k}
$$

The particular integral then becomes for the case $n_{2}=n_{1}>0$.

$S(r)=\frac{a_{1} a_{2}}{n_{1}}$

$$
\left\{\left(\frac{a_{1}}{a_{2}}\right)^{n_{1}} \theta_{1}^{0}-\left(\frac{a_{2}}{a_{1}}\right)^{n_{1}} \theta_{2}^{0}+\frac{\sin 2 n_{1} \psi}{n_{1}}-\sum_{k=1}^{n_{1}}\left[\left(\frac{a_{2}}{a_{1}}\right)^{k-n_{1}}+\left(\frac{a_{1}}{a_{2}}\right)^{k-n_{1}}\right] \frac{\sin 2 k \psi}{k}\right\},
$$


which contains only a finite number of terms and is valid whichever of the two circles is the larger.

With this particular integral we find, after determining the two constants of integration by making $S_{n_{1} n_{2}}(r)$ continuous at $r=A_{2}$ and at $r=A_{1}$ with the values given in eq 170 ,

$$
\begin{aligned}
S_{n_{1} n_{2}}(r) & =\frac{\pi a_{1} a_{2}}{n_{1}}\left(\frac{a_{2}}{a_{1}}\right)^{n_{1}} \text { if } a_{2}<a_{1} \mid \text { when } 0 \leqslant r \leqslant A_{2}, \\
= & \left.\frac{\pi a_{1} a_{2}}{n_{1}}\left(\frac{a_{1}}{a_{2}}\right)^{n_{1}} \text { if } a_{1}<a_{2}\right\} \\
= & \frac{a_{1} a_{2}}{n_{1}}\left\{\left(\frac{a_{1}}{a_{2}}\right)^{n_{1}} \theta_{1}^{0}+\left(\frac{a_{2}}{a_{1}}\right)^{n_{1}}\left(\pi-\theta_{2}^{0}\right)+\frac{\sin 2 n_{1} \psi}{n_{1}}\right. \\
-\sum_{k=1}^{n_{1}}\left[\left(\frac{a_{2}}{a_{1}}\right)^{k-n_{1}}+\left(\frac{a_{1}}{a_{2}}\right)^{k-n_{1}}\right] & \left.\frac{\sin 2 k \psi}{k}\right\} \\
=0 & \text { when } A_{2} \leqslant r \leqslant A_{1}
\end{aligned}
$$

provided that $n_{1}>0$.

In the particular case of greatest importance, $n_{1}=1$,

$$
\begin{array}{rlr}
S_{11}(r) & =\left\{\frac{\pi a_{2}^{2} \text { if } a_{2}<a_{1}}{\pi a_{1}^{2} \text { if } a_{1}<a_{2}}\right\} & \text { when } 0 \leqslant r \leqslant A_{2} \\
& =a_{1}^{2} \theta_{1}^{0}+a_{2}^{2}\left(\pi-\theta_{2}^{0}\right)-a_{1} a_{2} \sin 2 \psi & \text { when } A_{2} \leqslant r \leqslant A_{1} \\
& =0 & \text { when } A_{1} \leqslant r \leqslant \infty .
\end{array}
$$

This shows that $S_{11}(r)$ is the area common to the two coplanar circles of radii $a_{1}$ and $a_{2}$, when $r$ is the distance between their axes.

By the preceding formulas every $\omega_{n_{1} n_{2}}$ is evaluated with the exception of the case $n_{1}=n_{2}=0$. Before considering it, attention may be called to the interesting fact that although every $V_{n_{1}, n_{2}}$ vanishes when $x \rightarrow \pm \infty$ holding $r$ finite, there are two cases of eq 163 namely, $V_{1,0}\left(x, r, a_{1}, a_{2}\right)$ and $V_{0,-1}\left(x, r, a_{1}, a_{2}\right)$ which do not vanish when the point $P(x, r)$ goes to infinity in directions other than the $x$-direction.

By use of eq 163 and 167 it is found that when $x^{2}+r^{2} \rightarrow \infty$ in the direction $\phi$ defined by $\cos \phi=\frac{x}{\sqrt{x^{2}+r^{2}}}$ then

$$
\left.\begin{array}{r}
V_{1,0}\left(x, r, a_{1}, a_{2}\right) \rightarrow 2 \pi^{2} a_{1}^{2} a_{2}\left|\tan \frac{\phi}{2}\right| \\
V_{0,-1}\left(x, r, a_{1}, a_{2}\right) \rightarrow-2 \pi^{2} a_{1} a_{2}^{2}\left|\tan \frac{\phi}{2}\right|
\end{array}\right\} .
$$

In the exceptional case where $n_{2}=n_{1}=0$, the integrals involving Bessel's functions, which served to define the functions $V_{n_{1}, n_{2}}$ and $V_{n_{1}, n_{\mathrm{s}}}^{\prime}$ in eq 151 and 152 , are not convergent. Neither are the definitions of these functions by the integrals, eq 155 and 156 . Nevertheless, functions

$$
V_{00}(x, r), V_{00}^{\prime}(x, r) \equiv D_{x} V_{00}(x, r), \text { and } S_{00}(r)
$$


may be defined in this case, so that eq 149 and 150 are applicable. To find these functions we begin with

$$
\begin{gathered}
\omega_{00}(x, r)=\int_{0}^{x} \omega_{00}^{\prime}\left(x^{\prime}, r\right) d x^{\prime}=4 \pi^{2} a_{1} a_{2} \int_{0}^{x} d x^{\prime \prime} \int_{0}^{x^{\prime \prime}} \frac{\phi_{00}\left(x^{\prime}, r\right)}{\sqrt{a_{1} a_{2}}} d x^{\prime} \\
\omega_{00}^{\prime}(x, r)=D_{x} \omega_{00}(x, r)=4 \pi^{2} a_{1} a_{2} \int_{0}^{x} \frac{\phi_{00}\left(x^{\prime}, r\right)}{\sqrt{a_{1} a_{2}}} d x^{\prime} .
\end{gathered}
$$

Applying the operator $r^{-1} D_{\tau}\left(r D_{\tau}\right)$ to the latter gives

$$
\begin{aligned}
\frac{1}{r} D_{r}\left(r D_{r} \omega_{00}^{\prime}\right) & =4 \pi^{2} a_{1} a_{2} \int_{0}^{x} \frac{1}{r} D_{r}\left(\frac{r D_{r} \phi_{00}\left(x^{\prime}, r\right)}{\sqrt{a_{1} a_{2}}}\right) d x^{\prime} \\
& =-4 \pi^{2} a_{1} a_{2} \int_{0}^{x} D_{x^{\prime}}^{2}\left(\frac{\phi_{00}\left(x^{\prime}, r\right)}{\sqrt{a_{1} a_{2}}}\right) d x^{\prime} \\
& =-4 \pi^{2} a_{1} a_{2} D_{x}\left(\frac{\phi_{00}(x, r)}{\sqrt{a_{1} a_{2}}}\right)+4 \pi^{2} a_{1} a_{2}\left[D_{x}\left(\frac{\phi_{00}(x, r)}{\sqrt{a_{1} a_{2}}}\right)\right]_{x \rightarrow 0} .
\end{aligned}
$$

Also applying the operator $D_{x}^{2}$ to eq 182 gives

so that

$$
D_{x}^{2} \omega_{00}^{\prime}=4 \pi^{2} a_{1} a_{2} D_{x} \frac{\phi_{00}(x, r)}{\sqrt{a_{1} a_{2}}},
$$

$$
\nabla^{2} \omega_{00}^{\prime}(x, r)=\left[D_{x}^{2}+\frac{1}{r} D_{r}\left(r D_{r}\right)\right] \omega_{00}^{\prime}(x, r)=4 \pi^{2} a_{1} a_{2}\left[D_{x} \frac{\phi_{00}(x, r)}{\sqrt{a_{1} a_{2}}}\right]_{x \rightarrow 0} .
$$

That is, by eq 66

$$
\nabla^{2} \omega_{00}^{\prime}(x, r)=\mp 4 \pi^{2} a_{1} a_{2} .2 \pi \sigma_{00}(r)
$$

the upper sign if $x>0$ the lower if $x<0$. This shows that $\omega_{00}^{\prime}(x, r)$ is of the form as in eq 150

$$
\omega_{00}^{\prime}(x, r)=V_{00}^{\prime}(x, r) \pm 2 \pi S_{00}(r),
$$

where $V_{00}^{\prime}(x, r)$ is an odd function of $x$ satisfying

$$
\nabla^{2} V_{00}^{\prime}(x, r)=0,
$$

and $S_{00}(r)$ is a solution of

$$
\nabla^{2} S_{00}(r)=\frac{1}{r} D_{r}\left(r D_{r} S_{00}(r)\right)=-4 \pi_{a 1}^{2} a_{2} \sigma_{00}(r)=\frac{-2}{\sin 2 \psi},
$$

as in eq 171.

Since $\omega_{00}^{\prime}$ and $\omega_{00}$ both vanish with $x$,

as in eq 153.

$$
S_{00}(r)=-\frac{V_{00}^{\prime}(+0, r)}{2 \pi}=+\frac{V_{00}^{\prime}(-0, r)}{2 \pi},
$$

Also by eq 184

$$
\omega_{00}(x, r)=\int_{0} \omega_{00}^{\prime}\left(x^{\prime}, r\right) d x^{\prime}=V_{00}(x, r)-V_{00}(0, r)+2 \pi|x| S_{00}(r),
$$

as in eq 149 . 
If in the integral (eq 182) we introduce for $\phi_{00}\left(x^{\prime}, r\right) / \sqrt{a_{1} a_{2}}$ its expansion (eq 140), where $A_{2 s}^{0.0}=\frac{\pi \Gamma^{2}\left(s+\frac{1}{2}\right)}{\Gamma^{2}(s+1)} P_{2 s}\left(\xi_{0}^{\prime}\right)$ by eq 138 , this gives

$$
\begin{aligned}
& \omega_{00}^{\prime}(x, r)=\frac{4 a_{1} a_{2} \sqrt{\pi}}{A_{1}} \\
& \left\{\frac{\pi^{2}}{2} \int_{0}^{x} P_{0}(\xi) R_{0}(\eta) d x+\sum_{s=1}^{\infty}(-1)^{s}\left(2 s+\frac{1}{2}\right) A_{2 s}^{0,0}\left(a_{1}, a_{2}\right) \int_{0}^{x} P_{2 s}(\xi) R_{2 s}(\eta) d x\right\}
\end{aligned}
$$

where

$$
P_{0}(\xi) R_{0}(\eta)=R_{0}(\eta)=\frac{2}{\sqrt{\pi}} \cot ^{-1} \eta
$$

The terms in the series of eq 189 , for which $s>0$, may be integrated by the use of formula 157, but the present case is again exceptional for eq 157 now fails entirely, for the term $s=0$ becomes infinite instead of merely becoming indeterminate of the form $0 / 0$, as in the case of $P_{s}^{n}$, where $n=0$.

This breakdown of the formula may be repaired by writing

$$
P_{0}(\xi) R_{0}(\eta)=\frac{1}{2 \pi i} \int_{\mu=0} P_{\mu}(\xi) R_{\mu}(\eta) \frac{d \mu}{\mu},
$$

where the integral is a contour integral taken around a small closed curve in the complex $\mu$-plane, encircling the point $\mu=0$. Since $\mu$ does not vanish at any point on this curve, the integrand may be written by eq 103

$$
P_{\mu}(\xi) R_{\mu}(\eta)=-\frac{A_{1}}{2 \mu+1} D_{x}\left[P_{\mu-1}(\xi) R_{\mu-1}(\eta)+P_{\mu+1}(\xi) R_{\mu+1}(\eta)\right],
$$

which gives by Cauchy's theorem

$$
P_{0}(\xi) R_{0}(\eta)=\frac{2}{\sqrt{\pi}} \cot ^{-1} \eta=-A_{1} D_{x}\left\{D_{\mu}\left[\frac{\mu P_{\mu-1}(\xi) R_{\mu-1}(\eta)}{2 \mu+1}\right]_{\mu-0}+P_{1}(\xi) R_{1}(\eta)\right\} \cdot
$$

It is found that

$$
\frac{\sqrt{\pi}}{2} D_{\mu}\left[\frac{\mu P_{\mu-1}(\xi) R_{\mu-1}(\eta)}{2 \mu+1}\right]=-\log \frac{(1+\xi) \sqrt{1+\eta^{2}}}{2} .
$$

If we define the function $v(x, r)$ by

$$
\left.\begin{array}{rl}
v(x, r) & \equiv-\frac{2}{\sqrt{\pi}} \log \left[(1+\xi) \cdot \sqrt{1+\eta^{2}}\right] \text { when } x>0 \\
& \equiv+\frac{2}{\sqrt{\pi}} \log \left[(1-\xi) \sqrt{1+\eta^{2}}\right] \text { when } x<0
\end{array}\right\}
$$

then $v(x, r)$ is an odd function of $x$, which is an axially symmetrical solution of Laplace's equation, and eq 192 becomes

$$
P_{0}(\xi) R_{0}(\eta)=\frac{2}{\sqrt{\pi}} \cot ^{1} \eta=-A_{1} D_{x}\left\{v(x, r)+P_{1}(\xi) R_{1}(\eta)\right\} .
$$

A further integration may be made by the same method. This gives

$$
v(x, r)=D_{x}\left\{x v(x, r)+\frac{2 A_{1}}{\sqrt{\pi}}\left(\eta+\cot ^{-1} \eta\right)\right\} .
$$


The function within the parentheses of eq 196 is an even function of $x$, satisfying Laplace's equation. Integrating eq 189 by use of eq 157 and 195 leads to the following definition of $V_{00}^{\prime}$

$$
\begin{aligned}
& V_{00}^{\prime}(x, r)=-2 a_{1} a_{2} \sqrt{\pi} \\
& \quad\left\{\pi^{2} v(x, r)+\sum_{s=0}^{\infty}(-1)^{s}\left[A_{2 s}^{00}\left(a_{1} a_{2}\right)-A_{2 s+2}^{00}\left(a_{1} a_{2}\right)\right] P_{2 s+1}(\xi) R_{2 s+1}(\eta)\right\} .
\end{aligned}
$$

Integrating this from $x=0$ to $x=x$ by eq 158 and 196 leads to

$$
\begin{aligned}
& V_{00}(x, r)=-a_{1} a_{2} \sqrt{\pi}\left\{2 \pi^{2}\left[x v(x, r)+\frac{2 A_{1}}{\sqrt{\pi}} \eta\right]+A_{1} \sum_{s=0}^{\infty}(-1)^{s}\right. \\
& \left.\left[\frac{A_{2 s-2}^{00}\left(a_{1}, a_{2}\right)-A_{2 s}^{00}\left(a_{1}, a_{2}\right)}{2 s-\frac{1}{2}}-\frac{A_{2 s}^{00}\left(a_{1}, a_{2}\right)-A_{2 s}^{r, 0}\left(a_{1}, a_{2}\right)}{2 s+\frac{s}{2}}\right] P_{2 s}(\xi) R_{2 s}(\eta)\right\} .
\end{aligned}
$$

From eq 197, 194, and 187 we find

$$
S_{00}(r)=-2 \pi a_{1} a_{2} \log \left(\frac{r}{A_{1}}\right) \text { when } r>A_{1} .
$$

When $0<r<A_{1}, S_{00}(r)$ is found from eq 187 and 197 by placing $\eta=0$ and $\xi=\xi_{r}=\sqrt{1-\frac{r^{2}}{A_{1}^{2}}}$

$$
\begin{aligned}
S_{00}(r)= & -\frac{a_{1} a_{2}}{2}\left\{4 \pi \log \left(1+\xi_{\tau}\right)\right. \\
& \left.+\frac{2}{\sqrt{\pi}} \sum_{s=0}^{\infty}(-1)^{s} \frac{\Gamma(s+1)}{\Gamma\left(s+\frac{3}{2}\right)}\left[A_{2 s+2}^{00}\left(a_{1}, a_{2}\right)-A_{2 s}^{00}\left(a_{1}, a_{2}\right)\right] P_{2 s+1}\left(\xi_{\tau}\right)\right\}
\end{aligned}
$$

for the range $0<r<A_{1}$. In the part of this range $0<r<A_{2}$ where $\sigma_{00}=0$, this development must represent

as shown by eq 186 .

$$
S_{00}(r)=C_{0}+C_{1} \log r,
$$

If the development eq 200 converges when $r=0\left(\xi_{r}=1\right)$, the constant $C_{1}$ must be zero.

Now by eq 200 , when $r=0, \xi_{r}=1$ and $P_{2 s+1}(1)=1$. Also reference to eq 138 shows that

$$
A_{2 s}^{00}=\frac{\pi \Gamma^{2}\left(s+\frac{1}{2}\right)}{\Gamma^{2}(s+1)} P_{2 s}\left(\xi_{0}^{\prime}\right) .
$$

Hence from eq 200 we find

where

$$
S_{00}(0)=-2 \pi a_{1} a_{2}\left\{\log 2-F\left(\xi_{0}^{\prime}\right)\right\},
$$

$$
F\left(\xi_{0}^{\prime}\right) \equiv 1+\frac{1}{\sqrt{\pi}} \sum_{s=1}^{\infty}(-1)^{s} \frac{\Gamma\left(s+\frac{1}{2}\right)}{\Gamma(s+1)}\left[\frac{1}{2 s}+\frac{1}{2 s+1}\right] P_{2 s}\left(\xi_{0}^{\prime}\right)
$$

Since this series converges, the constant $C_{1}$ in eq 201 is zero. It is readily found that $F\left(\xi_{0}^{\prime}\right)$ is the development

$$
F\left(\xi_{0}^{\prime}\right)=-\log \left[\frac{1+\left|\xi_{0}^{\prime}\right|}{4}\right]=-\log \frac{A_{1}+A_{2}}{4 A_{1}},
$$


so that

$$
\begin{aligned}
S_{00}(0)=-2 \pi a_{1} a_{2} \log \frac{A_{1}+A_{2}}{2 A_{1}}, \text { where } \frac{A_{1}+A_{2}}{2} & =a_{1} \text { if } a_{2}<a_{1} \\
& =a_{2} \text { if } a_{2}>a_{1} .
\end{aligned}
$$

Hence eq 201 becomes

$$
\left.\begin{array}{rl}
S_{00}(r) & =-2 \pi a_{1} a_{2} \log \left(\frac{a_{1}}{a_{1}+a_{2}}\right) \text { if } a_{2}<a_{1} \\
& =-2 \pi a_{1} a_{2} \log \left(\frac{a_{2}}{a_{1}+a_{2}}\right) \text { if } a_{2}>a_{1}
\end{array}\right\} \text { when } 0 \leqslant r \leqslant A_{2} .
$$

Also for the range $A_{2}<r<A_{1}$, we have

$$
\begin{aligned}
S_{00}(r) & =2 a_{1} a_{2}\left\{2 \psi \log \frac{a_{1}}{r}+\pi \log \left(\frac{a_{1}+a_{2}}{a_{1}}\right)-\sum_{k=1}^{\infty}\left(\frac{a_{2}}{a_{1}}\right)^{k} \frac{\sin 2 k \psi}{k^{2}}\right\} \text { if } a_{2}<a_{1} \\
& =2 a_{1} a_{2}\left\{2 \psi \log \frac{a_{2}}{r}+\pi \log \left(\frac{a_{1}+a_{2}}{a_{2}}\right)-\sum_{k=1}^{\infty}\left(\frac{a_{1}}{a_{2}}\right)^{k} \frac{\sin 2 k \psi}{k^{2}}\right\} \text { if } a_{2}>a_{1}
\end{aligned}
$$

Finally, for the remaining range of $r$,

$$
S_{00}(r)=-2 \pi a_{1} a_{2} \log \left(\frac{r}{a_{1}+a_{2}}\right) \text { when } A_{1}<r<\infty .
$$

This completes the expansions required. Before summarizing the results, we should consider a small correction which takes account of the diameter of the wires.

\section{CORRECTIONS FOR THE DIAMETER OF THE WIRES}

If a helix is made with ordinary wire, a plane section of the wire by a plane through the $x$-axis may be considered circular. Let $\rho_{1}$ and $\rho_{2}$ be the wire radii, and let $a_{1}$ and $a_{2}$ represent the cylindrical radii of the central helical filament of the actual helices. Since $\rho_{1}$ and $\rho_{2}$ cannot be larger than $\pi p_{1}$ or $\pi p_{2}$ the correction terms for finite thickness of the wire will be second-order infinitesimals.

Assume that the current density at any point in the wire is in the direction of the generating helical filament passing through that point, its magnitude being a function of the distance $r$, of the point from the axis of the cylinder on which the wire is wound. Let $\gamma_{1}$ (or $\gamma_{2}$ ) be the value, at the central filament, of the $r$-derivative of the current density.

It may then be shown that the correction for the thickness of the wires may be made by using for the cylindrical radii $a_{1}, a_{2}$ in the principal term not $a_{1}, a_{2}$ of the central filaments of the wires, but $\bar{a}_{1}, \bar{a}_{2}$ of their equivalent helices, where

$$
\bar{a}_{1}=a_{1}+\frac{\rho_{1}^{2}}{8}\left(\frac{1}{a_{1}}+2 \gamma_{1}\right) \text { and } \bar{a}_{2}=a_{2}+\frac{\rho_{2}^{2}}{8}\left(\frac{1}{a_{2}}+2 \gamma_{2}\right) \text {. }
$$

If the current is uniform, $\gamma_{1}=\gamma_{2}=0$. If it is the "natural" distribution in both wires (inversely proportional to $r$ ) then $\gamma_{1}=-1 / a_{1}$ and 
$\gamma_{2}=-1 / a_{2}$, so that the correction is equal in magnitude but opposite in sign to that of the uniform distribution.

The derivation of eq 203 is almost verbatim, the same as that given in the paper on coaxial helices (footnote 2). It depends essentially on the fact that $\phi_{11}$ satisfies the partial differential equation 65 .

\section{SUMMARY}

The mutual inductance, $M$, between two parallel helices is expressed in eq 27 in terms of a function $\omega\left(x, \theta_{1}, \theta_{2}\right)$ involving four sets of terminal $x$-differences defined in eq 22 and $22^{\prime}$, where $\theta_{1}$ and $\theta_{2}$ are the associated terminal azimuths, as given in eq 29 . In eq 33 and 58 $\omega$ is resolved into four others, $\omega_{11}+p_{1} p_{2} \omega_{00} / a_{1} a_{2}+\omega_{1}+\omega_{2}$, the first two, $\omega_{11}+p_{1} p_{2} \omega_{00} / a_{1} a_{2}$, corresponding to the mutual inductance of the two associated current sheets, the principal term $\omega_{11}$ representing the effect of angular components of current, the relatively small term, $p_{1} p_{2} \omega_{00} / a_{1} a_{2}$, of second order representing their axial currents. The terms $\omega_{1}$ and $\omega_{2}$ depend upon $x$ and the terminal azimuths. These are expanded, eq 61 and 62, in Fourier's series of the terminal azimuths, the coefficients involving functions $\omega^{\prime}{ }_{n_{1}, n_{2}}$ and $\phi_{n_{1} n_{2}}$. It is shown that two terms of the series for the first-order quantity $\omega_{1}$ are sufficient in general, the second-order term $\omega_{2}$ being given by retaining terms in the single and double series corresponding to $n_{1}=1,2$ and $n_{2}=1,2$.

In eq 140, 144, and 146 three different types of expansions for any function $\phi_{n_{1} n_{2}}$ in oblate spheroidal harmonics are given. In section VII the first and second partial integrals of these expansions are evaluated, giving the functions $\omega^{\prime}{ }_{n_{1} n_{2}}$ and $\omega_{n_{1}, n_{2}}$ in spheroidal harmonics. The results are found in eq 149 to 202. Finally, in eq 203, is given the effect of diameter of the wires.

Washington, August 8, 1940. 